\title{
Intermolecular C-N Addition of Amides and S-N Addition of Sulfinamides to Arynes
}

\author{
Zhijian Liu and Richard C. Larock* \\ Department of Chemistry, Iowa State University, Ames, Iowa 50011
}

\section{Supporting Information}

General. The ${ }^{1} \mathrm{H},{ }^{19} \mathrm{~F}$ and ${ }^{13} \mathrm{C}$ NMR spectra were recorded at 300 and $75.5 \mathrm{MHz}$ or 400 and $100 \mathrm{MHz}$ respectively. All melting points are uncorrected. High resolution mass spectra were recorded on a Kratos MS50TC double focusing magnetic sector mass spectrometer using EI at $70 \mathrm{eV}$. All reagents were used directly as obtained commercially unless otherwise noted. All yields reported represent an average of at least two independent runs. The silylaryl triflate 1a, CsF and acetonitrile were purchased from Sigma-Aldrich Co. The substituted silylaryl triflates 1b, 1c, 1d and 1e were prepared according to a literature procedure. ${ }^{1}$

Preparation of the starting materials. All the $N$-aryltrifluoroacetamides were either commercially available or easily prepared from commercially available materials.<smiles>O=C(N(c1ccccc1)c1ccccc1I)C(F)(F)F</smiles>

$N$-(2-Iodophenyl), $N$-phenyltrifluoroacetamide. To a solution of $1.48 \mathrm{~g}$ of of $\mathrm{N}$ phenyl-2-iodoaniline ${ }^{2}(5 \mathrm{mmol})$ and $1.01 \mathrm{~g}$ of triethylamine $(10 \mathrm{mmol})$ in $15 \mathrm{~mL}$ of $\mathrm{CH}_{2} \mathrm{Cl}_{2}$ at $0{ }^{\circ} \mathrm{C}$ was added $2.1 \mathrm{~g}$ of trifluoroacetic anhydride $(10 \mathrm{mmol})$. The reaction mixture was stirred at room temperature of $1 \mathrm{~d}$. The resulting solution was washed with brine $(20 \mathrm{~mL})$ and extracted with $\mathrm{CH}_{2} \mathrm{Cl}_{2}(20 \mathrm{~mL})$. The combined $\mathrm{CH}_{2} \mathrm{Cl}_{2}$ fractions were dried over $\mathrm{Na}_{2} \mathrm{SO}_{4}$ and concentrated under reduced pressure. The residue was purified by flash chromatography on silica gel to afford $1.66 \mathrm{~g}$ of the desired product (85\% yield) as 
a white solid: mp 93-94 ${ }^{\circ} \mathrm{C} ;{ }^{1} \mathrm{H}$ NMR (300 MHz, $\left.\mathrm{CDCl}_{3}\right) \delta$ 7.00-7.62 (m, 8H), 7.94 (d, $J$ $=7.8 \mathrm{~Hz}, 1 \mathrm{H}) ;{ }^{19} \mathrm{~F}$ NMR: -68.0; IR $\left(\mathrm{CDCl}_{3}, \mathrm{~cm}^{-1}\right)$ 3064, 1711, 1593; Anal Calcd. for $\mathrm{C}_{12} \mathrm{H}_{9} \mathrm{~F}_{3} \mathrm{INO}$ : C, 42.99; H, 2.32; N, 3.58. Found: C, 42.97; H, 2.58; N, 3.65; HRMS m/z 391.9687 (calcd $\mathrm{C}_{12} \mathrm{H}_{9} \mathrm{~F}_{3} \mathrm{INO}, 391.9680$ ).

All of the $\mathrm{N}$-aryltrifluoromethanesulfinamides have been prepared by the following procedure. To a solution of $1.56 \mathrm{~g}(10 \mathrm{mmol})$ of sodium trifluoromethanesulfinate (purity $97 \%$ ) in $10 \mathrm{~mL}$ of ethyl acetate was added at room temperature $0.467 \mathrm{~mL}$ of phosphoryl chloride. The resulting mixture was stirred for $5 \mathrm{~min}$, then $5 \mathrm{mmol}$ of aryl amine was added dropwise and the reaction mixture was stirred for an additional $30 \mathrm{~min}$. The reaction mixture was then washed with brine (20 mL) and extracted with ethyl acetate $(20 \mathrm{~mL})$. The combined ethyl acetate fractions were dried over $\mathrm{Na}_{2} \mathrm{SO}_{4}$ and concentrated under reduced pressure. The residue was purified by flash chromatography on silica gel to afford the desired product.<smiles>O=S(Nc1ccccc1)C(F)(F)F</smiles>

$N$-Phenyltrifluoromethanesulfinamide. The indicated compound was obtained in an 82\% yield as a white solid: mp 63-64 ${ }^{\circ} \mathrm{C}$ (lit. ${ }^{3} \mathrm{mp} 64{ }^{\circ} \mathrm{C}$ ). The ${ }^{1} \mathrm{H},{ }^{19} \mathrm{~F}$ and ${ }^{13} \mathrm{C}$ NMR spectra match the literature data. ${ }^{3}{ }^{1} \mathrm{H}$ NMR (300 MHz, $\left.\mathrm{CDCl}_{3}\right) \delta 6.81$ (s, 1H), 7.09 (d, $J$ = 7.8, 2H), 7.18 (t, $J=7.5 \mathrm{~Hz}, 1 \mathrm{H}), 7.34$ (t, $J=7.8 \mathrm{~Hz}, 2 \mathrm{H}) ;{ }^{13} \mathrm{C}$ NMR $\left(75 \mathrm{MHz}, \mathrm{CDCl}_{3}\right)$ $\delta$ 138.1, 129.9, 125.4, 123.7, 123.7 (q, $J=333.4 \mathrm{~Hz}$ ), 120.3; ${ }^{19}$ F NMR: -78.1.<smiles>O=S(Nc1ccc(I)cc1)C(F)(F)F</smiles>

$N$-(4-Iodophenyl)trifluoromethanesulfinamide. The indicated compound was obtained in an 86\% yield as a white solid: mp 94-95 ${ }^{\circ} \mathrm{C} ;{ }^{1} \mathrm{H}$ NMR (400 $\left.\mathrm{MHz} \mathrm{CDCl}_{3}\right) \delta$ $6.83(\mathrm{td}, J=8.8,2.8 \mathrm{~Hz}, 2 \mathrm{H}), 7.24$ (s, $1 \mathrm{H}), 7.61$ (td, $J=8.8,2.8 \mathrm{~Hz}, 2 \mathrm{H}) ;{ }^{13} \mathrm{C}$ NMR $(100$ $\mathrm{MHz} \mathrm{CDCl}_{3}$ ) $\delta 138.9,138.4,123.7$ (q, $J=331.4 \mathrm{~Hz}$ ), 121.8, 88.9; ${ }^{19} \mathrm{~F}$ NMR: -77.7; IR $\left(\mathrm{CDCl}_{3}, \mathrm{~cm}^{-1}\right)$ 3210, 3172, 3023, 2903, 1587, 1487, 1188; HRMS m/z 335.9142 (calcd $\mathrm{C}_{7} \mathrm{H}_{5} \mathrm{~F}_{3} \mathrm{INOS}$, 335.9136). 
<smiles>COc1ccc(NS(=O)C(F)(F)F)cc1</smiles>

$N$-(4-Methoxyphenyl)trifluoromethanesulfinamide. The indicated compound was obtained in an 84\% yield as a white solid: mp 84-85 ${ }^{\circ} \mathrm{C} ;{ }^{1} \mathrm{H}$ NMR $\left(300 \mathrm{MHz}, \mathrm{CDCl}_{3}\right) \delta$ 3.79 (s, 3H), 6.61 (s, 1H), 6.86 (td, $J=9.0,2.4 \mathrm{~Hz}, 2 \mathrm{H}), 7.08$ (td, $J=9.0,2.4 \mathrm{~Hz}, 2 \mathrm{H})$; ${ }^{13} \mathrm{C} \mathrm{NMR}\left(75 \mathrm{MHz}, \mathrm{CDCl}_{3}\right) \delta 158.3,130.6,124.4,123.8$ (q, $\left.J=331.9 \mathrm{~Hz}\right), 115.1 ;{ }^{19} \mathrm{~F}$ NMR: -78.1; IR $\left(\mathrm{CDCl}_{3}, \mathrm{~cm}^{-1}\right)$ 3174, 2966, 2916, 2846, 1511, 1183; HRMS m/z 239.0231(calcd $\mathrm{C}_{8} \mathrm{H}_{8} \mathrm{~F}_{3} \mathrm{NO}_{2} \mathrm{~S}$ 239.0227).

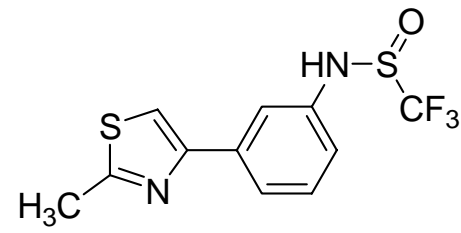

$N$-[3-(2-Methyl-4-thiazolyl)phenyl]trifluoromethanesulfinamide. The indicated compound was obtained in an 80\% yield as a white solid: mp 73-74 ${ }^{\circ} \mathrm{C}$; ${ }^{1} \mathrm{H}$ NMR (300 $\left.\mathrm{MHz} \mathrm{CDCl}_{3}\right) \delta 2.67$ (s, 3H), 6.98 (dd, $\left.J=7.8,2.1 \mathrm{~Hz}, 1 \mathrm{H}\right), 7.21-7.24$ (m, 2H), 7.54 (d, $J$ $=7.8 \mathrm{~Hz}, 1 \mathrm{H}), 7.63(\mathrm{t}, J=1.8 \mathrm{~Hz}, 1 \mathrm{H}), 8.16(\mathrm{~s}, 1 \mathrm{H}) ;{ }^{13} \mathrm{C} \mathrm{NMR}\left(75 \mathrm{MHz}, \mathrm{CDCl}_{3}\right) \delta 166.7$, 154.1, 139.4, 136.1, 130.3, 124.1 (q, $J=331.9 \mathrm{~Hz}), 122.9,119.4,118.2,113.6,19.2 ;{ }^{19} \mathrm{~F}$ NMR: -77.4; IR $\left(\mathrm{CDCl}_{3}, \mathrm{~cm}^{-1}\right)$ 3207, 3113, 2926, 2828, 2759, 1607, 1587; HRMS m/z 306.0114 (calcd $\mathrm{C}_{11} \mathrm{H}_{9} \mathrm{~F}_{3} \mathrm{~N}_{2} \mathrm{OS}_{2}, 306.0108$ ).<smiles>O=S(Nc1ccc(Br)cc1)C(F)(F)F</smiles>

$N$-(4-Bromophenyl)trifluoromethanesulfinamide. The indicated compound was obtained in an 89\% yield as a white solid: mp 96-97 ${ }^{\circ} \mathrm{C} ;{ }^{1} \mathrm{H}$ NMR $\left(300 \mathrm{MHz}, \mathrm{CDCl}_{3}\right) \delta$ 6.97 (td, $J=8.8,2.8 \mathrm{~Hz}, 2 \mathrm{H}), 7.02$ (s, $1 \mathrm{H}), 7.44$ (td, $J=8.8,2.8 \mathrm{~Hz}, 2 \mathrm{H}) ;{ }^{13} \mathrm{C}$ NMR $(75$ $\left.\mathrm{MHz} \mathrm{CDCl}_{3}\right) \delta 137.5,133.0,123.7$ (q, $J=331.6 \mathrm{~Hz}$ ), 121.9, 118.6; ${ }^{19} \mathrm{~F}$ NMR: -77.8; IR $\left(\mathrm{CDCl}_{3}, \mathrm{~cm}^{-1}\right)$ 3214, 3030, 2914, 1490, 1208; HRMS m/z 289.9231 (calcd $\mathrm{C}_{7} \mathrm{H}_{5} \mathrm{BrF}_{3} \mathrm{NOS}$, 286.9227). 
<smiles>O=S(Nc1ccc(Cl)cc1)C(F)(F)F</smiles>

$N$-(4-Chlorophenyl)trifluoromethanesulfinamide. The indicated compound was obtained in a $91 \%$ yield as a white solid: $\mathrm{mp} 89-90{ }^{\circ} \mathrm{C} ;{ }^{1} \mathrm{H}$ NMR $\left(300 \mathrm{MHz}, \mathrm{CDCl}_{3}\right) \delta$ 7.02 (td, $J=8.8,2.8 \mathrm{~Hz}, 2 \mathrm{H}), 7.15$ (s, $1 \mathrm{H}), 7.28$ (td, $J=8.8,2.8 \mathrm{~Hz}, 2 \mathrm{H}) ;{ }^{13} \mathrm{C}$ NMR $(75$ $\left.\mathrm{MHz}, \mathrm{CDCl}_{3}\right) \delta 137.0,131.1,130.0,123.8$ (q, $\left.J=331.8 \mathrm{~Hz}\right), 121.7 ;{ }^{19} \mathrm{~F}$ NMR: -77.8; IR $\left(\mathrm{CDCl}_{3}, \mathrm{~cm}^{-1}\right)$ 3213, 2916, 2848, 1494, 1209; HRMS m/z 242.9737 (calcd $\left.\mathrm{C}_{7} \mathrm{H}_{5} \mathrm{ClF}_{3} \mathrm{NOS}, 242.9732\right)$.<smiles>O=C(Nc1ccc(NS(=O)C(F)(F)F)cc1)C(F)(F)F</smiles>

\section{$N$-[4-(Trifluoroacetamido)phenyl]-2,2,2-trifluoromethanesulfinamide. The} indicated compound was obtained in a $61 \%$ yield as a white solid: mp $160-162{ }^{\circ} \mathrm{C} ;{ }^{1} \mathrm{H}$ NMR (300 MHz, acetone- $\mathrm{d}_{6}$ ) $\delta 7.30$ (dt, $\left.J=9.0,2.8 \mathrm{~Hz}, 2 \mathrm{H}\right), 7.75$ (dt, $J=9.0,2.8 \mathrm{~Hz}$, 2H), 9.01 (s, 1H), 10.30 (s, 1H); ${ }^{13} \mathrm{C}$ NMR (100 MHz, acetone-d $\left.\mathrm{d}_{6}\right) \delta 155.0$ (q, $J=35.2$ $\mathrm{Hz}$ ), 124.3 (q, $J=333.0 \mathrm{~Hz}$ ), 122.4, 120.7, 116.2 (q, $J=286.0 \mathrm{~Hz}$ ); ${ }^{19} \mathrm{~F}$ NMR: -76.5, 78.4; IR $\left(\mathrm{CDCl}_{3}, \mathrm{~cm}^{-1}\right)$ 3288, 3220, 3094, 1695, 1547; HRMS m/z 320.0058 (calcd $\left.\mathrm{C}_{9} \mathrm{H}_{6} \mathrm{lF}_{6} \mathrm{~N}_{2} \mathrm{O}_{2} \mathrm{~S}, 320.0059\right)$.<smiles>O=S(NCc1ccccc1)C(F)(F)F</smiles>

$N$-Benzyltrifluoromethanesulfinamide The indicated compound was obtained in a 92\% yield as white solid: mp 26-27 ${ }^{\circ} \mathrm{C} ;{ }^{1} \mathrm{H}$ NMR (300 MHz, $\mathrm{CDCl}_{3}$ ) $\delta$ 4.27-4.34 (m, 1H), 4.43-4.50 (m, 1H), 4.69 (s, 1H), 7.29-7.40 (m, 7H); $\left.{ }^{13} \mathrm{C} \mathrm{NMR} \mathrm{(75} \mathrm{MHz,} \mathrm{CDCl}_{3}\right) \delta 136.7$, 129.2, 128.5, 128.3, 123.9 (q, $J=331.8 \mathrm{~Hz}), 46.6$ (q, $J=0.9 \mathrm{~Hz}$ ); ${ }^{19}$ F NMR: -77.1; IR $\left(\mathrm{CDCl}_{3}, \mathrm{~cm}^{-1}\right)$ 3234, 3093, 3071, 3037, 2881, 1457; HRMS m/z 233.0237 (calcd $\left.\mathrm{C}_{7} \mathrm{H}_{5} \mathrm{ClF}_{3} \mathrm{NOS}, 233.02333\right)$.

\section{General procedure for the intermolecular $\mathrm{C}-\mathrm{N}$ addition of amides to arynes.}

To a solution of the $N$-aryltrifluoroacetamide $(0.5 \mathrm{mmol})$ and silylaryl triflate $(0.6$ $\mathrm{mmol})$ in acetonitrile $(6 \mathrm{~mL})$ was added CsF $(1.0 \mathrm{mmol})$. The solution was allowed to stir at room temperature for $4 \mathrm{~h}$. The resulting solution was washed with brine $(20 \mathrm{~mL})$ 
and extracted with diethyl ether $(20 \mathrm{~mL})$. The combined ether fractions were dried over $\mathrm{Na}_{2} \mathrm{SO}_{4}$ and concentrated under reduced pressure. The residue was purified by flash chromatography on silica gel to afford the desired product.<smiles>O=C(c1ccccc1Nc1ccccc1)C(F)(F)F</smiles>

1-[2-(Phenylamino)phenyl]trifluoroethanone. The indicated compound was obtained in a 77\% yield (102 mg) as a yellow solid: mp 70-72 ${ }^{\circ} \mathrm{C}$; ${ }^{1} \mathrm{H}$ NMR $(300 \mathrm{MHz}$, $\left.\mathrm{CDCl}_{3}\right) \delta 6.76$ (dt, $\left.J=7.8,1.2 \mathrm{~Hz}, 1 \mathrm{H}\right), 7.18-7.27$ (m, 4H), 7.35-7.42 (m, 3H), 7.83-7.87 (m, 1H), 10.24 (s, $\left.1 \mathrm{H}) ;{ }^{13} \mathrm{C} \mathrm{NMR} \mathrm{(75} \mathrm{MHz,} \mathrm{CDCl}_{3}\right) \delta 181.2$ (q, $\left.J=33.2 \mathrm{~Hz}\right), 151.5$, 139.1, 137.1, 132.2 (q, $J=4.1 \mathrm{~Hz}), 129.9,125.8,124.6,117.4$ (q, $J=289.6 \mathrm{~Hz}), 117.1$, 114.7, 112.1; ${ }^{19}$ F NMR: -69.4; IR $\left(\mathrm{CDCl}_{3}, \mathrm{~cm}^{-1}\right)$ 3306, 3088, 3040, 1658. HRMS m/z 265.0720 (calcd $\mathrm{C}_{14} \mathrm{H}_{10} \mathrm{~F}_{3} \mathrm{NO}, 265.0714$ ).<smiles>O=C(c1ccccc1Nc1ccc(Cl)cc1)C(F)(F)F</smiles>

1-[2-(4-Chlorophenylamino)phenyl]trifluoroethanone. The indicated compound was obtained in an $86 \%$ yield $\left(128 \mathrm{mg}\right.$ ) as a yellow solid: mp $76-77^{\circ} \mathrm{C}$; ${ }^{1} \mathrm{H}$ NMR (300 $\left.\mathrm{MHz}, \mathrm{CDCl}_{3}\right) \delta 6.79$ (dt, $\left.J=7.8,1.2 \mathrm{~Hz}, 1 \mathrm{H}\right), 7.13-7.25$ (m, 3H), 7.33-7.44 (m, 3H), 7.84-7.88 (m, 1H), 10.15 (s, $1 \mathrm{H}) ;{ }^{13} \mathrm{C}$ NMR $\left(75 \mathrm{MHz}, \mathrm{CDCl}_{3}\right) \delta 181.4$ (q, $J=33.3 \mathrm{~Hz}$ ), 151.1, 137.7, 137.2, 132.3 (q, $J=4.0 \mathrm{~Hz}$ ), 130.9, 129.9, 125.8, 117.6, 117.3 (q, $J=289.5$ $\mathrm{Hz})$, 114.6, 112.4; ${ }^{19} \mathrm{~F}$ NMR: -69.5; IR $\left(\mathrm{CDCl}_{3}, \mathrm{~cm}^{-1}\right)$ 3295, 3089, 1650, 1568; HRMS m/z 299.0329 (calcd $\mathrm{C}_{14} \mathrm{H}_{9} \mathrm{ClF}_{3} \mathrm{NO}$, 299.0329).<smiles>COc1ccc(Nc2ccccc2C(=O)C(F)(F)F)cc1</smiles>

1-[2-(4-Methoxylphenylamino)phenyl]trifluoroethanone. The indicated compound was obtained in a 78\% yield (115 mg) as a yellow solid: mp 77-78 ${ }^{\circ} \mathrm{C} ;{ }^{1} \mathrm{H}$ NMR (300 $\left.\mathrm{MHz}, \mathrm{CDCl}_{3}\right) \delta 3.84(\mathrm{~s}, 3 \mathrm{H}), 6.72(\mathrm{t}, J=7.8 \mathrm{~Hz}, 1 \mathrm{H}), 6.92-7.00$ (m, 3H), 7.16-7.20 (m, 2H), 7.35 (t, $J=7.8 \mathrm{~Hz}, 1 \mathrm{H}), 7.81-7.85$ (m, $1 \mathrm{H}), 10.14$ (s, $1 \mathrm{H}) ;{ }^{13} \mathrm{C}$ NMR $(75 \mathrm{MHz}$, 
$\left.\mathrm{CDCl}_{3}\right) \delta 181.0(\mathrm{q}, J=33.1 \mathrm{~Hz}), 158.0,152.8,137.1,132.0(\mathrm{q}, J=4.0 \mathrm{~Hz}), 131.6,127.1$, 117.5 (q, $J=289.5 \mathrm{~Hz}), 116.5,115.1,114.4,111.4,55.7 ;{ }^{19} \mathrm{~F}$ NMR: -69.4 IR $\left(\mathrm{CDCl}_{3}\right.$, $\mathrm{cm}^{-1}$ ) 3307, 3071, 3045, 2959, 2913, 2843, 1656, 1139. HRMS m/z 295.0823 (calcd $\mathrm{C}_{15} \mathrm{H}_{12} \mathrm{~F}_{3} \mathrm{NO}_{2}, 295.0820$ ).<smiles>CC(=O)c1ccc(Nc2ccccc2C(=O)C(F)(F)F)cc1</smiles>

Methyl 4-[2-trifluoroacetylphenylamino]benzoate. The indicated compound was obtained in a $60 \%$ yield (97 mg) as a yellow solid: mp 82-83 ${ }^{\circ} \mathrm{C}$; ${ }^{1} \mathrm{H}$ NMR (300 MHz, $\left.\mathrm{CDCl}_{3}\right) \delta 3.92(\mathrm{~s}, 3 \mathrm{H}), 6.72(\mathrm{td}, J=7.2,1.5 \mathrm{~Hz}, 1 \mathrm{H}), 7.3(\mathrm{dt}, J=8.7,1.8 \mathrm{~Hz}, 2 \mathrm{H}), 7.41-$ 7.52 (m, 2H), 7.88-7.92 (m, 1H), 8.05 (dt, $J=8.7,1.5 \mathrm{~Hz}, 2 \mathrm{H}), 10.26$ (s, $1 \mathrm{H}) ;{ }^{13} \mathrm{C}$ NMR $\left(75 \mathrm{MHz}, \mathrm{CDCl}_{3}\right) \delta 181.7$ (q, $\left.J=33.6 \mathrm{~Hz}\right), 166.6,149.2,143.9,137.2,132.4$ (q, $J=4.1$ Hz), 131.5, 126.0, 121.6, 118.7, 117.1 (q, $J=289.5 \mathrm{~Hz}), 115.6,113.6,52.3 ;{ }^{19} \mathrm{~F}$ NMR: 69.6; IR $\left(\mathrm{CDCl}_{3}, \mathrm{~cm}^{-1}\right)$ 3308, 2999, 2952, 2844, 1721, 1669, 1592; HRMS m/z 323.0774 (calcd $\mathrm{C}_{16} \mathrm{H}_{12} \mathrm{~F}_{3} \mathrm{NO}_{3}, 323.0769$ ).<smiles>Cc1cc(C)c(Nc2ccccc2C(=O)C(F)(F)F)c(C)c1</smiles>

1-[2-(2,4,6-Trimethylphenylamino)phenyl]trifluoroethanone. The indicated compound was obtained in a 69\% yield (106 mg) as a yellow solid: mp 96-97 ${ }^{\circ} \mathrm{C} ;{ }^{1} \mathrm{H}$ NMR (300 MHz, $\left.\mathrm{CDCl}_{3}\right) \delta 2.17$ (s, 6H), 2.36 (s, 3H), 6.37 (d, $\left.J=8.7 \mathrm{~Hz}, 1 \mathrm{H}\right), 6.71$ (td, $J$ = 7.8, 0.6 Hz, 1H), 7.01 (s, 2H), 7.32 (td, $J=7.8,0.6 \mathrm{~Hz}, 1 \mathrm{H}), 7.86-7.90$ (m, 1H), 9.88 (s, $1 \mathrm{H}) ;{ }^{13} \mathrm{C}$ NMR $\left(75 \mathrm{MHz}, \mathrm{CDCl}_{3}\right) \delta 181.2$ (q, $\left.J=32.9 \mathrm{~Hz}\right), 153.3,137.4,137.3,136.4$, 133.2, 132.1 (q, $J=4.1 \mathrm{~Hz}), 129.6,119.5,117.5$ (q, $J=289.5 \mathrm{~Hz}), 113.9,110.9,21.2$, 18.3; ${ }^{19}$ F NMR: -69.3; IR $\left(\mathrm{CDCl}_{3}, \mathrm{~cm}^{-1}\right)$ 3307, 3015, 2944, 2915, 2856, 1647, 1568; HRMS m/z 307.1188 (calcd $\mathrm{C}_{17} \mathrm{H}_{16} \mathrm{~F}_{3} \mathrm{NO}, 307.1184$ ). 
<smiles>COc1cccc(Nc2ccc(I)cc2)c1C(=O)C(F)(F)F</smiles>

1-[2-(4-Iodophenylamino)-6-methoxyphenyl]trifluoroethanone. The indicated compound was obtained in an $88 \%$ yield (185 mg) as a yellow solid: mp $99-100{ }^{\circ} \mathrm{C} ;{ }^{1} \mathrm{H}$ NMR (300 MHz, $\left.\mathrm{CDCl}_{3}\right) \delta 3.87$ (s, 3H), 6.35 (d, $\left.J=8.1 \mathrm{~Hz}, 1 \mathrm{H}\right), 6.80$ (d, $J=8.4 \mathrm{~Hz}$, 1H), 6.95 (dt, $J=8.7,2.7 \mathrm{~Hz}, 2 \mathrm{H}), 7.29$ (t, $J=8.4 \mathrm{~Hz}, 1 \mathrm{H}), 7.61$ (dt, $J=8.7,2.7 \mathrm{~Hz}, 2 \mathrm{H})$, 8.85 (s, 1H); ${ }^{13} \mathrm{C}$ NMR (75 MHz, $\left.\mathrm{CDCl}_{3}\right) \delta 184.6$ (q, $\left.J=36.4 \mathrm{~Hz}\right), 161.9,148.2,140.0$, 138.6, 136.6, 124.4, 116.8 (q, $J=283.4 \mathrm{~Hz}$ ), 108.8, 108.2, 101.4, 87.3, 56.0; ${ }^{19} \mathrm{~F}$ NMR: 74.1; IR ( $\left.\mathrm{CDCl}_{3}, \mathrm{~cm}^{-1}\right)$ 3360, 3015, 2978, 2945, 2841, 1668, 1579; HRMS m/z 420.9791 (calcd $\mathrm{C}_{15} \mathrm{H}_{11} \mathrm{~F}_{3} \mathrm{INO}_{2}, 420.9786$ ).<smiles>COc1cccc(Nc2cc(Cl)ccc2Cl)c1C(=O)C(F)(F)F</smiles>

\section{1-[2-(2,5-Dichlorophenylamino)-6-methoxylphenyl]trifluoroethanone. The} indicated compound was obtained in an 87\% yield (158 mg) as a yellow solid: mp 95-96 ${ }^{\circ} \mathrm{C} ;{ }^{1} \mathrm{H}$ NMR (300 MHz, $\left.\mathrm{CDCl}_{3}\right) \delta 3.91$ (s, 3H), 6.52 (d, $\left.J=8.1 \mathrm{~Hz}, 1 \mathrm{H}\right), 6.92-6.97$ (m, 2H), 7.33 (d, $J=8.4 \mathrm{~Hz}, 1 \mathrm{H}), 7.38-7.44(\mathrm{~m}, 2 \mathrm{H}), 8.71$ (s, $1 \mathrm{H}) ;{ }^{13} \mathrm{C}$ NMR $(75 \mathrm{MHz}$, $\left.\mathrm{CDCl}_{3}\right) \delta 184.9$ (q, $\left.J=36.9 \mathrm{~Hz}\right), 161.6,145.5,138.8,136.3,133.2,131.1,123.8,123.6$, 120.1, 116.5 (q, $J=288.0 \mathrm{~Hz}), 109.9,109.6,103.3$, 56.1; ${ }^{19} \mathrm{~F}$ NMR: -74.1; IR $\left(\mathrm{CDCl}_{3}\right.$, $\mathrm{cm}^{-1}$ ) 3364, 3053, 2985, 2919, 2856, 1580, 1474; HRMS m/z 363.0198 (calcd $\left.\mathrm{C}_{15} \mathrm{H}_{10} \mathrm{Cl}_{2} \mathrm{~F}_{3} \mathrm{NO}_{2}, 363.0194\right)$.<smiles>COc1cc(Nc2ccc(C)cc2)c(C(=O)C(F)(F)F)cc1OC</smiles>

1-[2-(4-Methylphenylamino)-4,5-dimethoxyphenyl]trifluoroethanone. The indicated compound was obtained in a 70\% yield (118 mg) as a yellow solid: mp 105-106 ${ }^{\circ} \mathrm{C} ;{ }^{1} \mathrm{H}$ NMR (300 MHz, $\left.\mathrm{CDCl}_{3}\right) \delta 2.37$ (s, 3H), 3.77 (s, 3H), 3.85 (s, 3H), 6.59 (s, 1H), 7.14-7.23 (m, 5H), 10.51 (s, 1H); ${ }^{13} \mathrm{C}$ NMR (75 MHz, $\left.\mathrm{CDCl}_{3}\right) \delta 178.0(\mathrm{q}, J=32.5 \mathrm{~Hz}$ ), 
158.1, 150.6, 141.1, 136.6, 135.5, 130.5, 124.6, 117.8 (q, $J=289.3 \mathrm{~Hz}), 116.6$ (q, $J=3.5$ $\mathrm{Hz})$, 104.4, 95.9, 56.5, 56.1, 21.2; ${ }^{19} \mathrm{~F}$ NMR: -69.5; IR $\left(\mathrm{CDCl}_{3}, \mathrm{~cm}^{-1}\right)$ 3260, 2997, 2969, 2939, 2836, 1633, 1517; HRMS m/z 339.1086 (calcd $\mathrm{C}_{17} \mathrm{H}_{17} \mathrm{~F}_{3} \mathrm{NO}_{3}$, 339.1082).<smiles>Cc1ccc(Nc2cc(C)c(C)cc2C(=O)C(F)(F)F)cc1</smiles>

1-[2-(4-Methylphenylamino)-4,5-dimethylphenyl]trifluoroethanone. The indicated compound was obtained in a $58 \%$ yield (89 mg) as a yellow solid: mp $85-86{ }^{\circ} \mathrm{C} ;{ }^{1} \mathrm{H}$ NMR (300 MHz, CDCl $\left.{ }_{3}\right) \delta 2.18$ (s, 3H), 2.19 (s, 3H), 2.37 (s, 3H), 6.95 (s, 1H), 7.13 (d, $J=8.4,2 \mathrm{H}), 7.20$ (d, $J=8.4,2 \mathrm{H}), 7.53$ (d, $J=1.8,1 \mathrm{H}), 10.09$ (s, $1 \mathrm{H}) ;{ }^{13} \mathrm{C}$ NMR $(100$ $\left.\mathrm{MHz}, \mathrm{CDCl}_{3}\right) \delta 180.2$ (q, $J=32.8 \mathrm{~Hz}$ ), 150.6, 148.3, 136.7, 135.2, 131.5 (q, $J=3.9 \mathrm{~Hz}$ ), 130.4, 135.6, 124.7, 117.6 (q, $J=289.2 \mathrm{~Hz}$ ), 114.9, 110.2, 21.2, 19.2; ${ }^{19}$ F NMR: -69.3; IR $\left(\mathrm{CDCl}_{3}, \mathrm{~cm}^{-1}\right)$ 3287, 2943, 2922, 1646, 1516; HRMS m/z 307.1188 (calcd $\left.\mathrm{C}_{17} \mathrm{H}_{16} \mathrm{~F}_{3} \mathrm{NO}, 307.1184\right)$.<smiles>COc1cccc(Nc2ccc(C)cc2)c1C(=O)C(F)(F)Cl</smiles>

\section{1-[2-(4-Methylphenylamino)-6-methoxyphenyl]-2 -chloro-2 ,2'-difluoroethanone.}

The indicated compound was obtained in a $66 \%$ yield $(107 \mathrm{mg})$ as a yellow solid: $\mathrm{mp} 72-$ $73{ }^{\circ} \mathrm{C}$; ${ }^{1} \mathrm{H}$ NMR (300 MHz, $\left.\mathrm{CDCl}_{3}\right) \delta 2.33$ (s, 3H), 3.85 (s, 3H), $6.28(\mathrm{~d}, J=8.1 \mathrm{~Hz}, 1 \mathrm{H})$, 6.74 (d, $J=8.4 \mathrm{~Hz}, 1 \mathrm{H}), 7.07$ (d, $J=8.4 \mathrm{~Hz}, 2 \mathrm{H}), 7.14$ (d, $J=8.4 \mathrm{~Hz}, 2 \mathrm{H}), 7.23$ (t, $J=$ $8.4 \mathrm{~Hz}, 1 \mathrm{H}), 8.55$ (s, $1 \mathrm{H}) ;{ }^{13} \mathrm{C} \mathrm{NMR}\left(75 \mathrm{MHz}, \mathrm{CDCl}_{3}\right) \delta 186.3(\mathrm{t}, J=30.4 \mathrm{~Hz}), 161.4$, 149.3, 137.5, 136.0, 134.4, 130.2, 123.3, 121.0 (t, $J=320.7 \mathrm{~Hz}$ ), 108.1, 107.7, 100.3, 55.6, 21.1; ${ }^{19}$ F NMR: -62.2; IR $\left(\mathrm{CDCl}_{3}, \mathrm{~cm}^{-1}\right)$ 3369, 3028, 2942, 2842, 1657; HRMS m/z 326.0762 (calcd $\mathrm{C}_{16} \mathrm{H}_{14} \mathrm{ClF}_{2} \mathrm{NO}_{2}, 326.0759$ ). 
<smiles>COc1ccc(Nc2ccccc2)c(C(=O)C(F)(F)F)c1</smiles>

1-(2-phenylamino-5-methoxyphenyl)trifluoroethanone. The indicated compound was obtained in a 35\% yield (51 mg) as a yellow solid: mp 77-79 ${ }^{\circ} \mathrm{C} ;{ }^{1} \mathrm{H}$ NMR (300 $\left.\mathrm{MHz} \mathrm{CDCl}_{3}\right) \delta 3.79$ (s, 3H), 7.08-7.22 (m, 6H), 7.37 (td, $\left.J=8.1,1.8,2 \mathrm{H}\right), 10.03$ (s. 1H); ${ }^{13} \mathrm{C}$ NMR (75 MHz, $\left.\mathrm{CDCl}_{3}\right) \delta 180.5$ (q, $\left.J=33.3 \mathrm{~Hz}\right), 150.7,146.6,139.7,129.8,127.3$, 125.1, 123.8, 117.4 (q, $J=289.6 \mathrm{~Hz}), 116.7,112.4$ (q, $J=4.3 \mathrm{~Hz}), 111.9,55.9 ;{ }^{19} \mathrm{~F}$ NMR: -69.7; IR $\left(\mathrm{CDCl}_{3}, \mathrm{~cm}^{-1}\right)$ 3310, 3002, 2933, 2855, 1657, 1520; HRMS m/z 295.0823 (calcd $\mathrm{C}_{15} \mathrm{H}_{12} \mathrm{~F}_{3} \mathrm{NO}_{2}, 295.0820$ ).<smiles>COc1ccc(C(=O)C(F)(F)F)c(Nc2ccccc2)c1</smiles>

1-(2-phenylamino-4-methoxyphenyl)trifluoroethanone. The indicated compound was obtained in a 38\% yield (56 mg) as a yellow solid: mp 80-82 ${ }^{\circ} \mathrm{C} ;{ }^{1} \mathrm{H}$ NMR (300 $\left.\mathrm{MHz}^{\mathrm{CDCl}}{ }_{3}\right) \delta 3.75$ (s, 3H), 6.34 (dd, $J=9.3,2.7 \mathrm{~Hz}, 1 \mathrm{H}$ ), 6.57 (d, $J=2.4 \mathrm{~Hz}, 1 \mathrm{H}$ ), 7.20-7.30 (m, 3H), 7.41 (td, $J=8.1,1.8,2 \mathrm{H}$ ), 7.77 (dd, $J=9.0,2.1 \mathrm{~Hz}, 1 \mathrm{H}), 10.48$ (s. $1 \mathrm{H}) ;{ }^{13} \mathrm{C}$ NMR $\left(75 \mathrm{MHz}, \mathrm{CDCl}_{3}\right) \delta 179.2$ (q, $\left.J=32.8 \mathrm{~Hz}\right), 166.7,154.3,139.1,134.4$ (q, $J=4.3 \mathrm{~Hz}$ ), 129.9, 125.8, 124.8, 117.6 (q, $J=289.4 \mathrm{~Hz}), 106.7,106.6,96.4,55.6 ;{ }^{19} \mathrm{~F}$ NMR: -69.22; IR $\left(\mathrm{CDCl}_{3}, \mathrm{~cm}^{-1}\right)$ 3288, 3024, 2967, 2940, 1643, 1618; HRMS m/z 295.0823 (calcd $\mathrm{C}_{15} \mathrm{H}_{12} \mathrm{~F}_{3} \mathrm{NO}_{2}, 295.0820$ ).<smiles>O=C(c1ccccc1Nc1ccc(Nc2ccccc2C(=O)C(F)(F)F)cc1)C(F)(F)F</smiles>

1,1'-[1,4-Phenylenebis(imino-2,1-phenylene)]bistrifluoroethanone. The indicated compound was obtained in a $48 \%$ yield (109 mg) as a yellow solid: mp $186-188{ }^{\circ} \mathrm{C} ;{ }^{1} \mathrm{H}$ NMR (300 MHz, $\left.\mathrm{CDCl}_{3}\right) \delta 6.80$ (t, $\left.J=7.5 \mathrm{~Hz}, 2 \mathrm{H}\right), 7.23$ (d, $\left.J=8.7 \mathrm{~Hz}, 2 \mathrm{H}\right), 7.31$ (s, 4H), 7.43 (t, $J=7.2 \mathrm{~Hz}, 2 \mathrm{H}), 7.86-7.89$ (m, 2H), 10.23 (s, 1H); ${ }^{13} \mathrm{C}$ NMR (75 MHz, $\left.\mathrm{CDCl}_{3}\right) \delta 181.3(\mathrm{q}, J=33.4 \mathrm{~Hz}), 161.4,137.2,136.4,132.3(\mathrm{q}, J=4.2 \mathrm{~Hz}), 125.7,117.4$, 
117.3 (q, $J=289.6 \mathrm{~Hz}), 114.7,112.2 ;{ }^{19} \mathrm{~F}$ NMR: -69.47; IR $\left(\mathrm{CDCl}_{3}, \mathrm{~cm}^{-1}\right)$ 3306, 2956, 2918, 2856, 1648, 1572, 1145; HRMS m/z 452.0966 (calcd $\mathrm{C}_{22} \mathrm{H}_{14} \mathrm{~F}_{6} \mathrm{~N}_{2} \mathrm{O}_{2}$, 452.0959).

General procedure for the intermolecular $\mathrm{S}-\mathrm{N}$ addition of sulfinamides to arynes.

To a solution of the $N$-aryltrifluoromethanesulfinamide $(0.5 \mathrm{mmol})$ and silylaryl triflate ( $0.75 \mathrm{mmol}$ ) in THF (6 mL) was slowly added tetra- $n$-butylammonium fluoride (TBAF, $0.9 \mathrm{mmol}$ ). The solution was allowed to stir at room temperature for $30 \mathrm{~min}$ and was then washed with brine $(20 \mathrm{~mL})$ and extracted with diethyl ether $(20 \mathrm{~mL})$. The combined ether fractions were dried over $\mathrm{Na}_{2} \mathrm{SO}_{4}$ and concentrated under reduced pressure. The residue was purified by flash chromatography on silica gel to afford the desired product.<smiles>O=S(c1ccccc1Nc1ccccc1)C(F)(F)F</smiles>

(2-Phenylamino)phenyl trifluoromethyl sulfoxide. The indicated compound was obtained in an $80 \%$ yield (114 mg) as a light yellow oil: ${ }^{1} \mathrm{H}$ NMR (300 MHz, $\left.\mathrm{CDCl}_{3}\right) \delta$ 6.88 (td, $J=7.8,0.9 \mathrm{~Hz}, 1 \mathrm{H}$ ), 7.09 (t, $J=7.2 \mathrm{~Hz}, 1 \mathrm{H}$ ), 7.17 (d, $J=7.5 \mathrm{~Hz}, 2 \mathrm{H}$ ), 7.24-7.42 (m, 5H), 8.17 (s, $1 \mathrm{H}) ;{ }^{13} \mathrm{C}$ NMR (75 MHz, $\left.\mathrm{CDCl}_{3}\right) \delta 148.4,140.5,135.1,129.8,129.7$ (q, $J=1.0 \mathrm{~Hz}), 125.9$ (q, $J=336.1 \mathrm{~Hz}) 124.1,121.9,118.9,116.5,115.7(\mathrm{q}, J=1.6 \mathrm{~Hz}) ;{ }^{19} \mathrm{~F}$ NMR: -71.9; IR ( $\left.\mathrm{CDCl}_{3}, \mathrm{~cm}^{-1}\right)$ 3325, 3059, 3043, 1590, 1472; HRMS m/z 285.0440 (calcd $\mathrm{C}_{13} \mathrm{H}_{10} \mathrm{~F}_{3} \mathrm{NOS}$, 285.0435).<smiles>O=S(c1ccccc1Nc1ccc(I)cc1)C(F)(F)F</smiles>

[2-(4-Iodophenylamino)]phenyl trifluoromethyl sulfoxide. The indicated compound was obtained in an 81\% yield (166 mg) as a light green oil: ${ }^{1} \mathrm{H}$ NMR (400 MHz, $\left.\mathrm{CDCl}_{3}\right) \delta 6.92-9.95$ (m, 3H), 7.29 (d, $J=8.4 \mathrm{~Hz}, 1 \mathrm{H}$ ), 7.39-7.45 (m, 2H), 7.61 (d, $J$ $=8.8 \mathrm{~Hz}, 2 \mathrm{H}), 8.19(\mathrm{~s}, 1 \mathrm{H}) ;{ }^{13} \mathrm{C} \mathrm{NMR}\left(100 \mathrm{MHz}, \mathrm{CDCl}_{3}\right) \delta 147.6,140.4,138.6,135.1$, 129.8 (q, $J=1.0 \mathrm{~Hz}), 125.7$ (q, $J=335.6 \mathrm{~Hz}), 123.1,119.8,116.9,116.4$ (q, $J=1.8 \mathrm{~Hz})$, 86.4; ${ }^{19}$ F NMR: -71.7; IR $\left(\mathrm{CDCl}_{3}, \mathrm{~cm}^{-1}\right)$ 3318, 3060, 3028, 2921, 2850, 1583, 1515; HRMS m/z 410.9410 (calcd $\mathrm{C}_{13} \mathrm{H}_{9} \mathrm{~F}_{3} \mathrm{INOS}$, 410.9402). 
<smiles>COc1ccc(Nc2ccccc2S(=O)C(F)(F)F)cc1</smiles>

[2-(4-Methoxyphenylamino)]phenyl trifluoromethyl sulfoxide. The indicated compound was obtained in a 91\% yield (143 mg) as a light yellow oil: ${ }^{1} \mathrm{H}$ NMR (300 $\left.\mathrm{MHz} \mathrm{CDCl}_{3}\right) \delta 3.81(\mathrm{~s}, 3 \mathrm{H}), 6.78(\mathrm{td}, J=7.8,0.9 \mathrm{~Hz}, 1 \mathrm{H}), 6.90$ (dt, $\left.J=9.0,3.6 \mathrm{~Hz}, 2 \mathrm{H}\right)$, 7.00 (d, $J=8.7 \mathrm{~Hz}, 1 \mathrm{H}$ ), 7.12 (dt, $J=8.7,3.3 \mathrm{~Hz}, 2 \mathrm{H}$ ), 7.30-7.35 (m, 2H), 8.07 (s, 1H); ${ }^{13} \mathrm{C} \mathrm{NMR}\left(75 \mathrm{MHz}, \mathrm{CDCl}_{3}\right) \delta 157.3,150.1,135.1,132.8,129.5$, (q, $\left.J=1.1 \mathrm{~Hz}\right), 126.0$ (q, $J=336.3 \mathrm{~Hz}$ ), 125.7, 117.7, 115.2, 115.0, 113.7 (q, $J=1.6 \mathrm{~Hz}$ ), 55.7; ${ }^{19}$ F NMR: -71.8; IR $\left(\mathrm{CDCl}_{3}, \mathrm{~cm}^{-1}\right)$ 3332, 3003, 2959, 2909, 2836, 1595, 1512; HRMS m/z 315.0546 (calcd $\left.\mathrm{C}_{14} \mathrm{H}_{12} \mathrm{~F}_{3} \mathrm{NO}_{2} \mathrm{~S}, 315.0541\right)$.

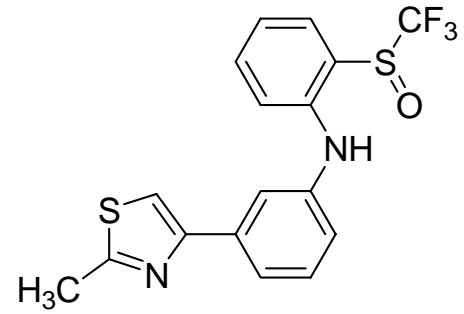

2-[3-(2-Methyl-4-thiazolyl)phenylamino]phenyl trifluoromethyl sulfoxide. The indicated compound was obtained in a 75\% yield (143 mg) as a light yellow solid: $\mathrm{mp}$ 97-98 ${ }^{\circ} \mathrm{C} ;{ }^{1} \mathrm{H}$ NMR (300 MHz, $\mathrm{CDCl}_{3}$ ) $\delta 2.75$ (s, 3H), 6.89 (td, $\left.J=7.5,1.2 \mathrm{~Hz}, 1 \mathrm{H}\right), 7.14$ (dd, $J=7.8,1.2 \mathrm{~Hz}, 1 \mathrm{H}), 7.30$ (s, 1H), 7.32-7.43 (m, 4H), 7.58 (d, $J=7.8 \mathrm{~Hz}, 1 \mathrm{H}), 7.70$ (t, $J=1.8 \mathrm{~Hz}, 1 \mathrm{H}), 8.21(\mathrm{~s}, 1 \mathrm{H}) ;{ }^{13} \mathrm{C}$ NMR $\left(75 \mathrm{MHz}, \mathrm{CDCl}_{3}\right) \delta 166.2,154.7,148.2$, 140.9, 136.3, 135.1, 130.0, 129.7, 125.9 (q, $J=336.0 \mathrm{~Hz}$ ), 122.0, 121.1, 119.9, 119.2, 116.7, 115.9, 113.1, 19.5; ${ }^{19} \mathrm{~F}$ NMR: -71.7; IR $\left(\mathrm{CDCl}_{3}, \mathrm{~cm}^{-1}\right)$ 3323, 3109, 3062, 2924, 1587, 1501; HRMS m/z 382.0426 (calcd $\mathrm{C}_{17} \mathrm{H}_{13} \mathrm{~F}_{3} \mathrm{~N}_{2} \mathrm{OS}_{2}$, 382.0421).<smiles>COc1cc(Nc2ccc(Br)cc2)c(S(=O)C(F)(F)F)cc1OC</smiles>

[2-(4-Bromophenylamino)-4,5-dimethoxy]phenyl trifluoromethyl sulfoxide. The indicated compound was obtained in a 72\% yield (152 mg) as a white solid: $\mathrm{mp} 132-133$ ${ }^{\circ} \mathrm{C} ;{ }^{1} \mathrm{H}$ NMR (300 MHz, $\left.\mathrm{CDCl}_{3}\right) \delta 3.82$ (s, 3H), 3.88 (s, 3H), 6.81 (s, 1H), 6.92 (d, $J=$ 
$9.0 \mathrm{~Hz}, 2 \mathrm{H}), 7.01$ (s, 1H), 7.19 (s, 1H), 7.90 (d, $J=9.0 \mathrm{~Hz}, 2 \mathrm{H}) ;{ }^{13} \mathrm{C}$ NMR $(75 \mathrm{MHz}$, $\left.\mathrm{CDCl}_{3}\right) \delta 154.9,144.8,141.5,141.1,132.7,125.6$ (q, $\left.J=336.0 \mathrm{~Hz}\right), 120.7,114.7,111.4$ (q, $J=1.6 \mathrm{~Hz}), 110.3,103.4,56.8,56.4 ;{ }^{19} \mathrm{~F}$ NMR: -72.8; IR $\left(\mathrm{CDCl}_{3}, \mathrm{~cm}^{-1}\right)$ 3332, 3065, 3007, 2963, 2911, 2835, 1590, 1489; HRMS m/z 423.9756 (calcd $\mathrm{C}_{15} \mathrm{H}_{13} \mathrm{BrF}_{3} \mathrm{NO}_{3} \mathrm{~S}$, 423.9751).<smiles>Cc1cc(Nc2ccc(Cl)cc2)c(S(=O)C(F)(F)F)cc1C</smiles>

[2-(4-Chlorophenylamino)-4,5-dimethyl]phenyl trifluoromethyl sulfoxide. The indicated compound was obtained in a $55 \%$ yield (96 mg) as a white solid: $\mathrm{mp} 69-71{ }^{\circ} \mathrm{C}$; ${ }^{1} \mathrm{H}$ NMR (300 MHz, $\mathrm{CDCl}_{3}$ ) $\delta 2.22$ (s, 3H), 2.23 (s, 3H), 7.03-7.08 (m, 3H), 7.16 (s, 1H), 7.25-7.28 (m, 2H), 7.81 (s, $1 \mathrm{H}) ;{ }^{13} \mathrm{C}$ NMR (100 MHz, $\left.\mathrm{CDCl}_{3}\right) \delta 145.3,145.2,129.8$ (q, $J$ $=1.0 \mathrm{~Hz}$ ), 129.7, 129.0, 127.9, 125.7 (q, $J=335.9 \mathrm{~Hz}), 121.9,118.6,114.2$ (q, $J=1.5$ $\mathrm{Hz}), 20.7,19.1 ;{ }^{19} \mathrm{~F}$ NMR: -72.1; IR $\left(\mathrm{CDCl}_{3}, \mathrm{~cm}^{-1}\right)$ 3324, 2972, 2860, 1593, 1492; HRMS $\mathrm{m} / \mathrm{z} 347.0362$ (calcd $\mathrm{C}_{15} \mathrm{H}_{13} \mathrm{ClF}_{3} \mathrm{NOS}$, 347.0358).<smiles>O=C(Nc1ccc(Nc2ccccc2S(=O)C(F)(F)F)cc1)C(F)(F)F</smiles>

[2-(4-Trifluoroacetamidophenylamino)]phenyl trifluoromethyl sulfoxide. The indicated compound was obtained in a 58\% yield (114 mg) as a light yellow solid: $\mathrm{mp}$ 101-103 ${ }^{\circ} \mathrm{C}$; ${ }^{1} \mathrm{H}$ NMR $\left(300 \mathrm{MHz}, \mathrm{CDCl}_{3}\right) \delta 6.93(\mathrm{t}, J=7.2 \mathrm{~Hz}, 1 \mathrm{H}), 7.18(\mathrm{~d}, J=8.7 \mathrm{~Hz}$, 2H), 7.28 (d, $J=8.1 \mathrm{~Hz}, 1 \mathrm{H}$ ), 7.42 (q, $J=6.9 \mathrm{~Hz}, 2 \mathrm{H}), 7.54$ (d, $J=8.7 \mathrm{~Hz}, 2 \mathrm{H}$ ), 8.06 (s, $1 \mathrm{H}), 8.24(\mathrm{~s}, 1 \mathrm{H}) ;{ }^{13} \mathrm{C}$ NMR (75 MHz, $\left.\mathrm{CDCl}_{3}\right) \delta 154.9$ (q, $\left.J=47.1 \mathrm{~Hz}\right), 147.9,138.7$, 135.2, 130.9, 129.8 (q, $J=1.0 \mathrm{~Hz}$ ), 125.7 (q, $J=335.8 \mathrm{~Hz}), 122.3,122.2,119.5,117.9$, 116.0 (q, $J=286.9 \mathrm{~Hz}), 115.9$ (q, $J=1.6 \mathrm{~Hz}) ;{ }^{19} \mathrm{~F} \mathrm{NMR:} \mathrm{-71.7,} \mathrm{-76.0;} \mathrm{IR}\left(\mathrm{CDCl}_{3}, \mathrm{~cm}^{-1}\right)$; HRMS m/z 396.0373 (calcd $\mathrm{C}_{15} \mathrm{H}_{10} \mathrm{~F}_{6} \mathrm{~N}_{2} \mathrm{O}_{2} \mathrm{~S}, 396.0367$ ). 


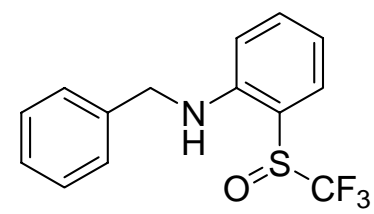

(2-Benzylamino)phenyl trifluoromethyl sulfoxide. The indicated compound was obtained in a $41 \%$ yield as a slightly yellow oil; ${ }^{1} \mathrm{H}$ NMR (300 MHz, $\left.\mathrm{CDCl}_{3}\right) \delta 4.41$ (d, $J$ = 5.4 Hz, 2H), 6.63-6.73 (m, 2H), 7.06 (s, 1H), 7.24-7.36 (m, 7H); ${ }^{13} \mathrm{C}$ NMR (75 MHz, $\left.\mathrm{CDCl}_{3}\right) \delta 151.2,138.3,135.4,129.6(\mathrm{q}, J=1.0 \mathrm{~Hz}), 129.0,127.6,127.1,126.2(\mathrm{q}, J=$ $336.3 \mathrm{~Hz}), 116.1,112.4,112.4$ (q, $J=1.7 \mathrm{~Hz}), 47.3 ;{ }^{19} \mathrm{~F}$ NMR: -71.8; IR $\left(\mathrm{CDCl}_{3}, \mathrm{~cm}^{-1}\right)$ 3375, 3086, 3067, 3034, 2856, 1605, 1527; HRMS m/z 299.0596 (calcd $\mathrm{C}_{14} \mathrm{H}_{12} \mathrm{~F}_{3} \mathrm{NOS}$, 299.0591).

\section{References}

(1) (a) Pena, D.; Perez, D.; Guitian, E.; Castedo, L. J. Am. Chem. Soc. 1999, 121, 5827. (b) Yoshida, H.; Sugiura, S.; Kunai, A. Org. Lett. 2002, 4, 2767. (c) ) Yoshida, H.; Ikadai, J.; Shudo, M.; Ohshita, J.; Kunai, A. . Am. Chem. Soc. 2003, $125,6638$.

(2) Liu, Z.; Larock, R. C. Org. Lett. 2003, 5, 4673.

(3) Billard, T.; Greiner, A.; Langlois, B. R. Tetrahedron 1999, 55, 7243. 


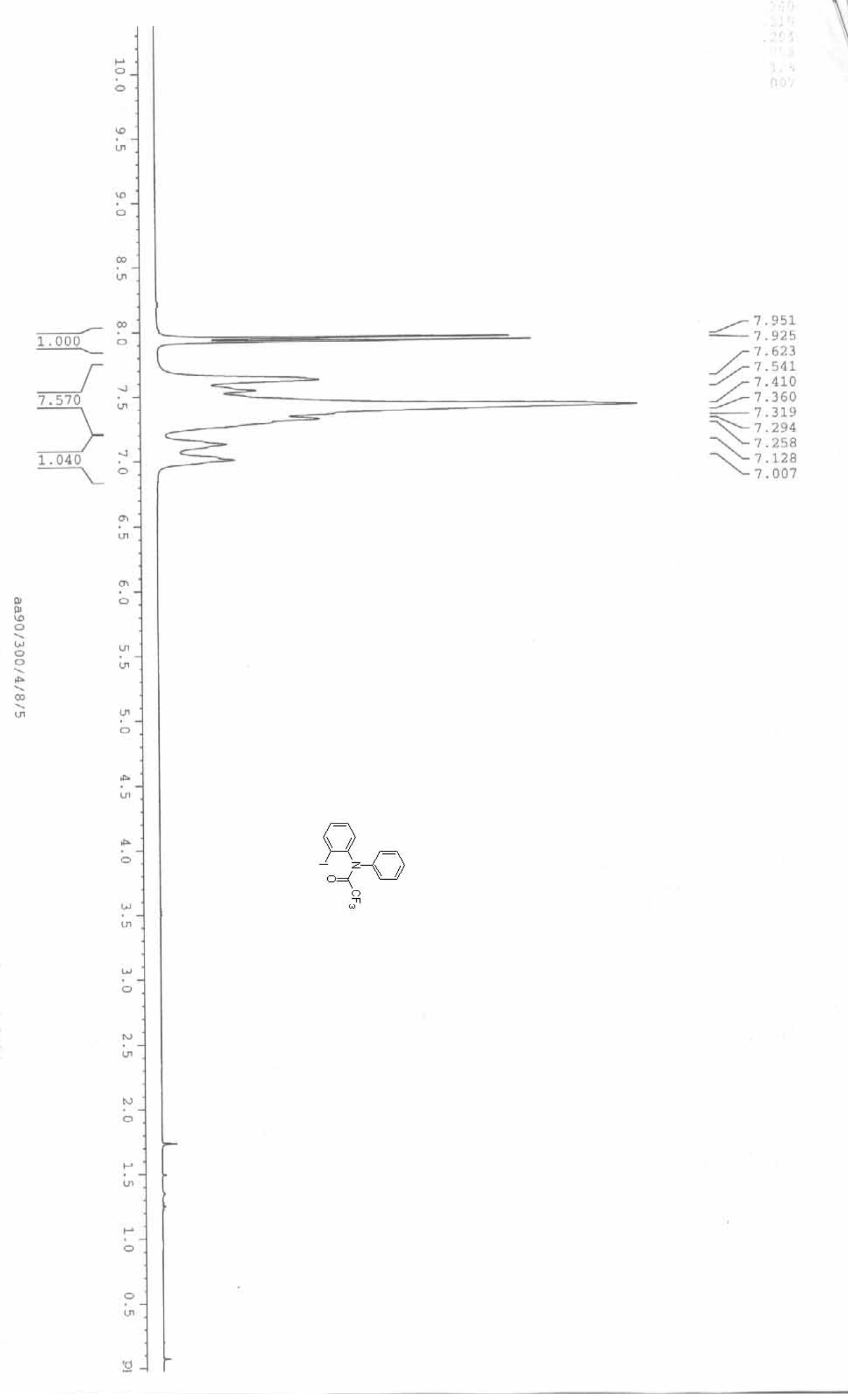




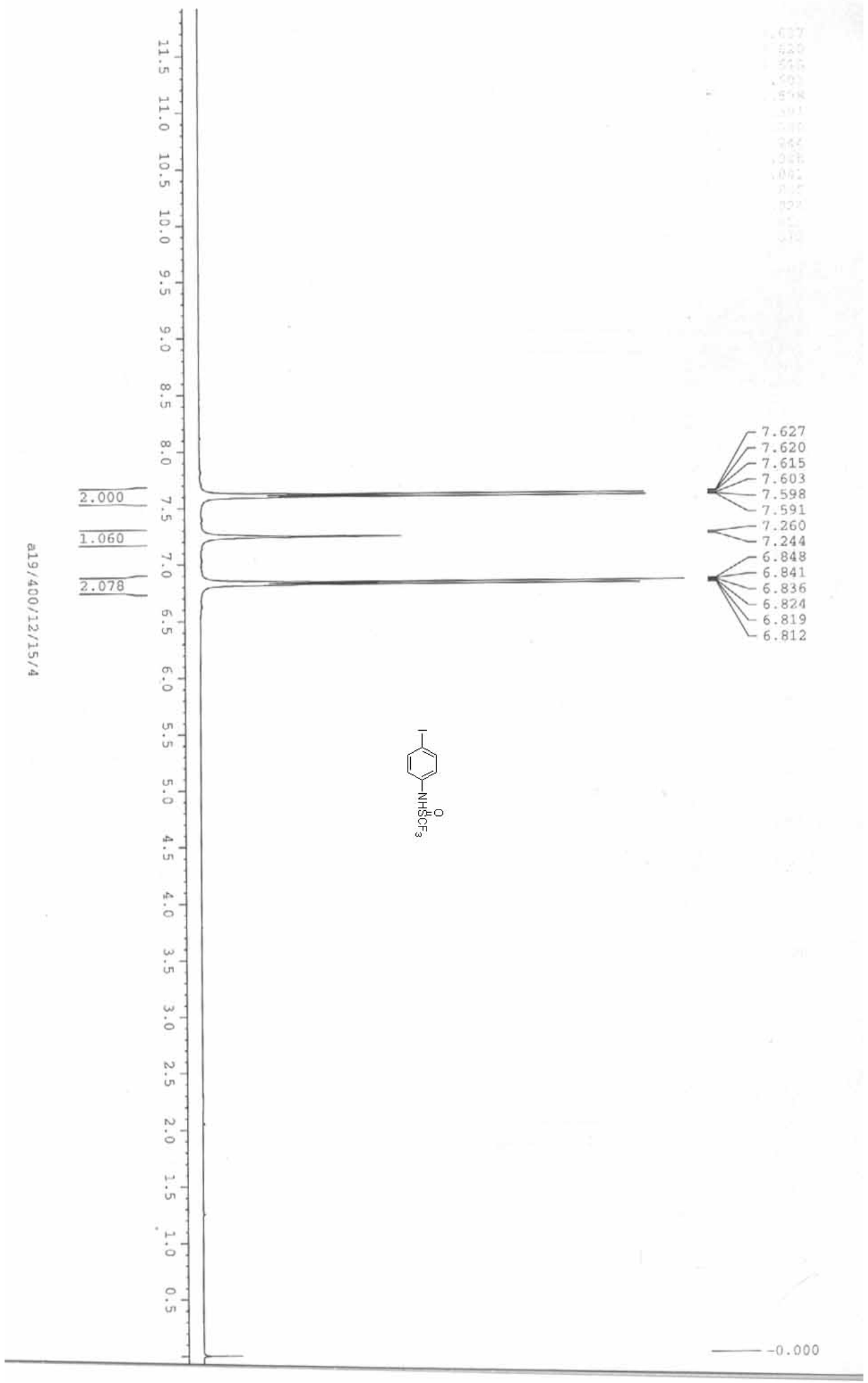




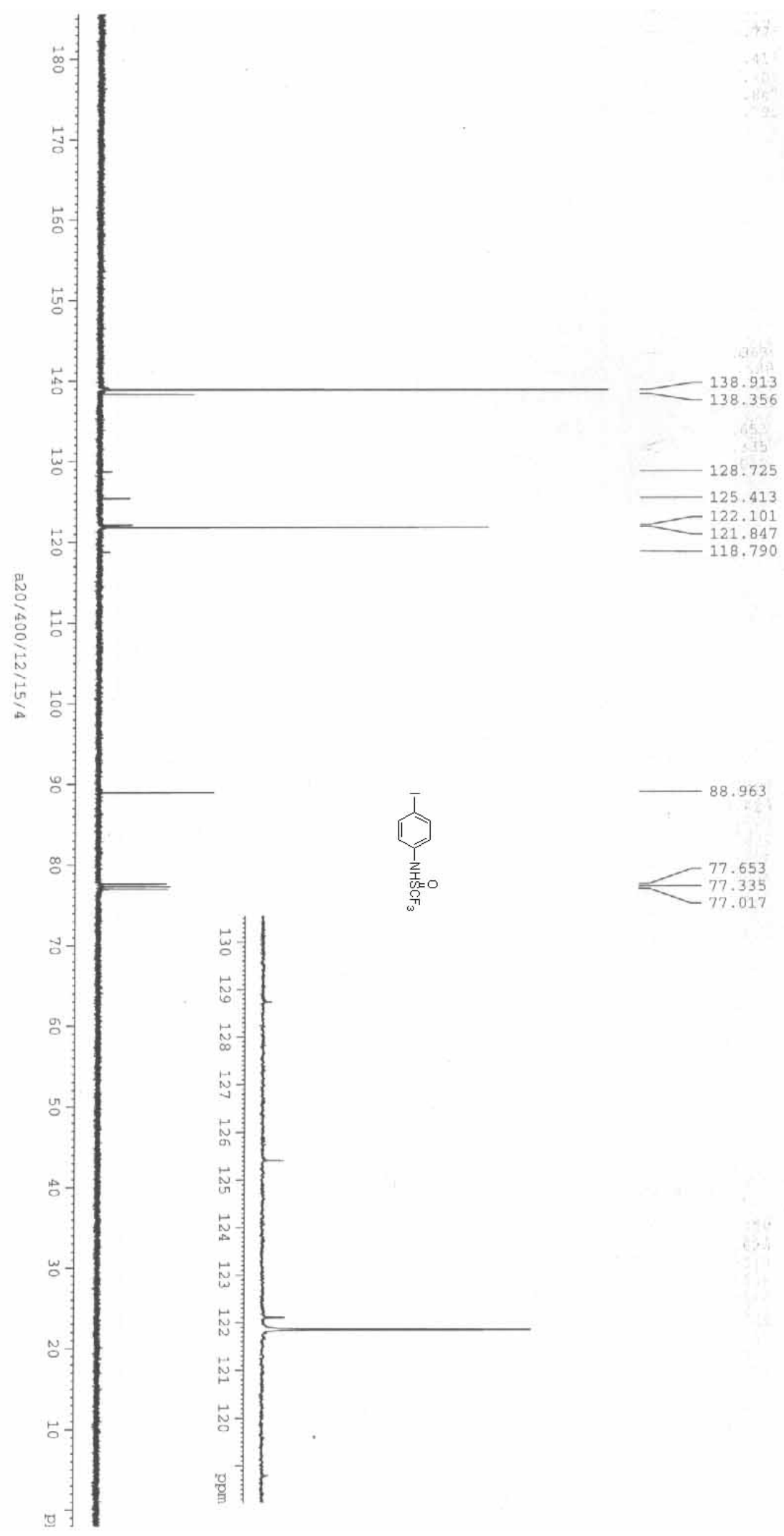




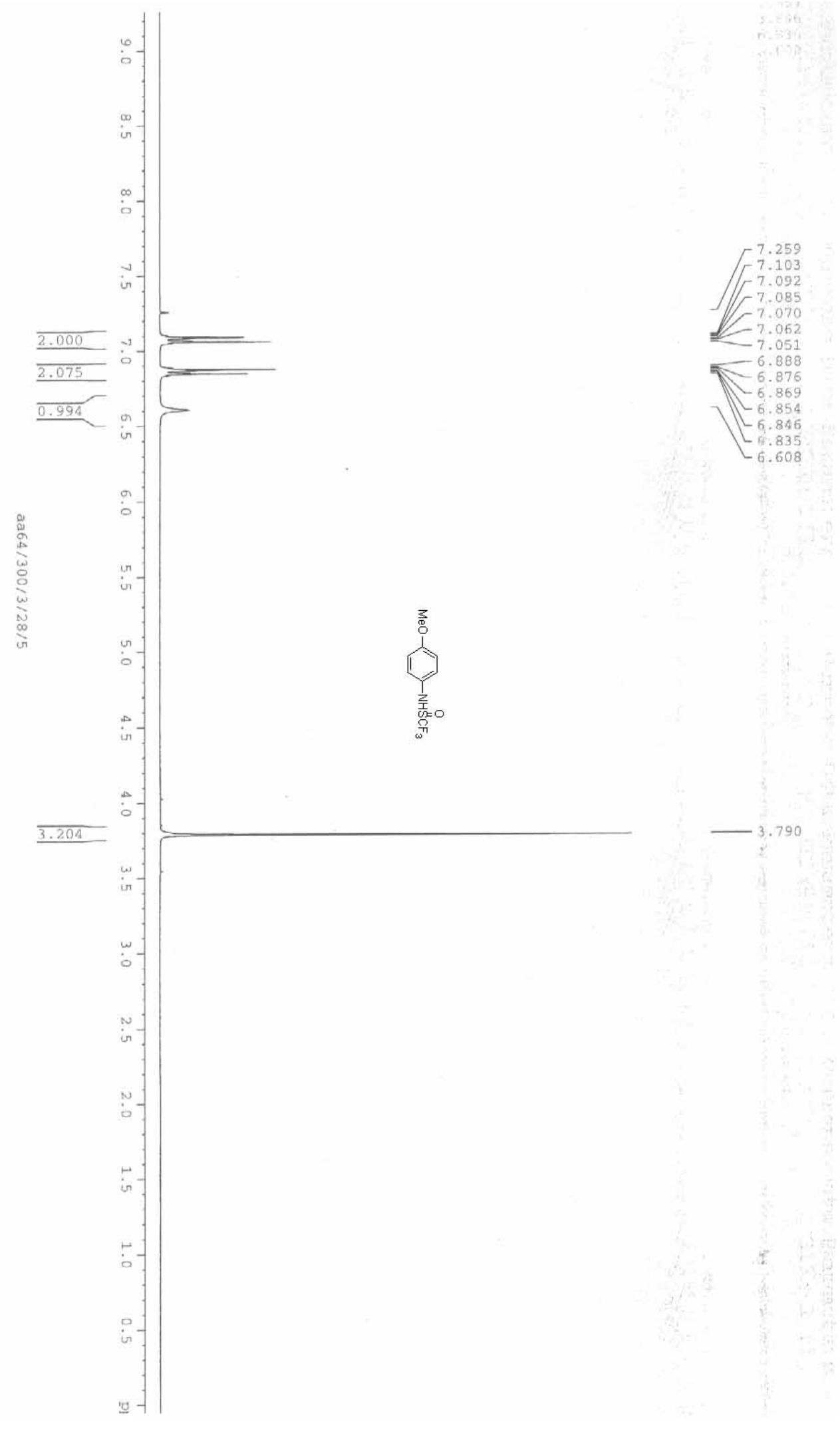




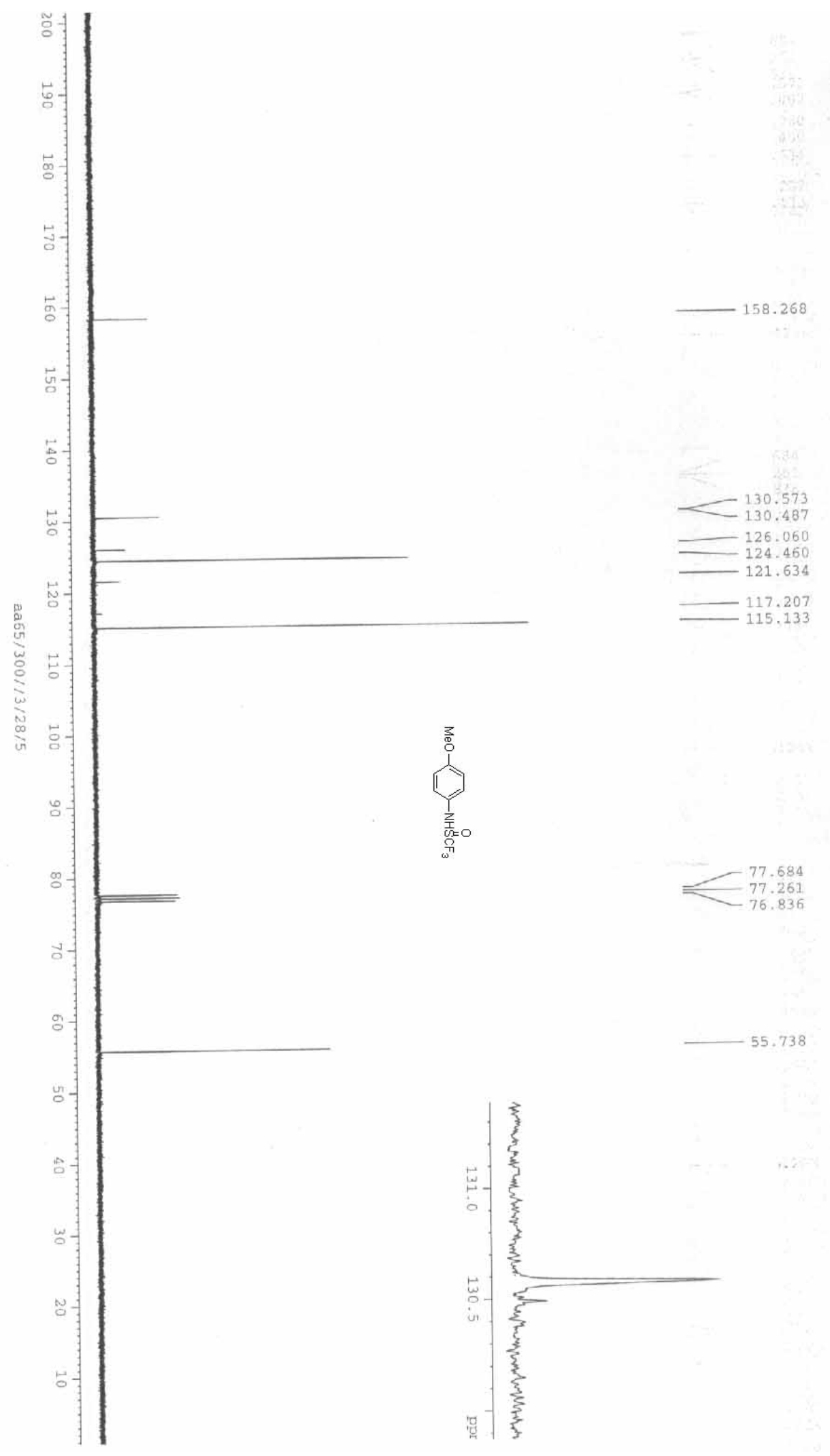




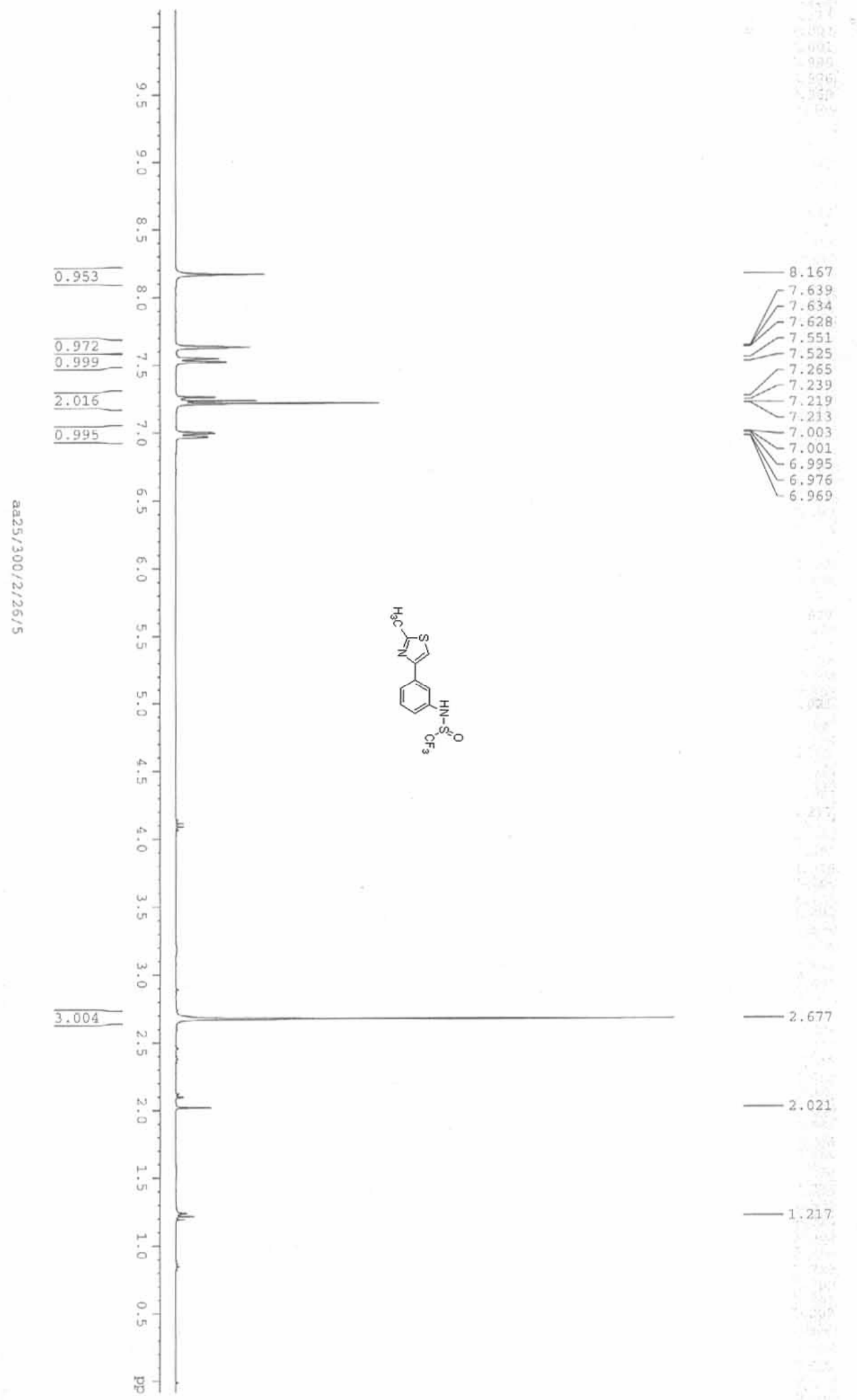




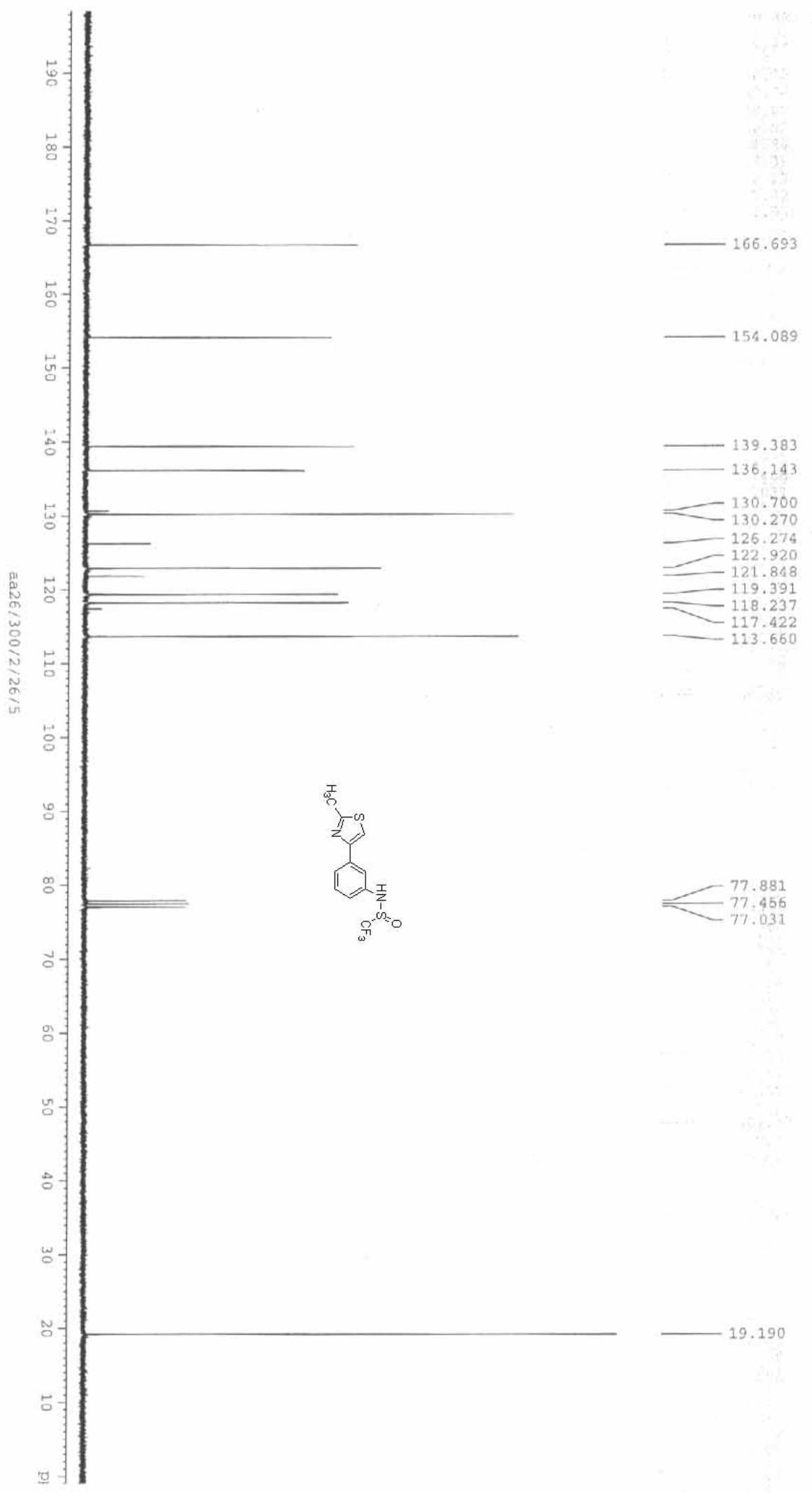



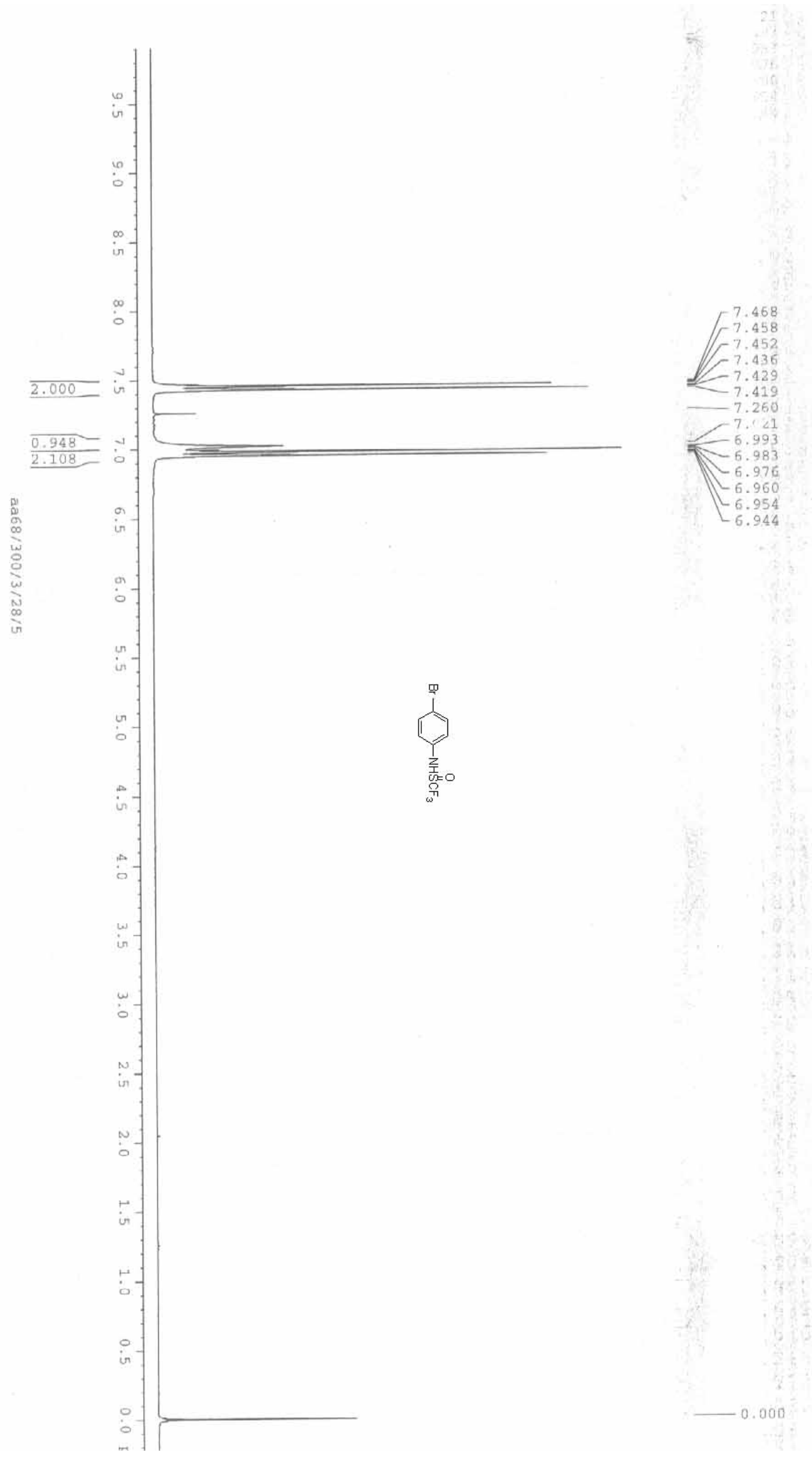


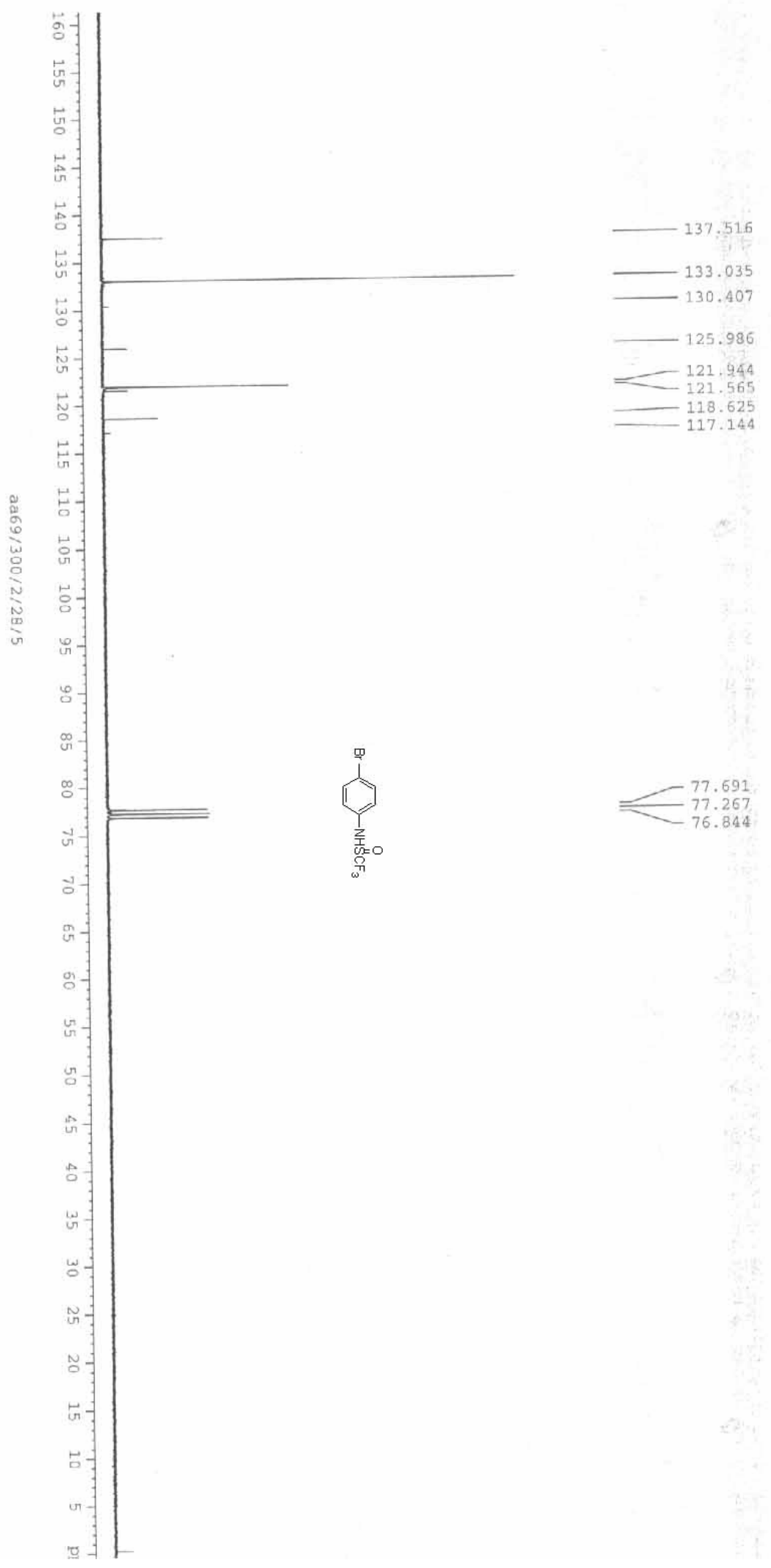



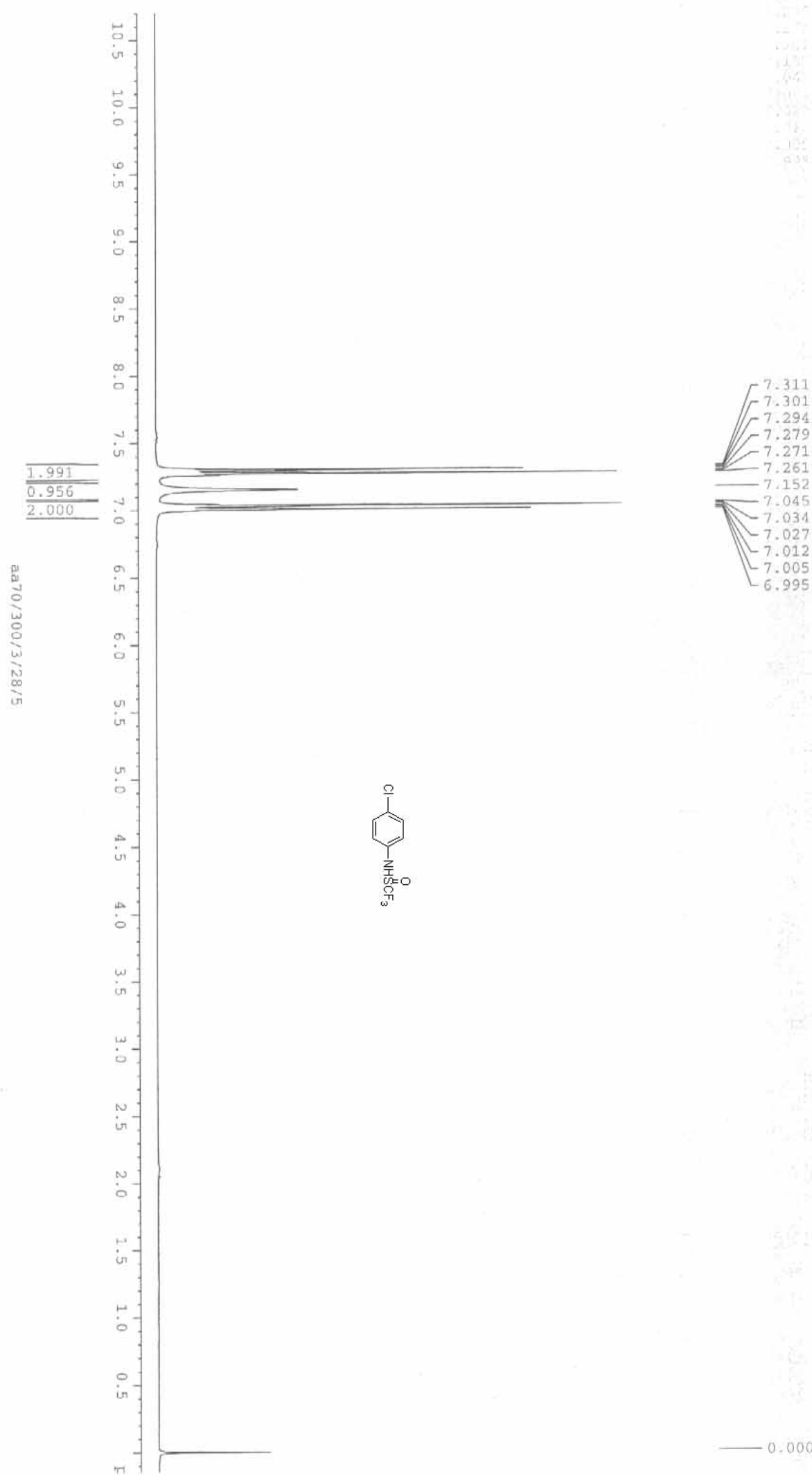

0.000 

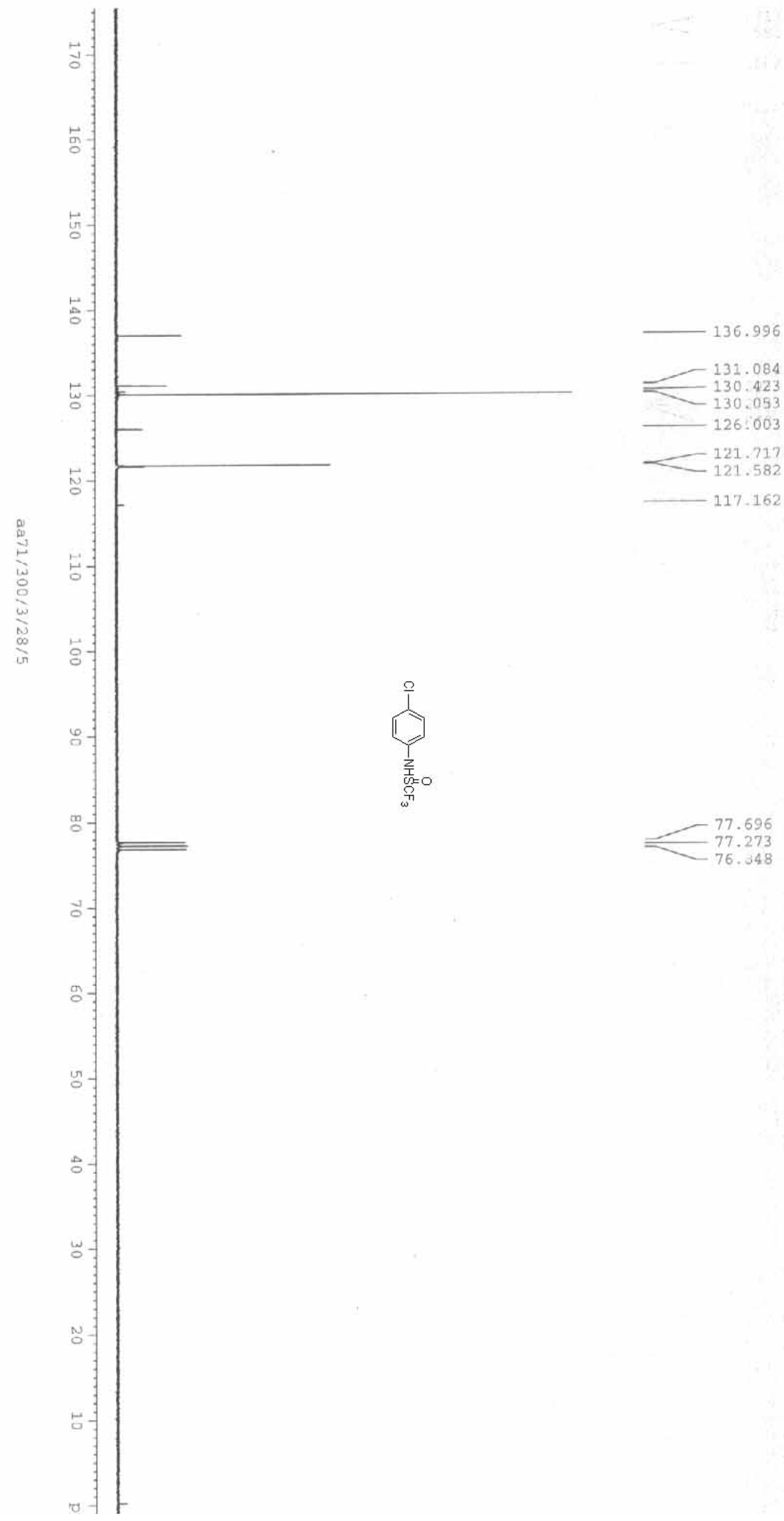


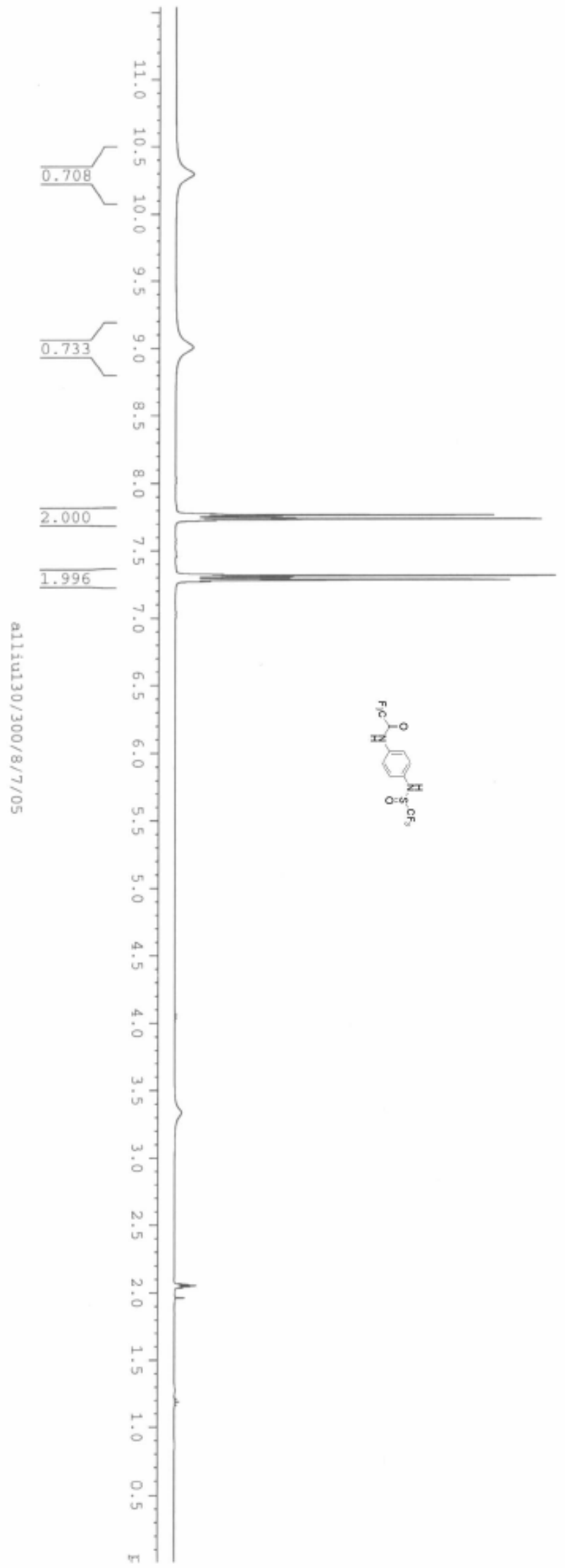

$-10.298$
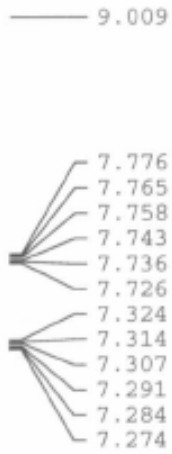

$-2.058$

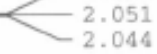



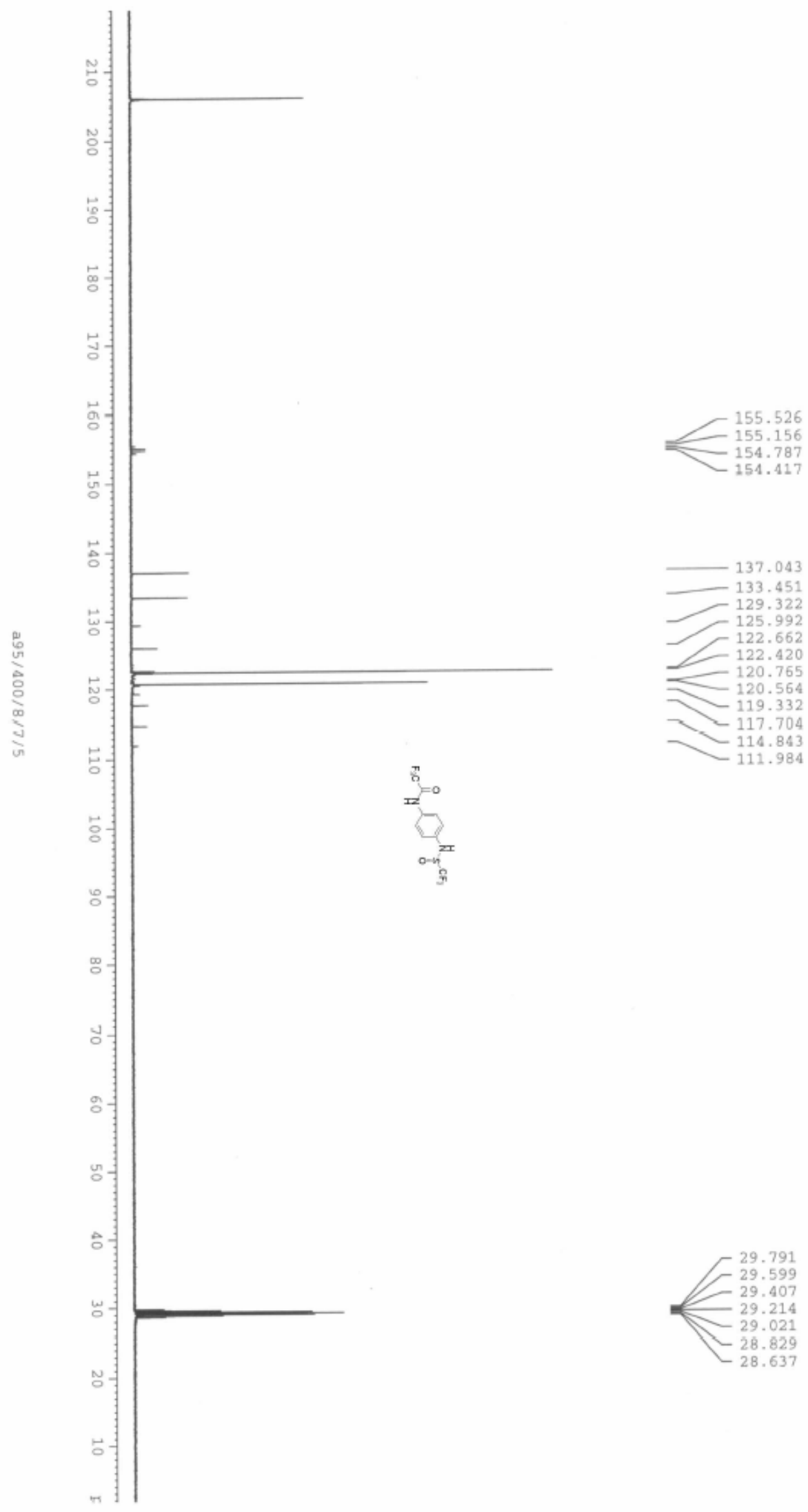


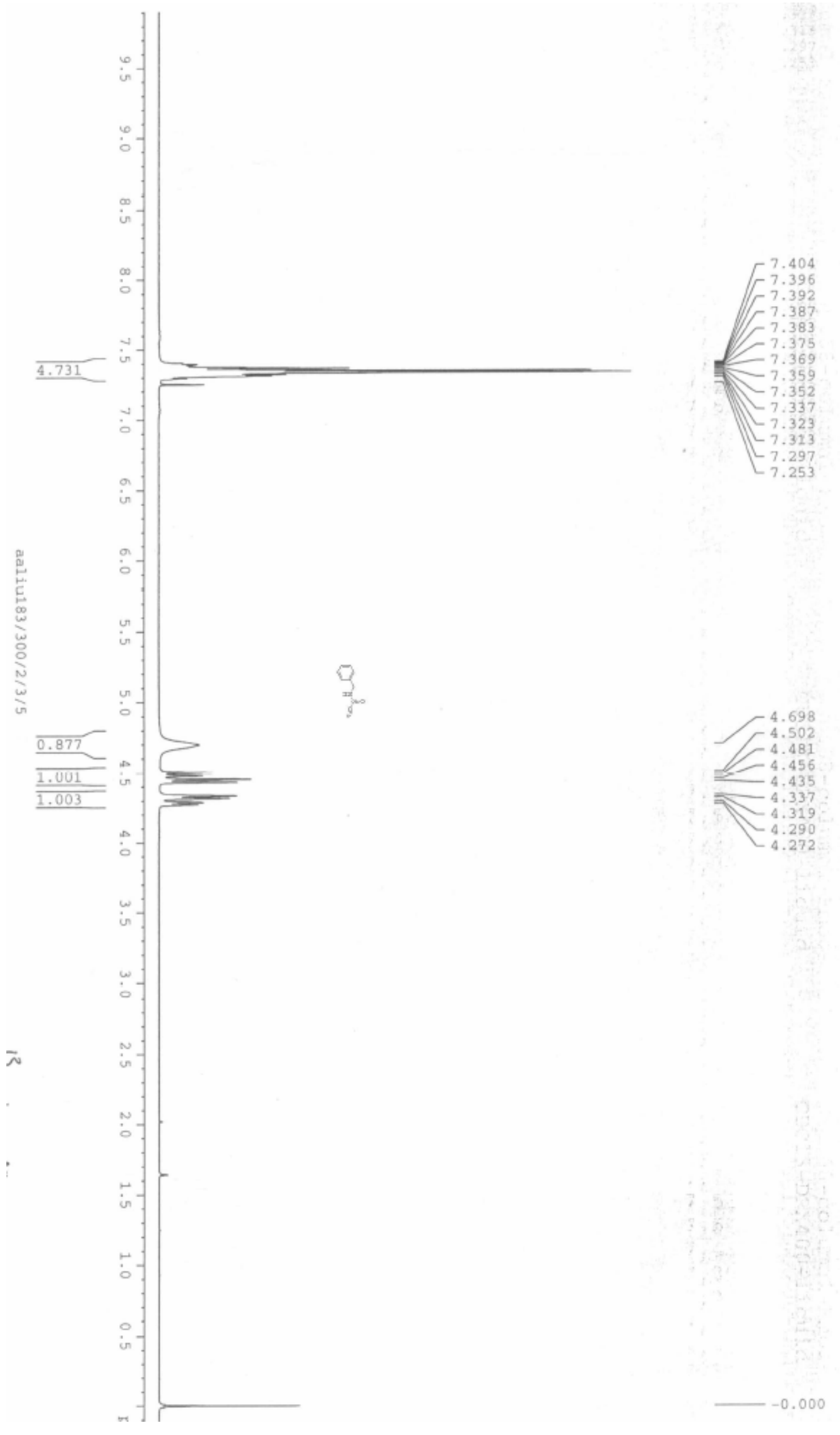




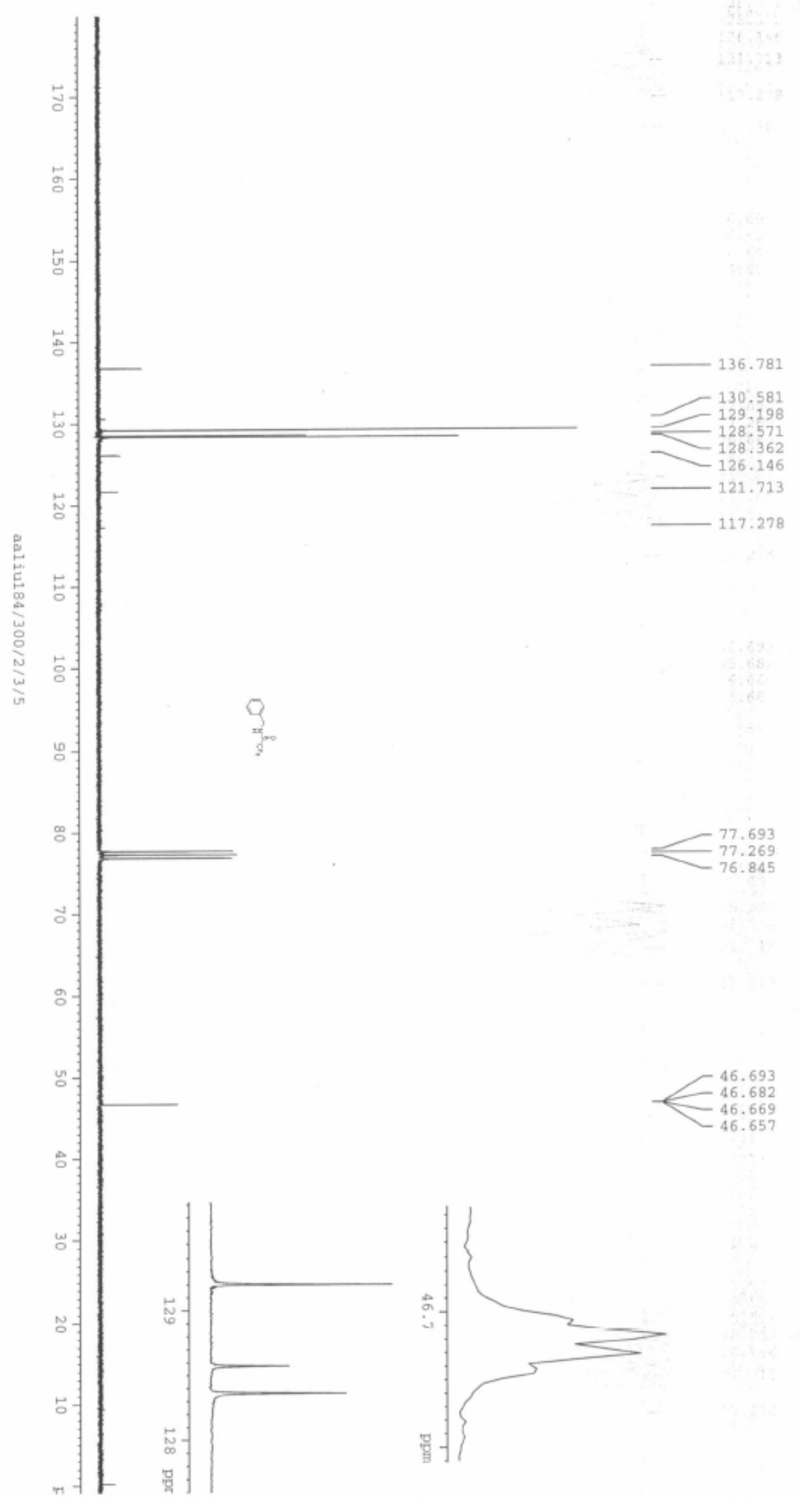




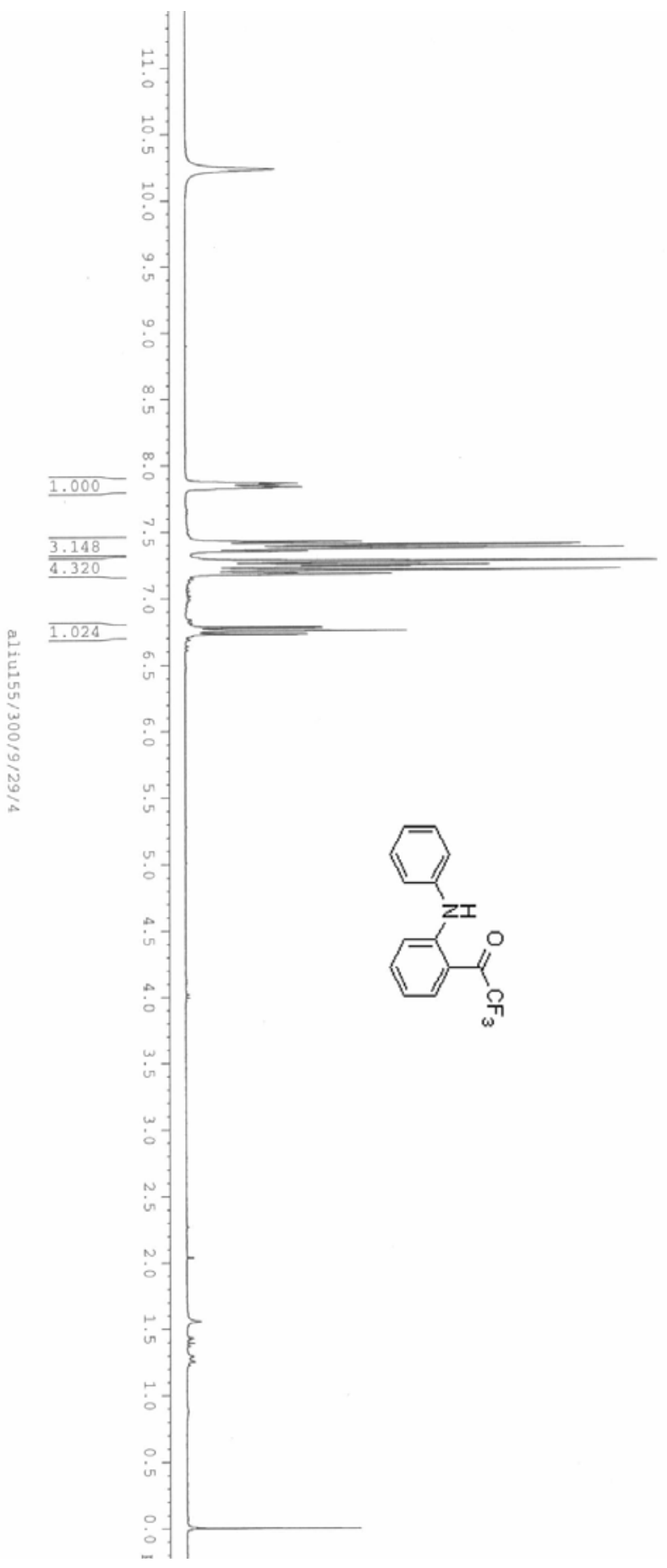

$-10.237$

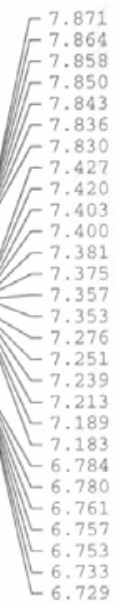




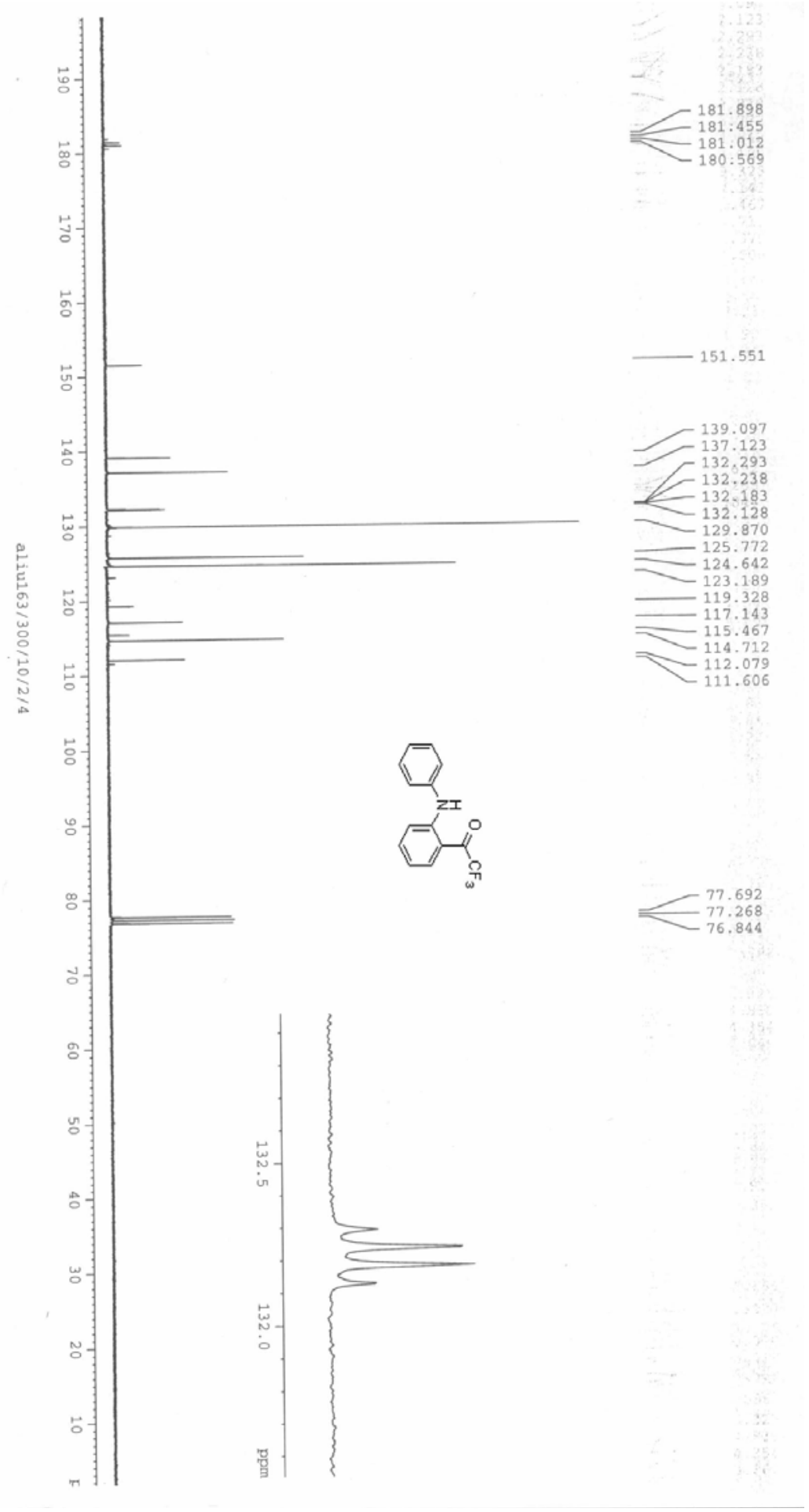




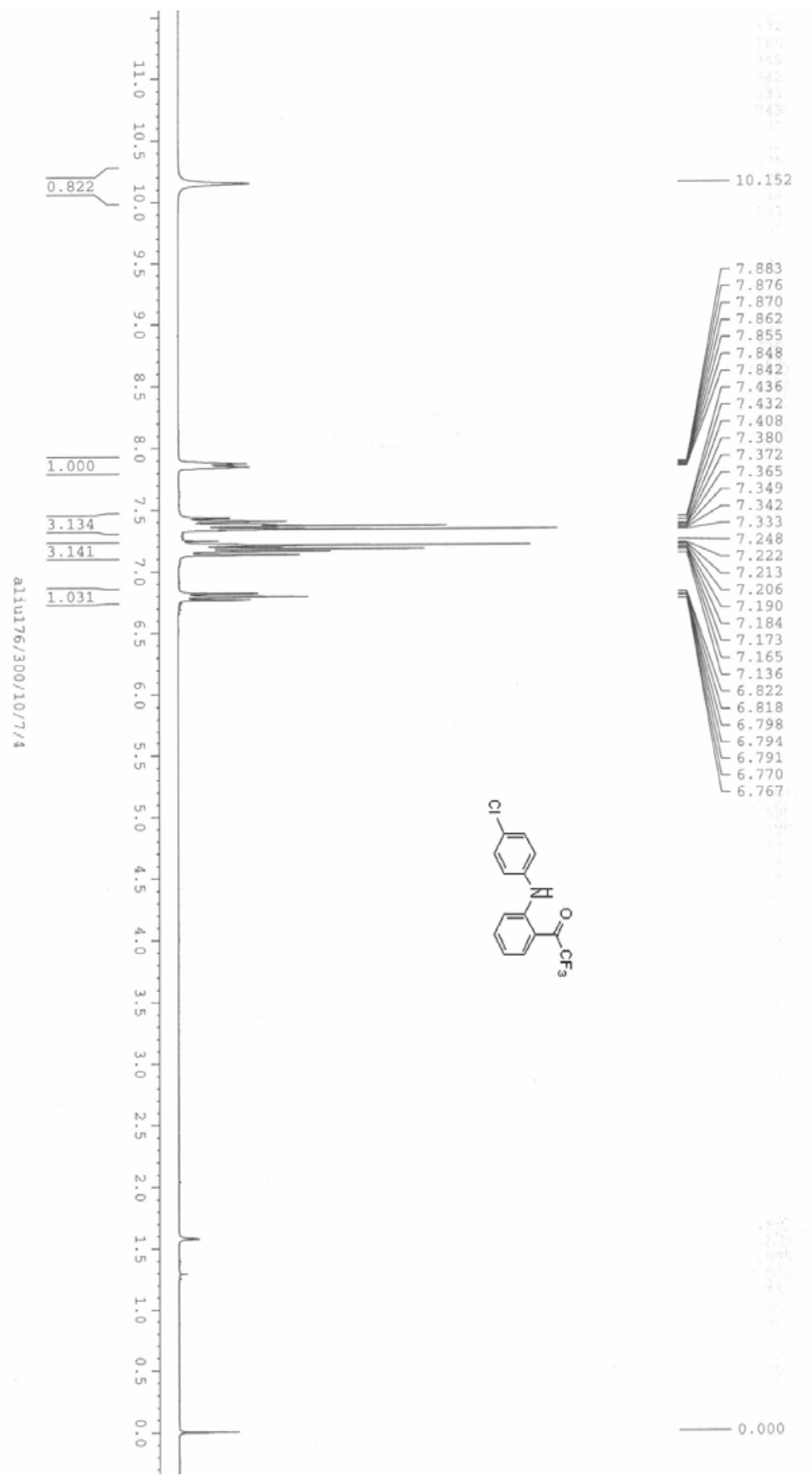




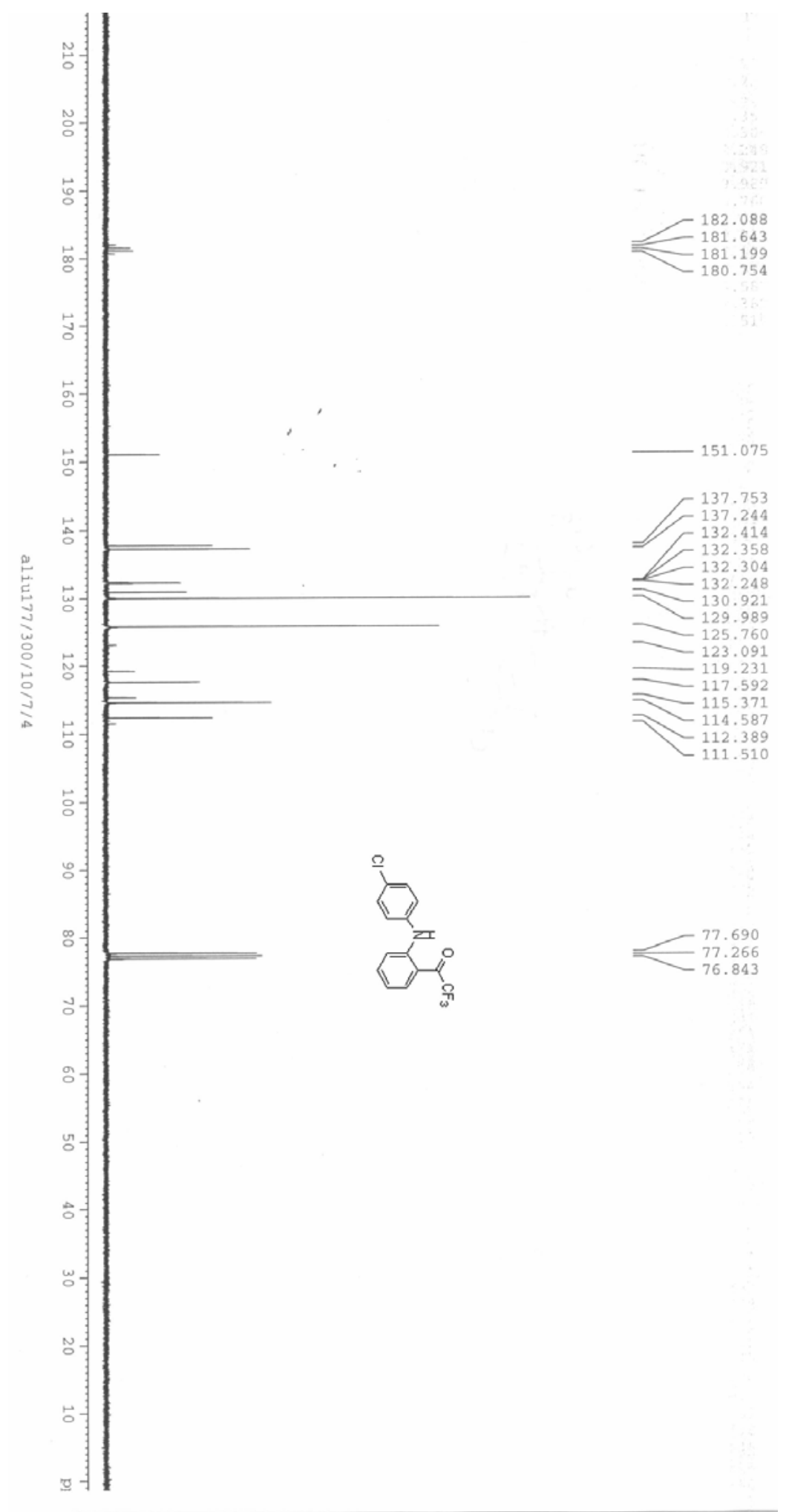




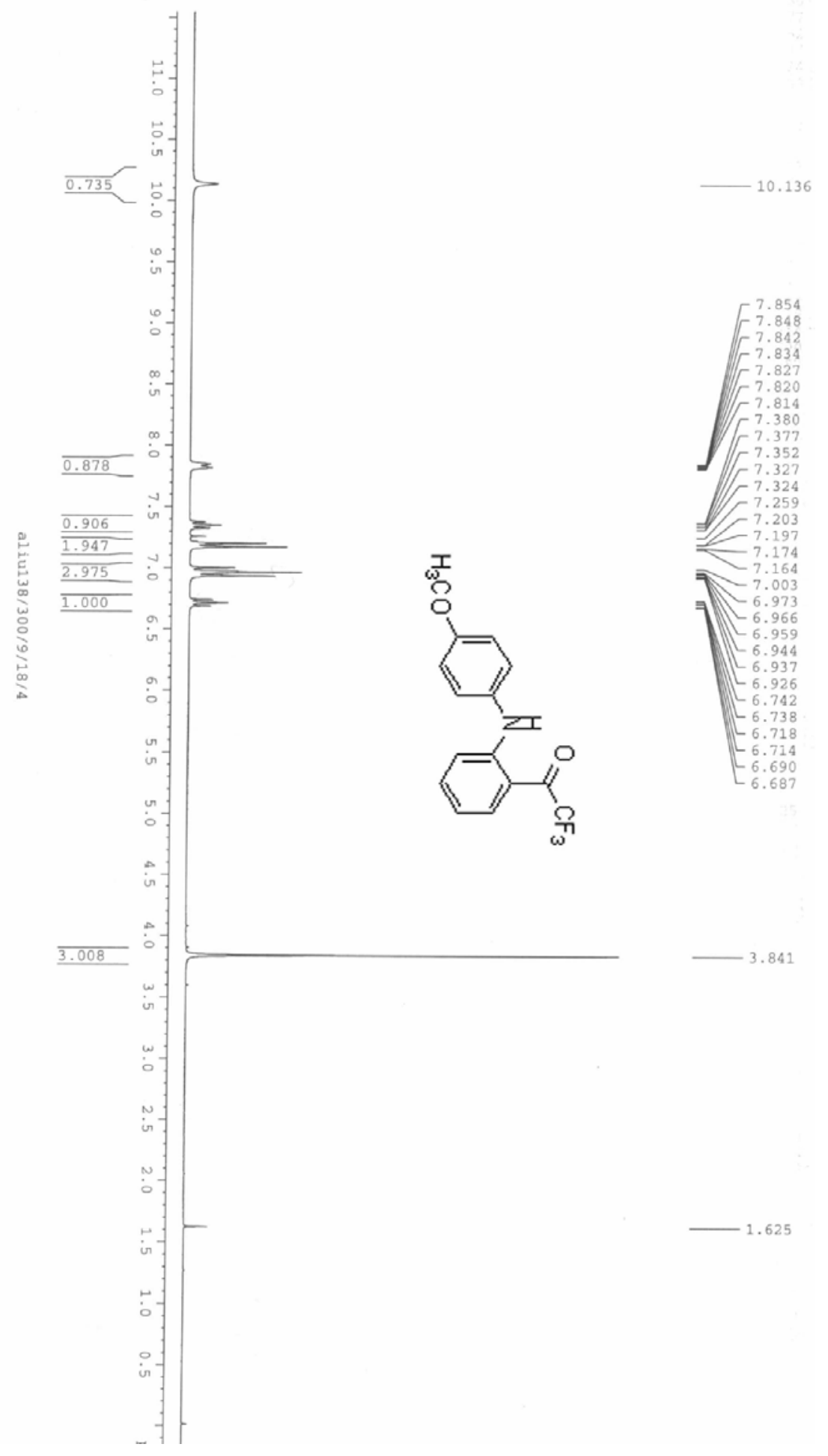



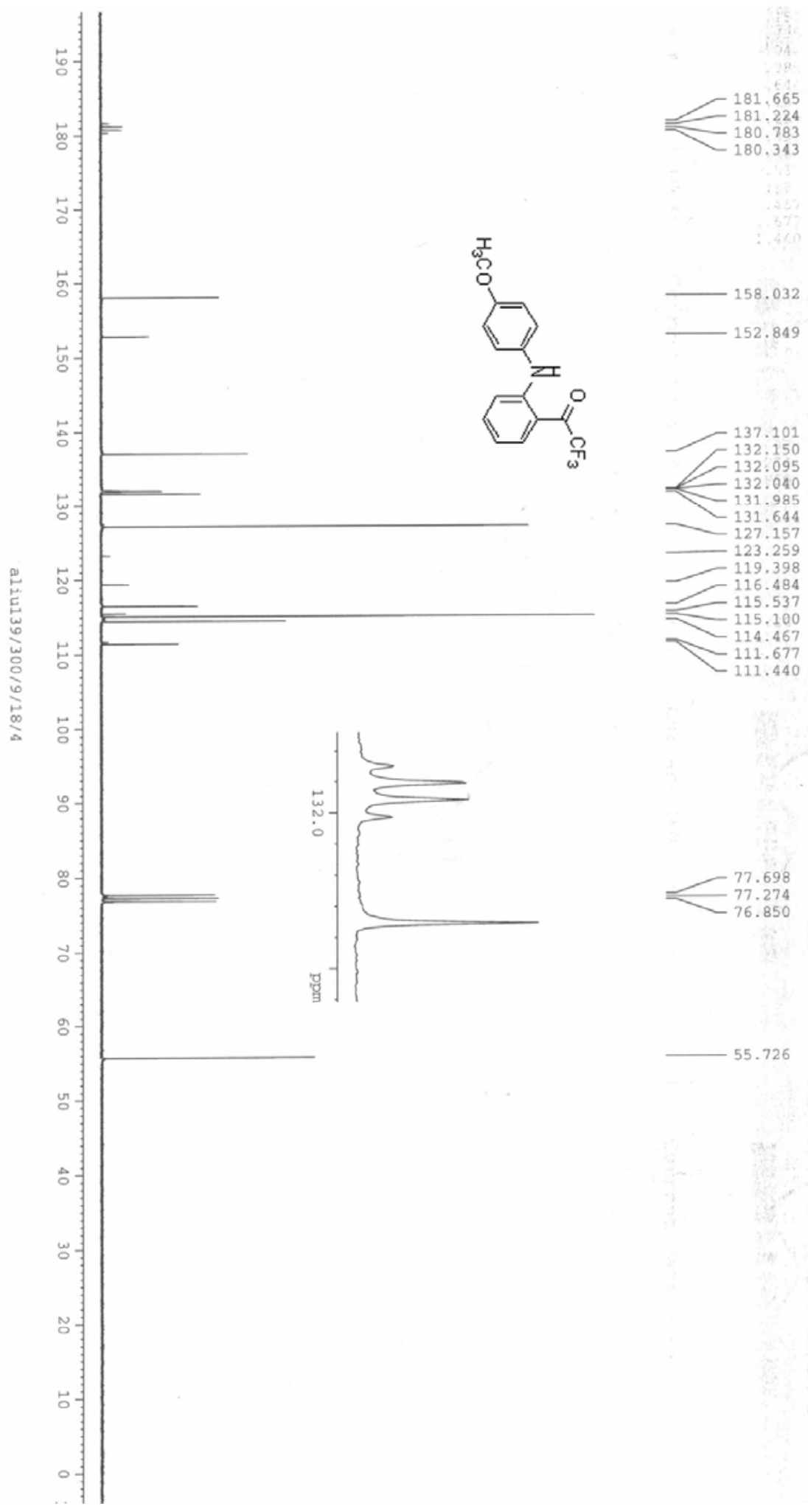


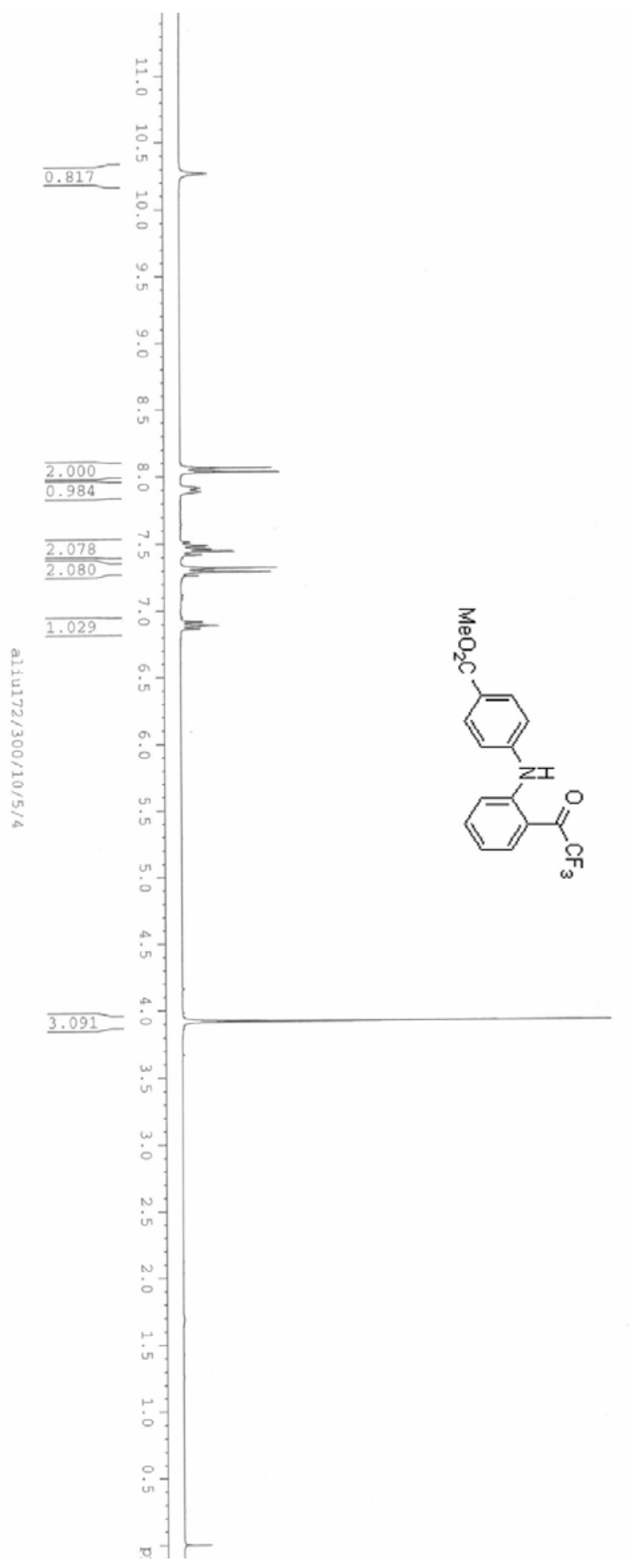

$-10.265$
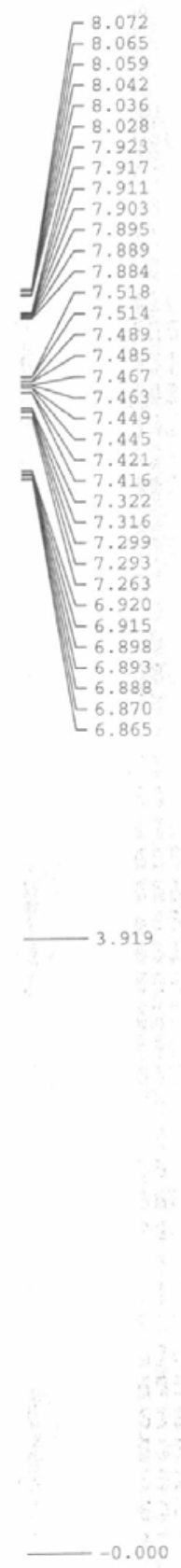

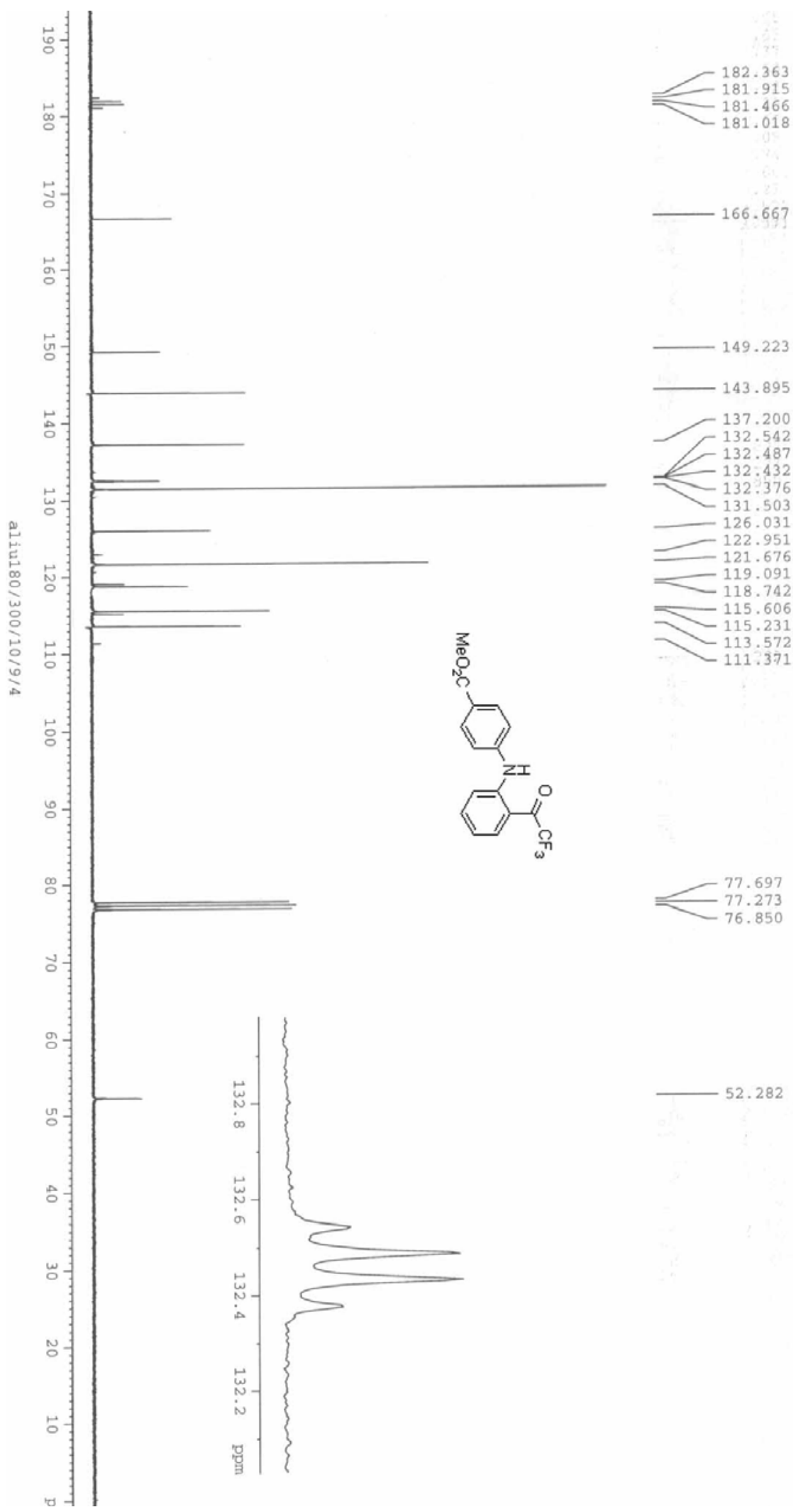

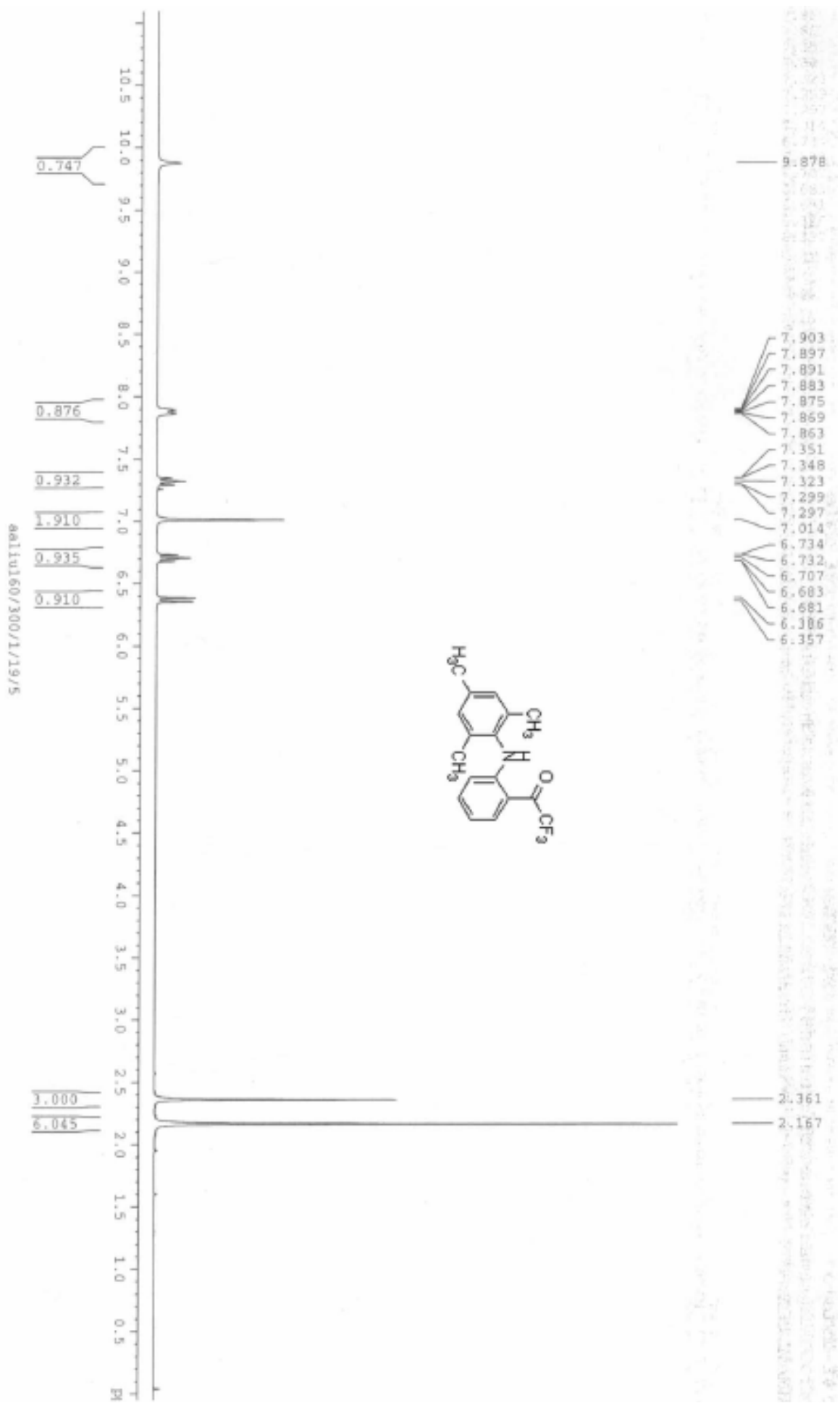

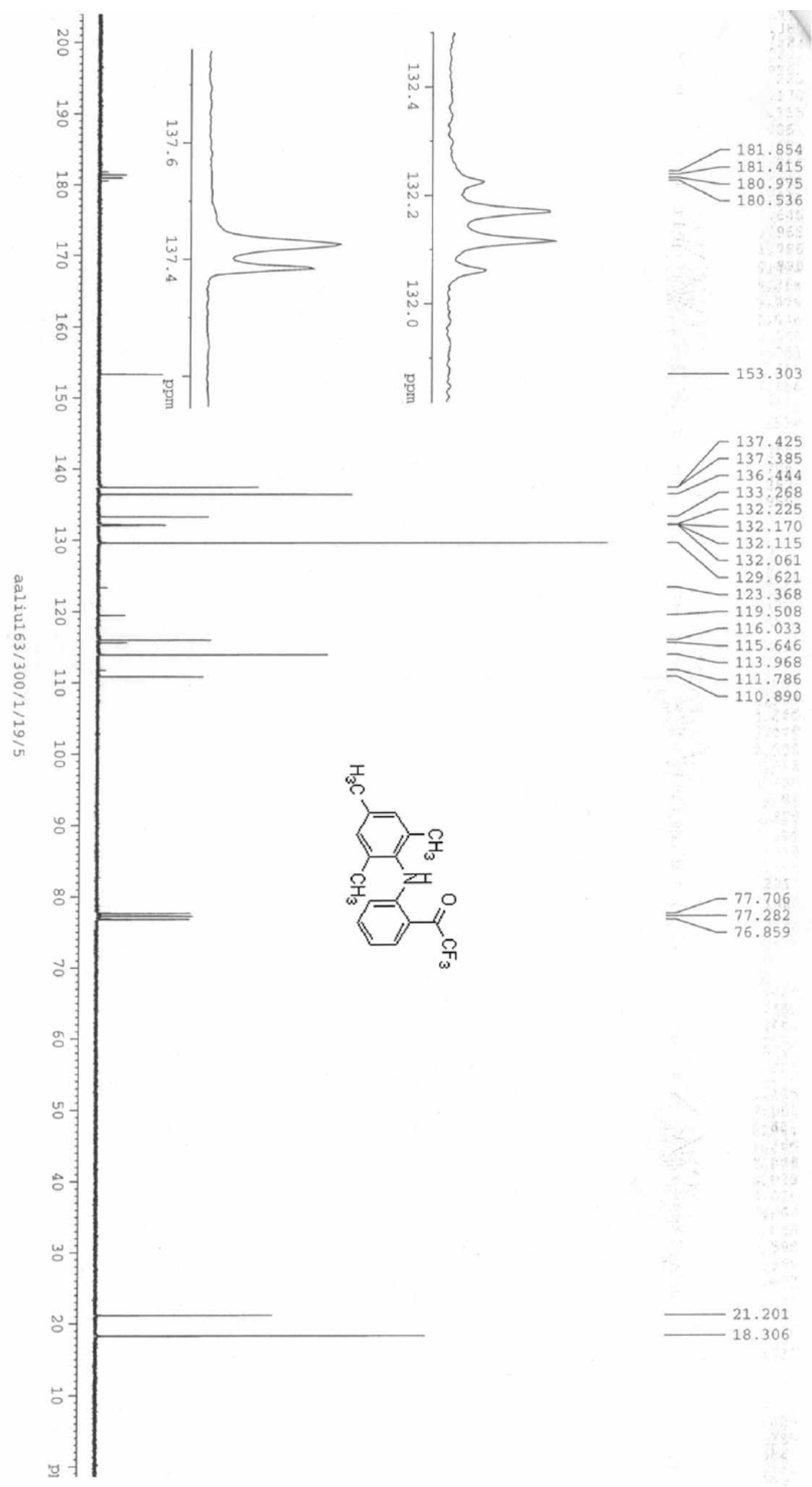

$-21.201$ 


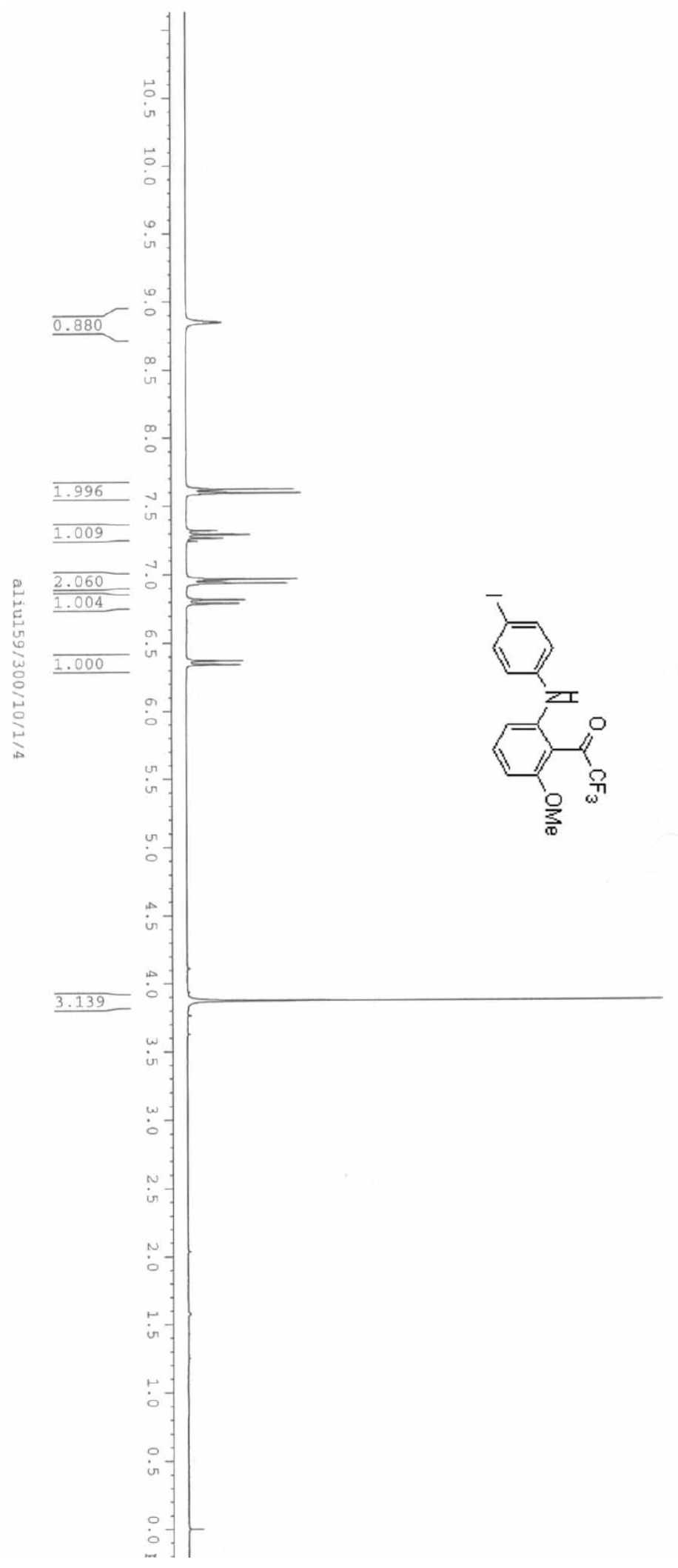

$-8.849$

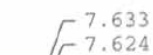

F 7.617

- 7.601

- -7.386

$-7.266$

$-7.244$

-6.975
-6.960

$7-6.960$

$\left[\begin{array}{r}-6.937 \\ -6.937 \\ 6.928\end{array}\right.$

ᄂ 6.819

$-6.791$

$-6.371$

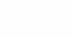

3.873
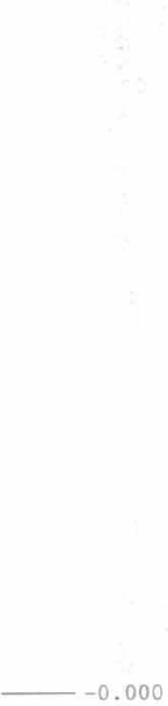


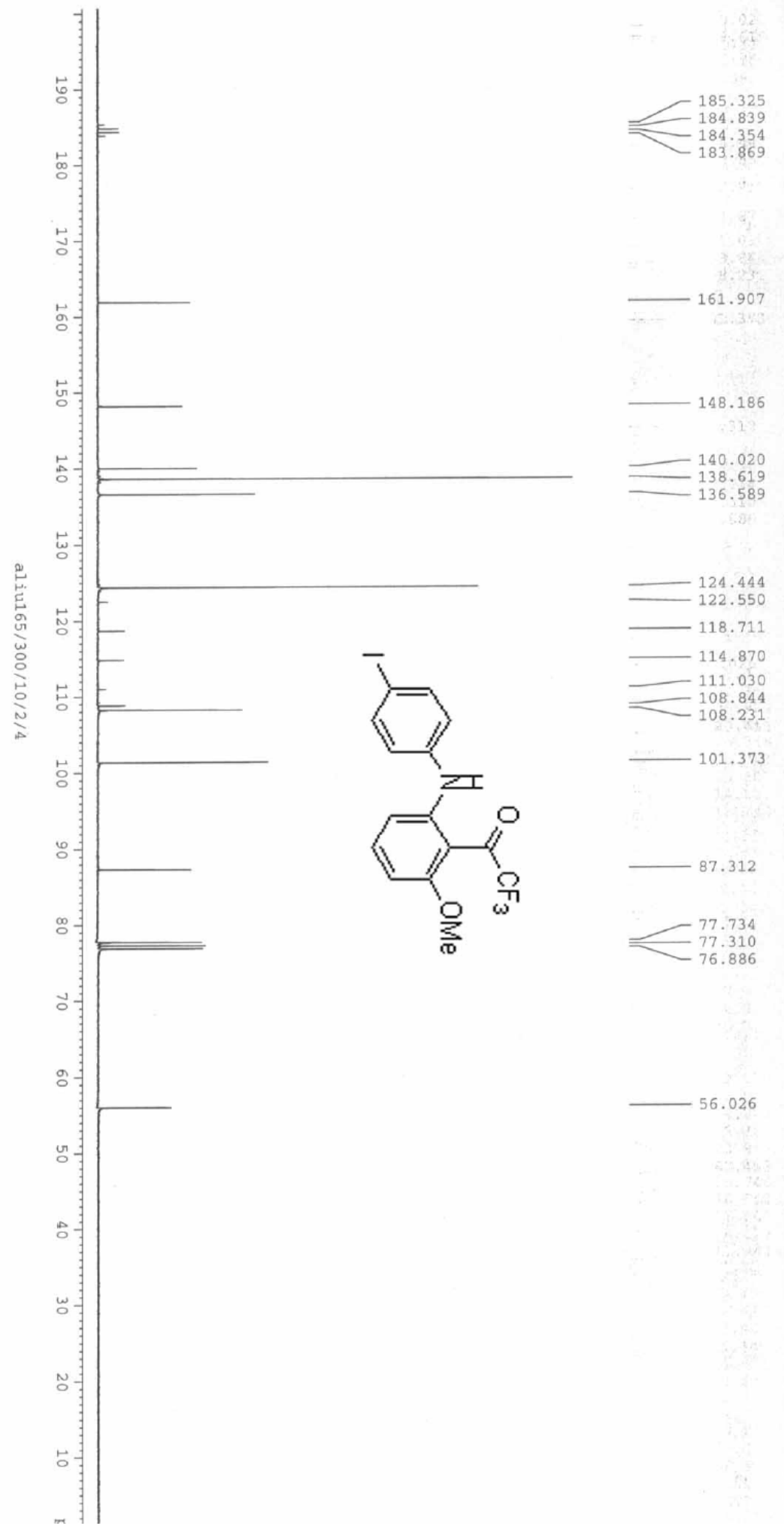



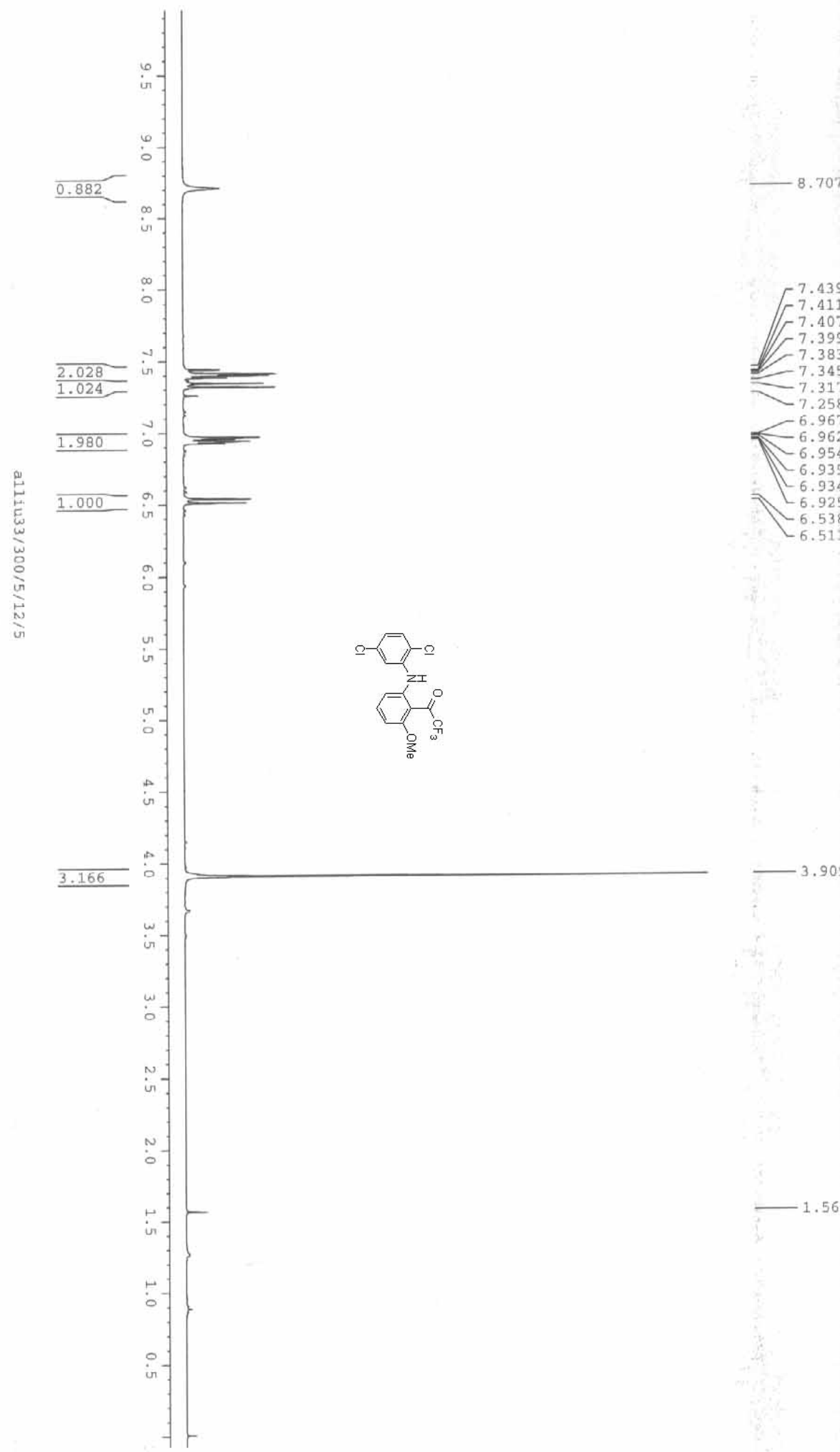

[ 7.439

${ }^{7.411}$

7.399

$-7.383$

$-7.317$

$\begin{array}{r}7.258 \\ -6.967 \\ \hline\end{array}$

$-6.962$

$-6.954$

$-6.934$

$-6.538$

$-6.511$

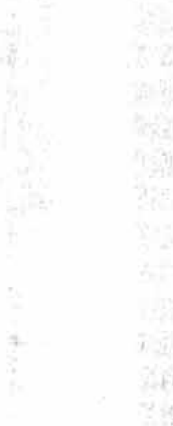

$-3.909$

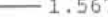




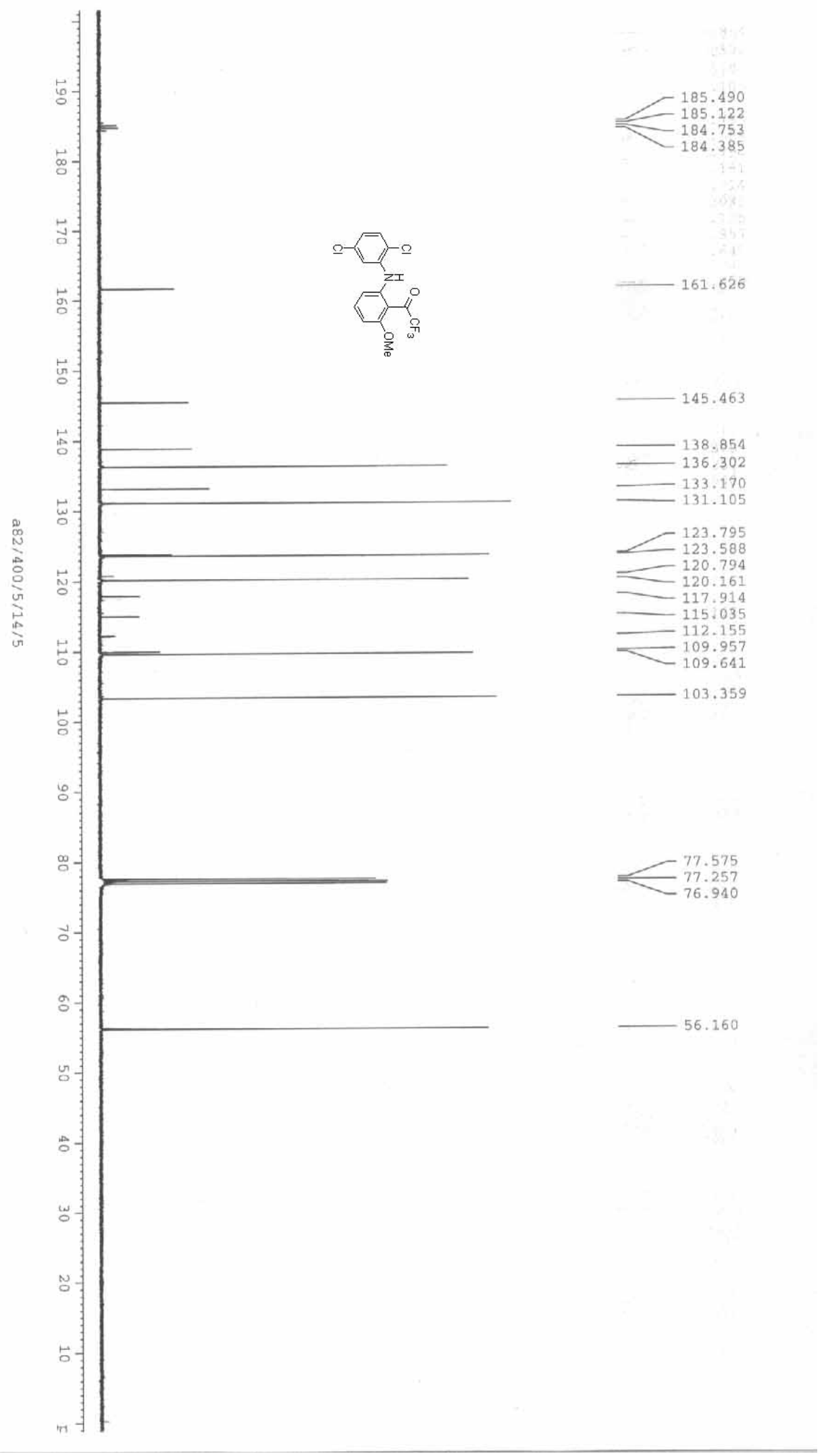




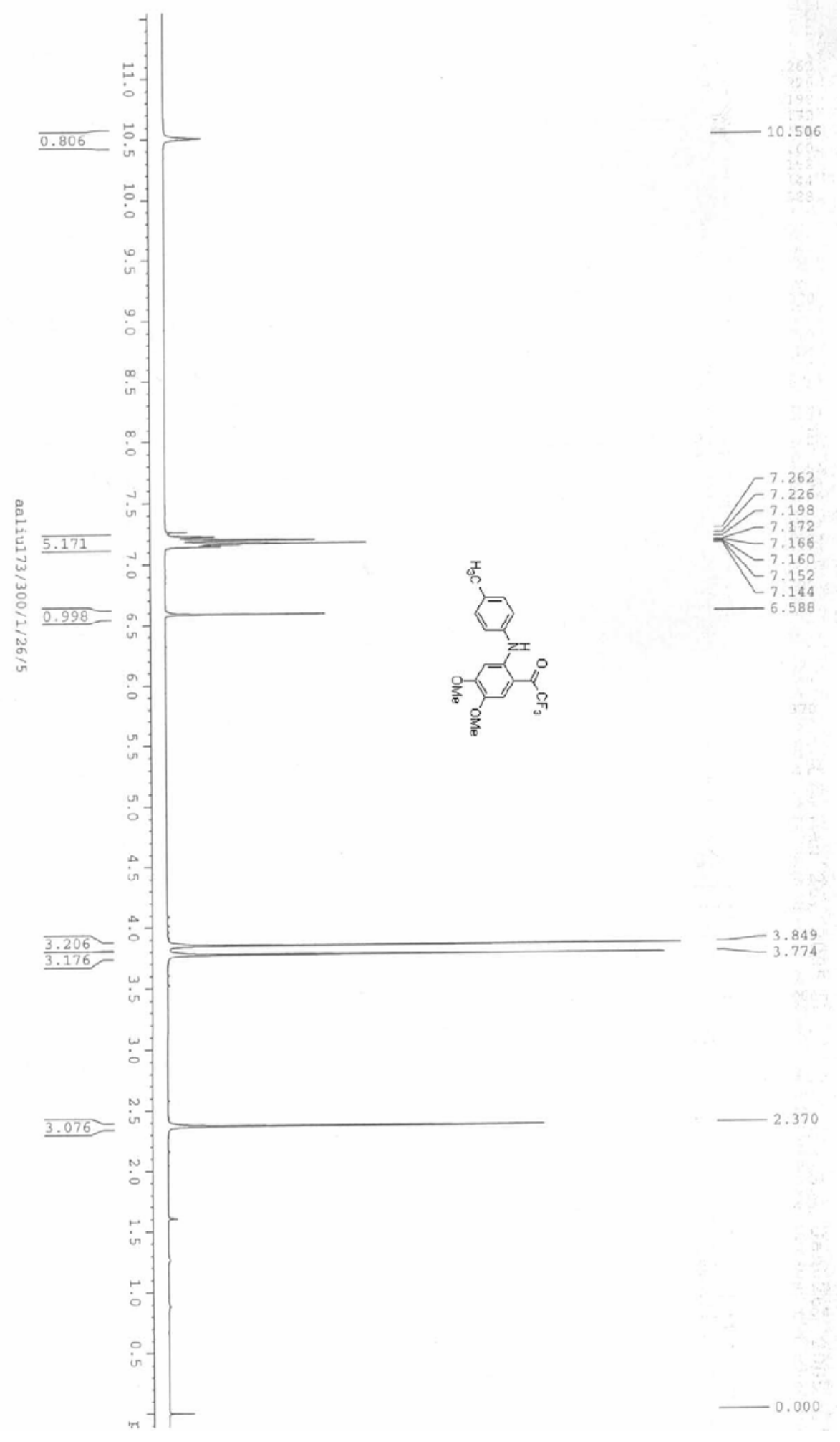




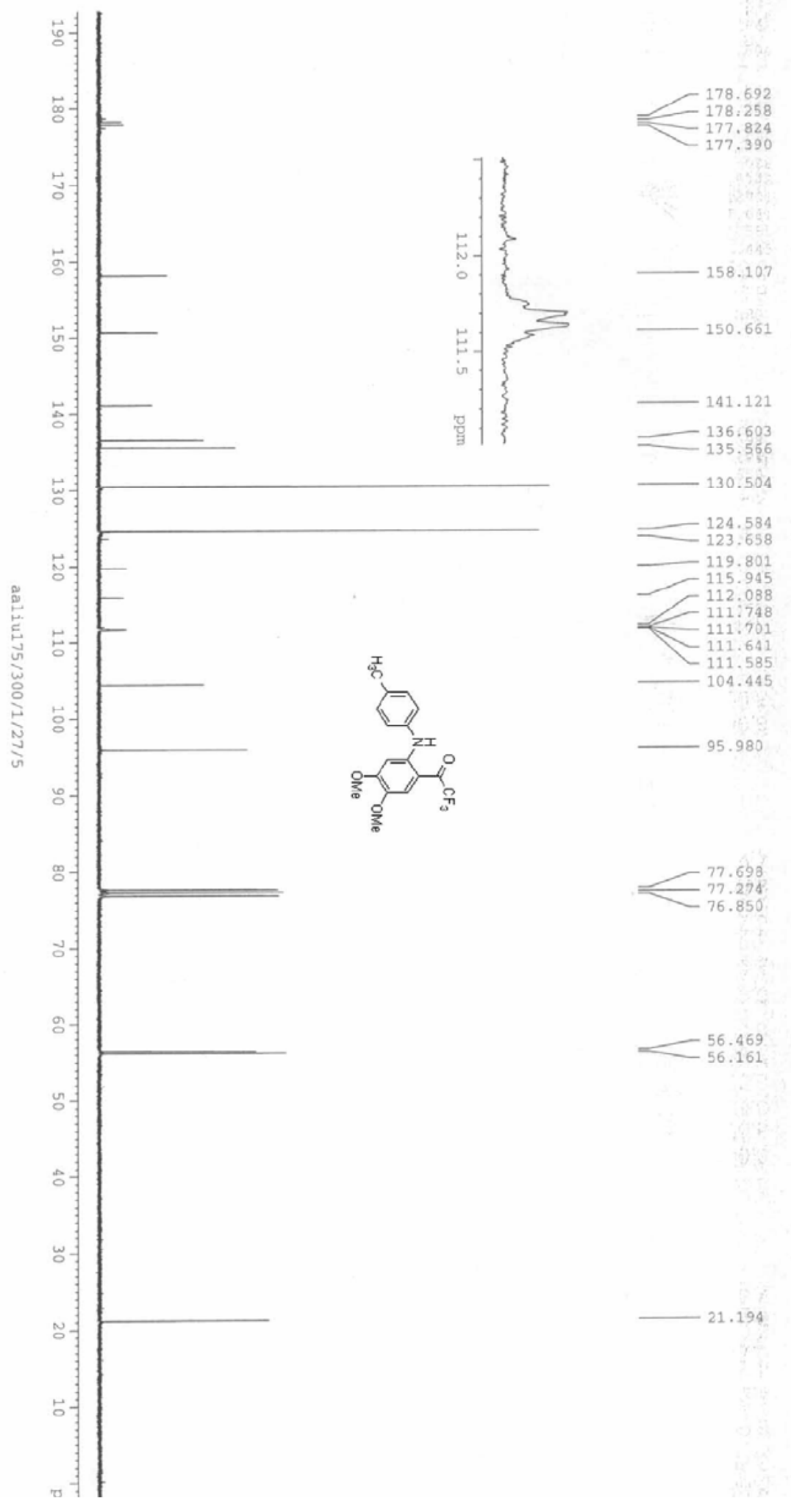




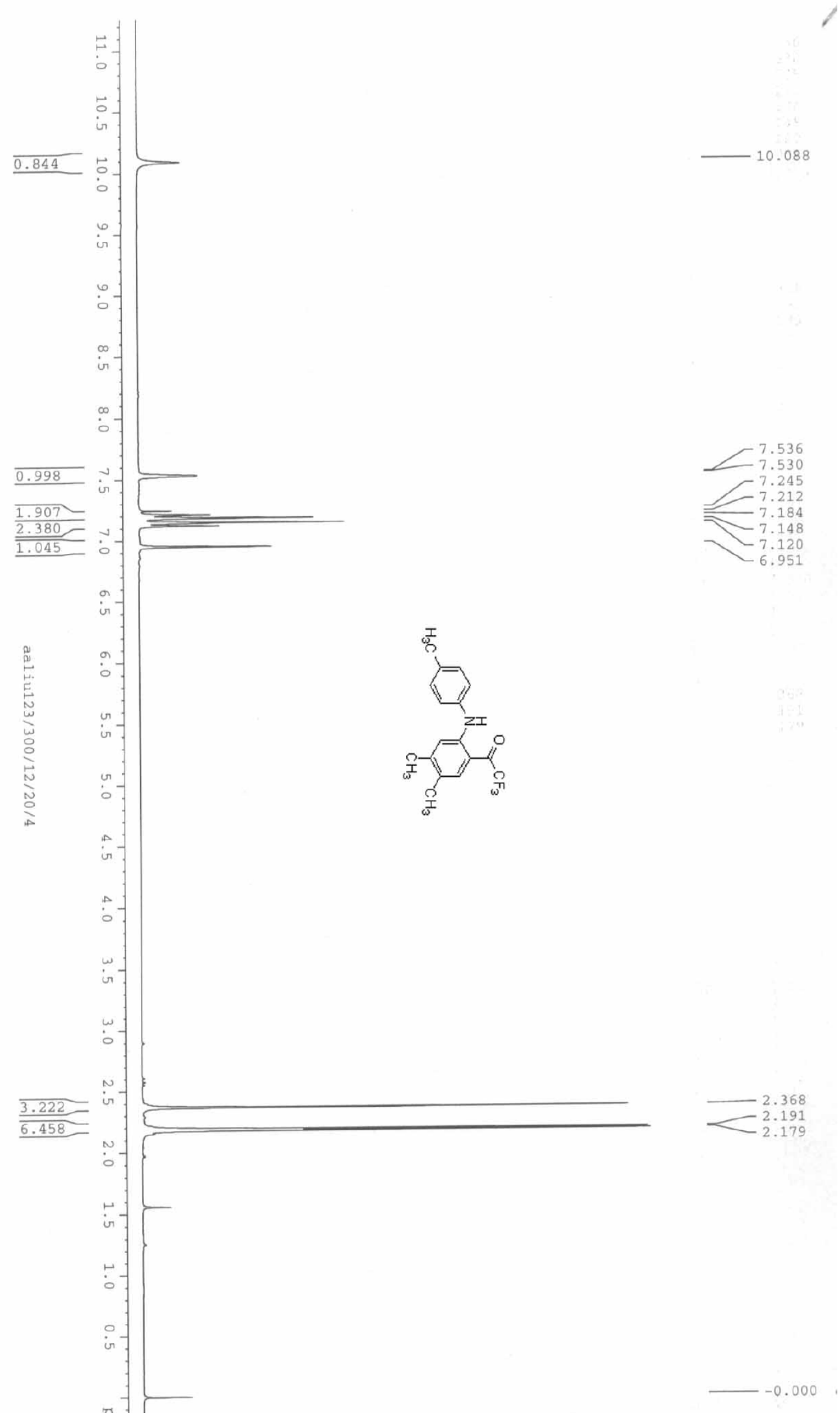



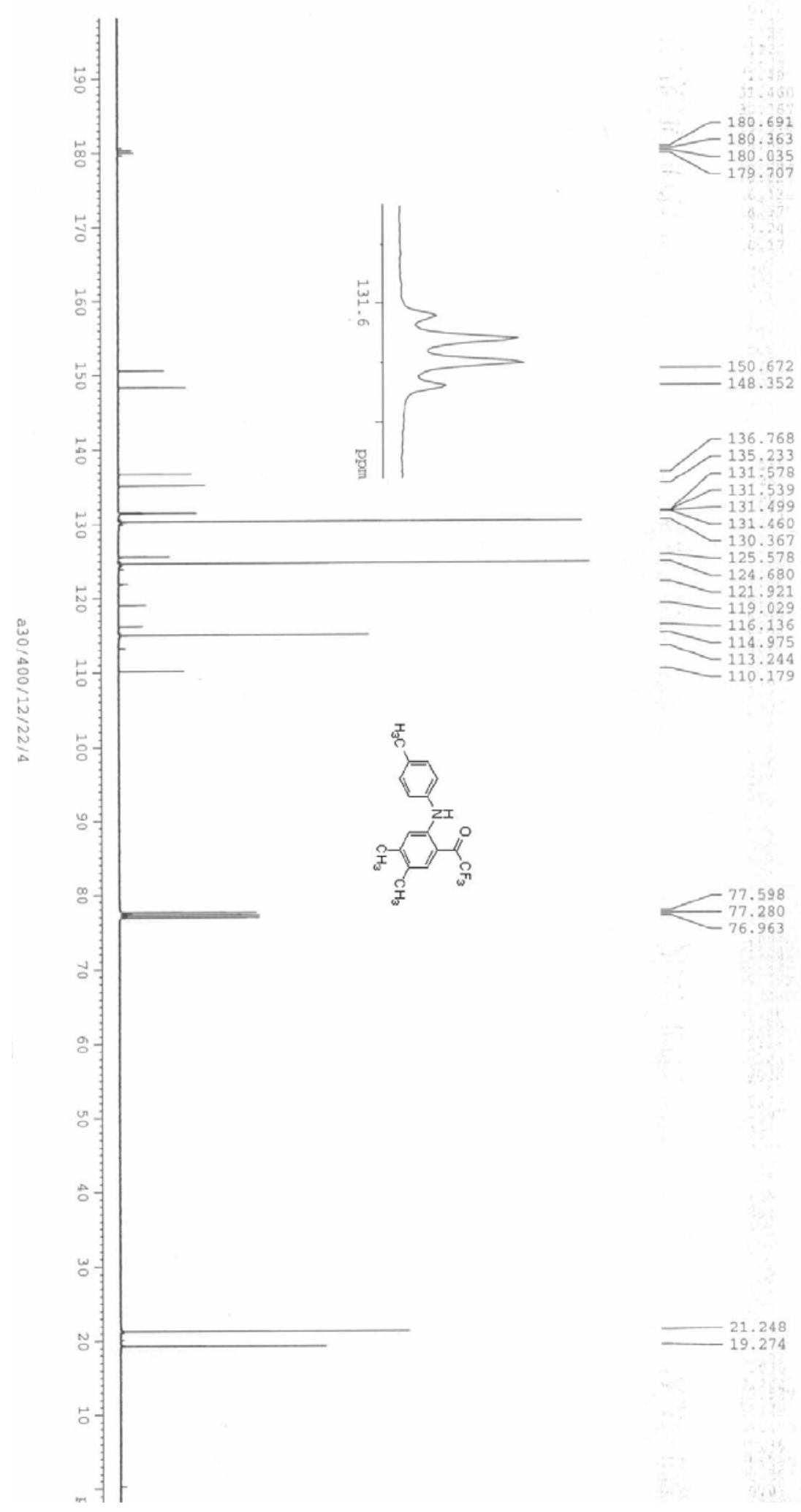


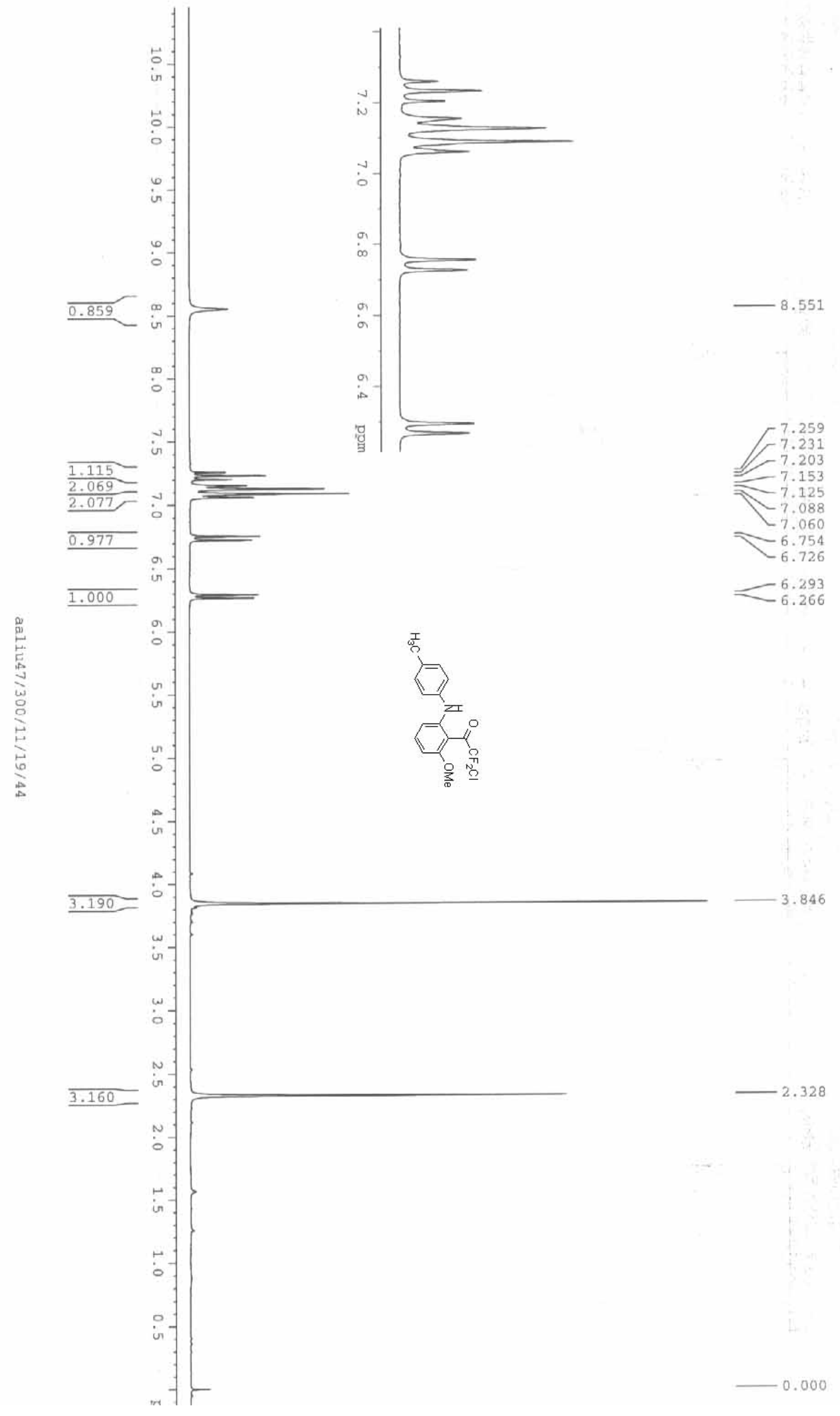




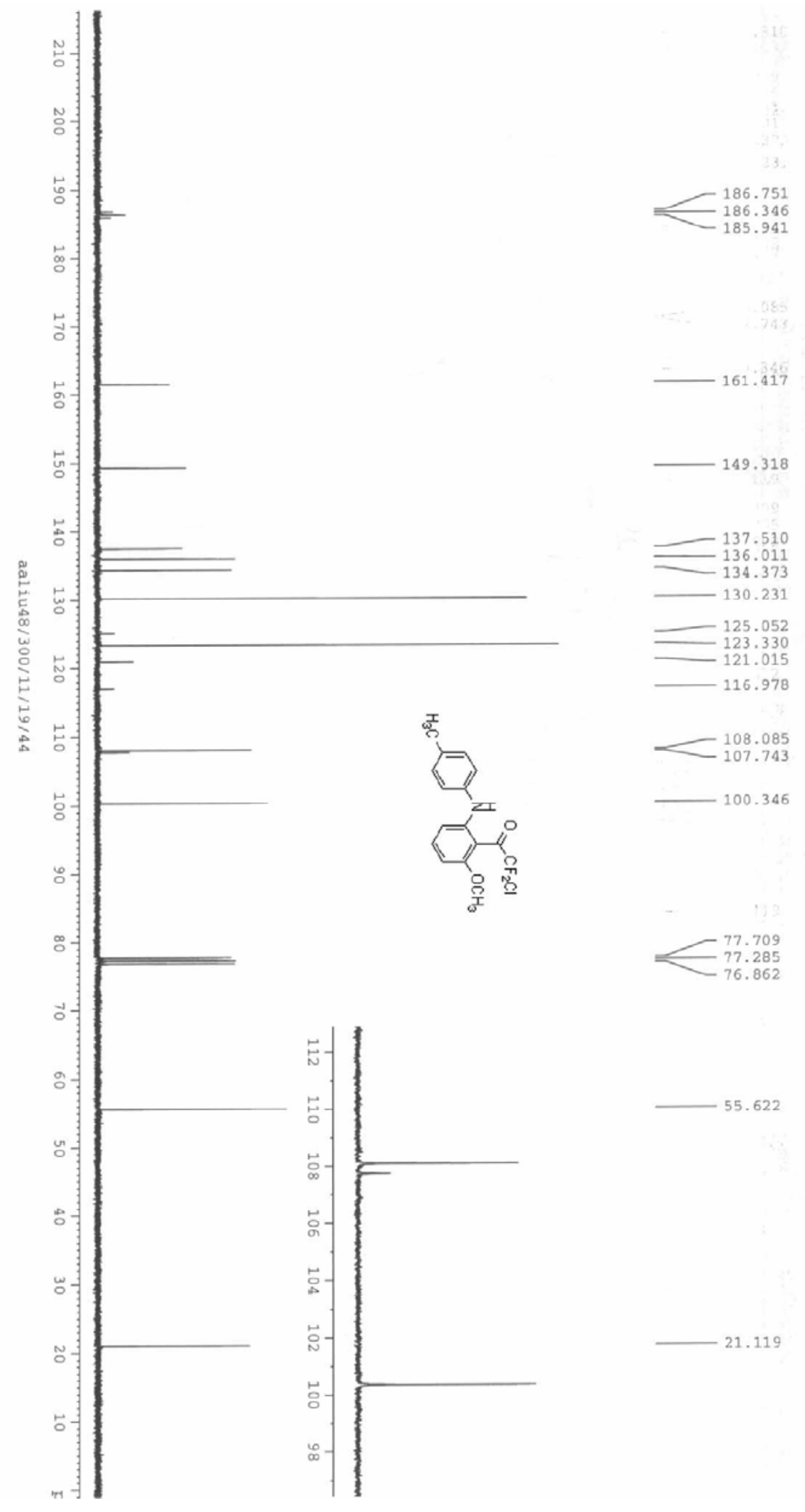



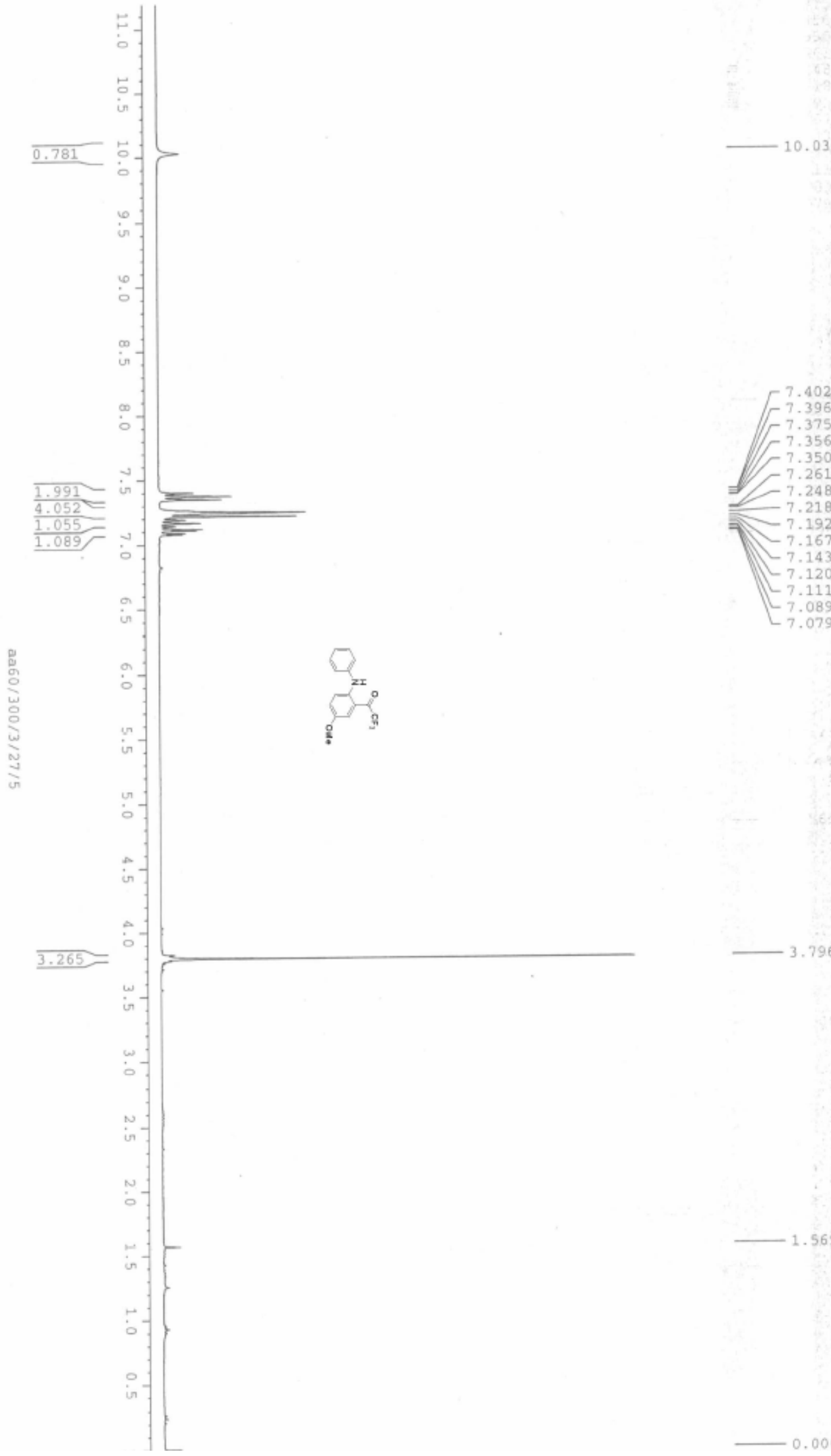

$-7.375$

$F^{7.356}$

$F^{7.350}$

$\begin{array}{r}7.256 \\ -7.248 \\ \hline\end{array}$

-7.218
-7.192

$7-7.267$

$-7.143$

- 7.111

$-7.089$

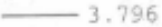

3.796

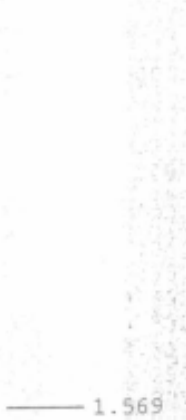

0.000 

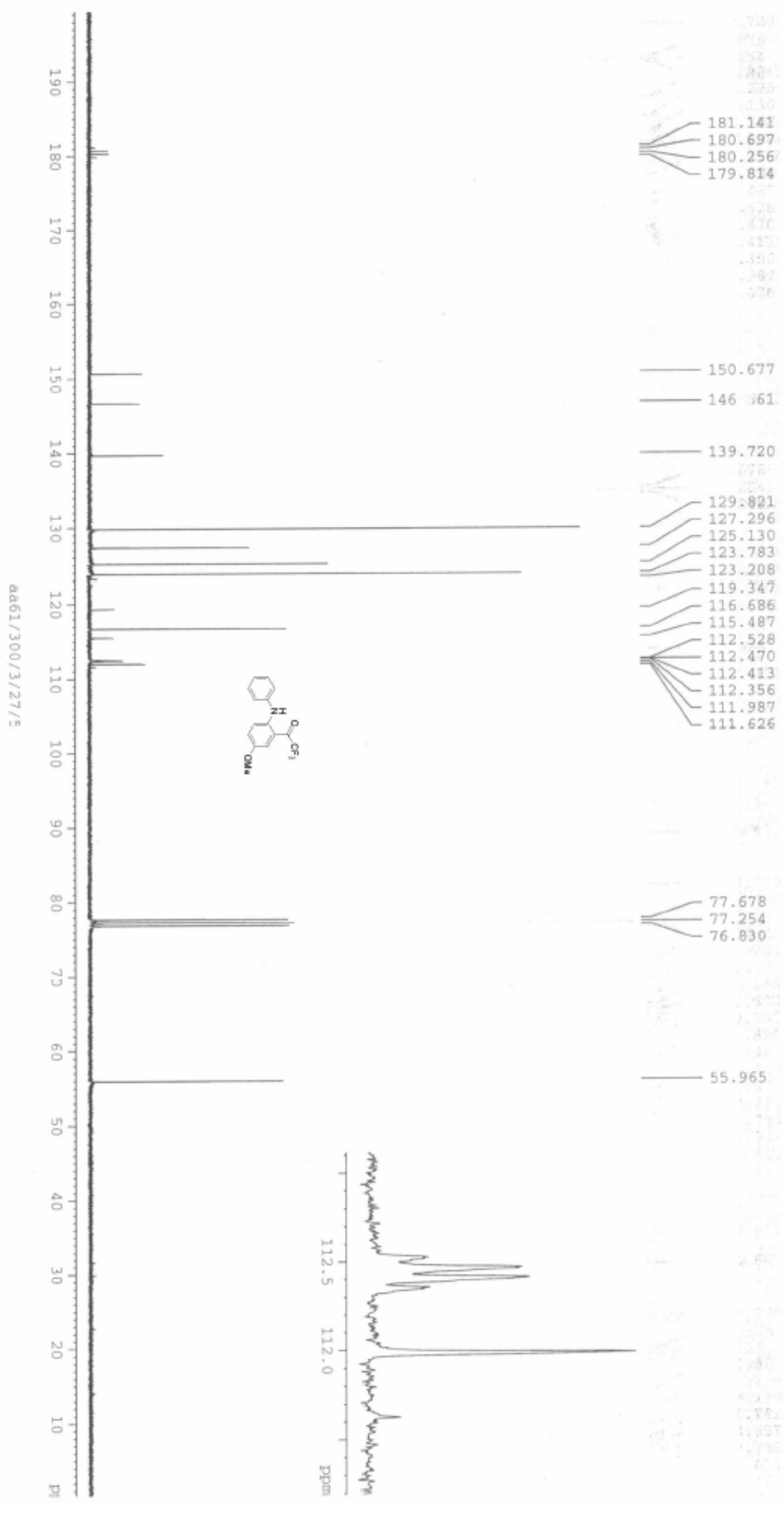

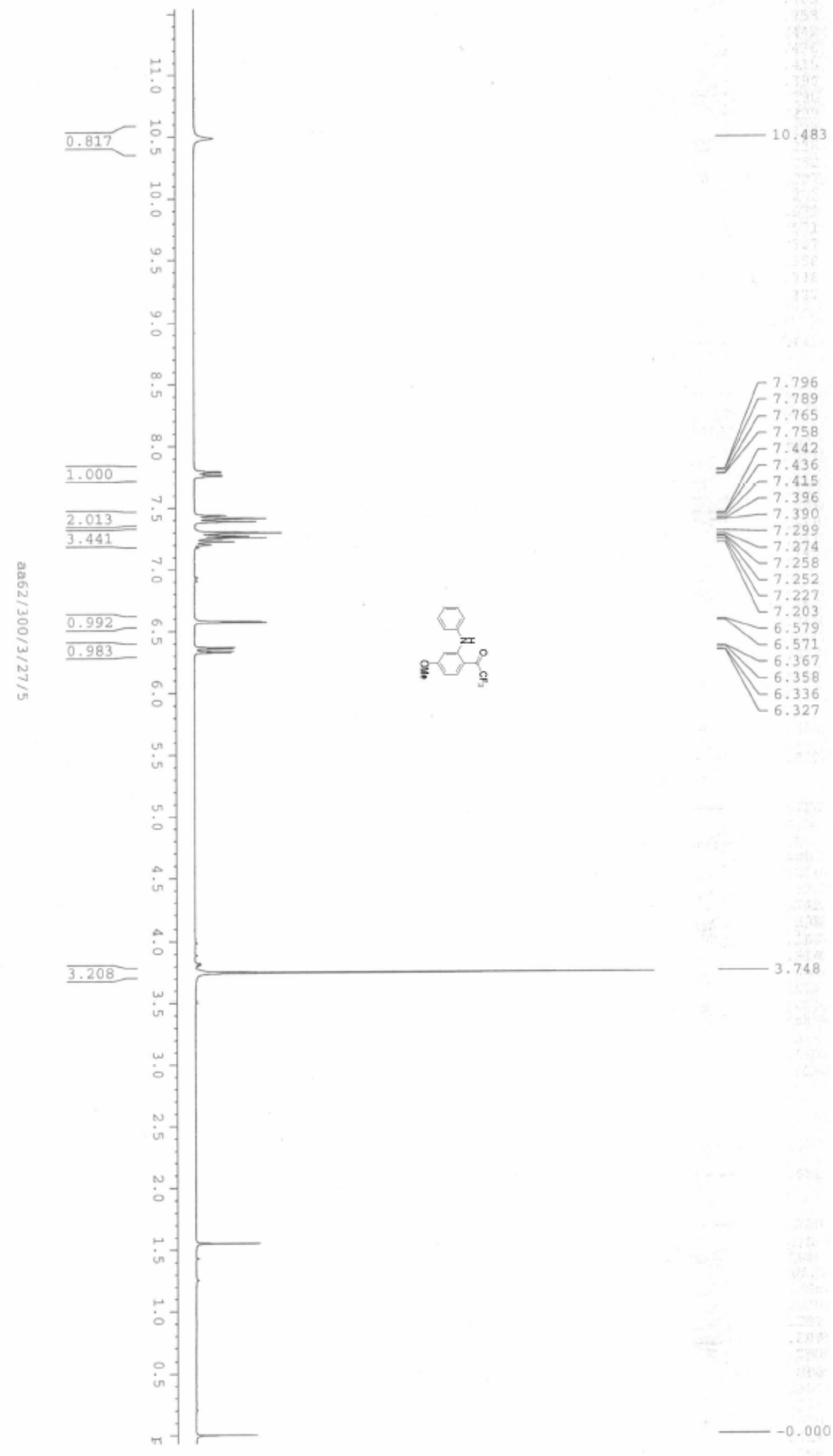


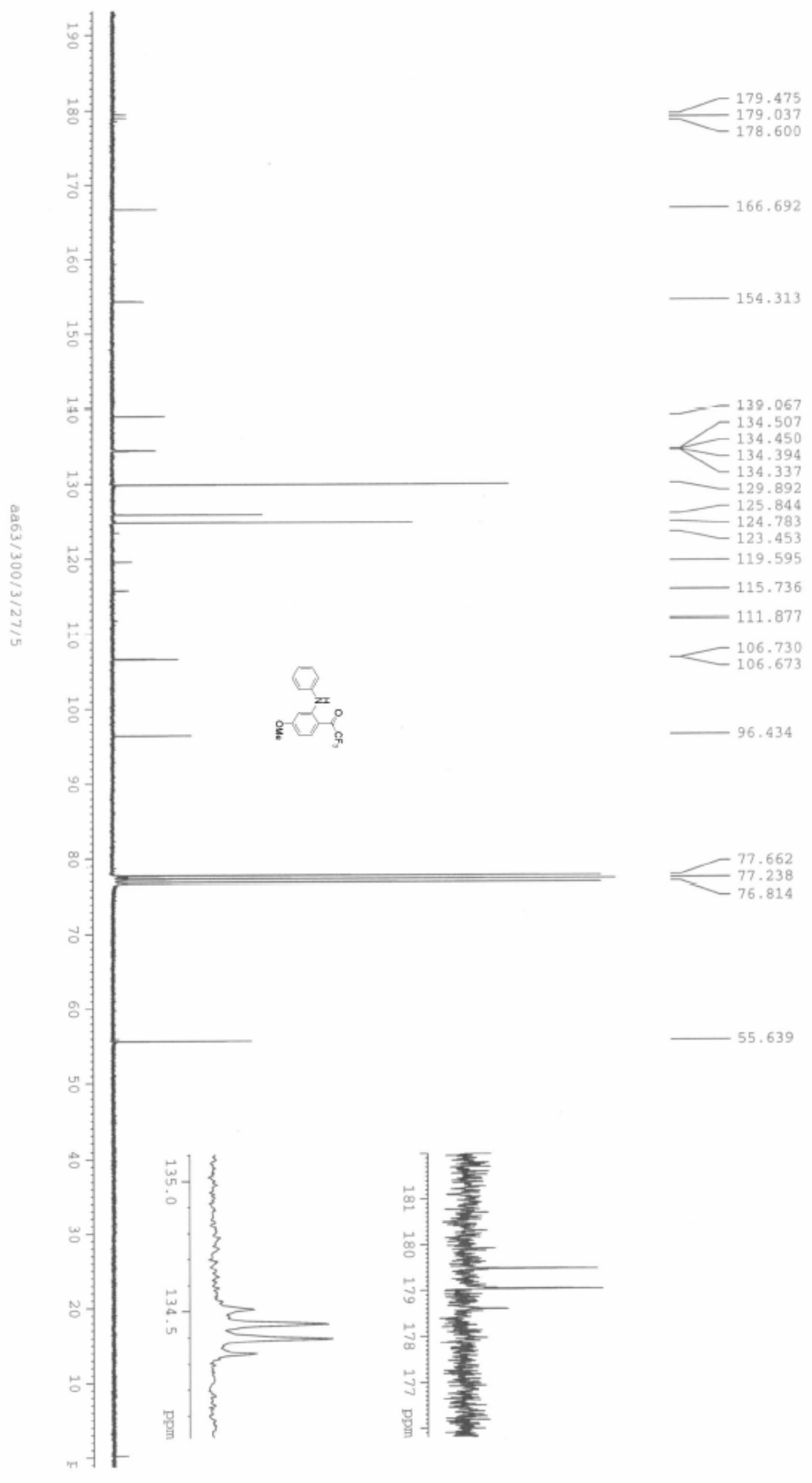




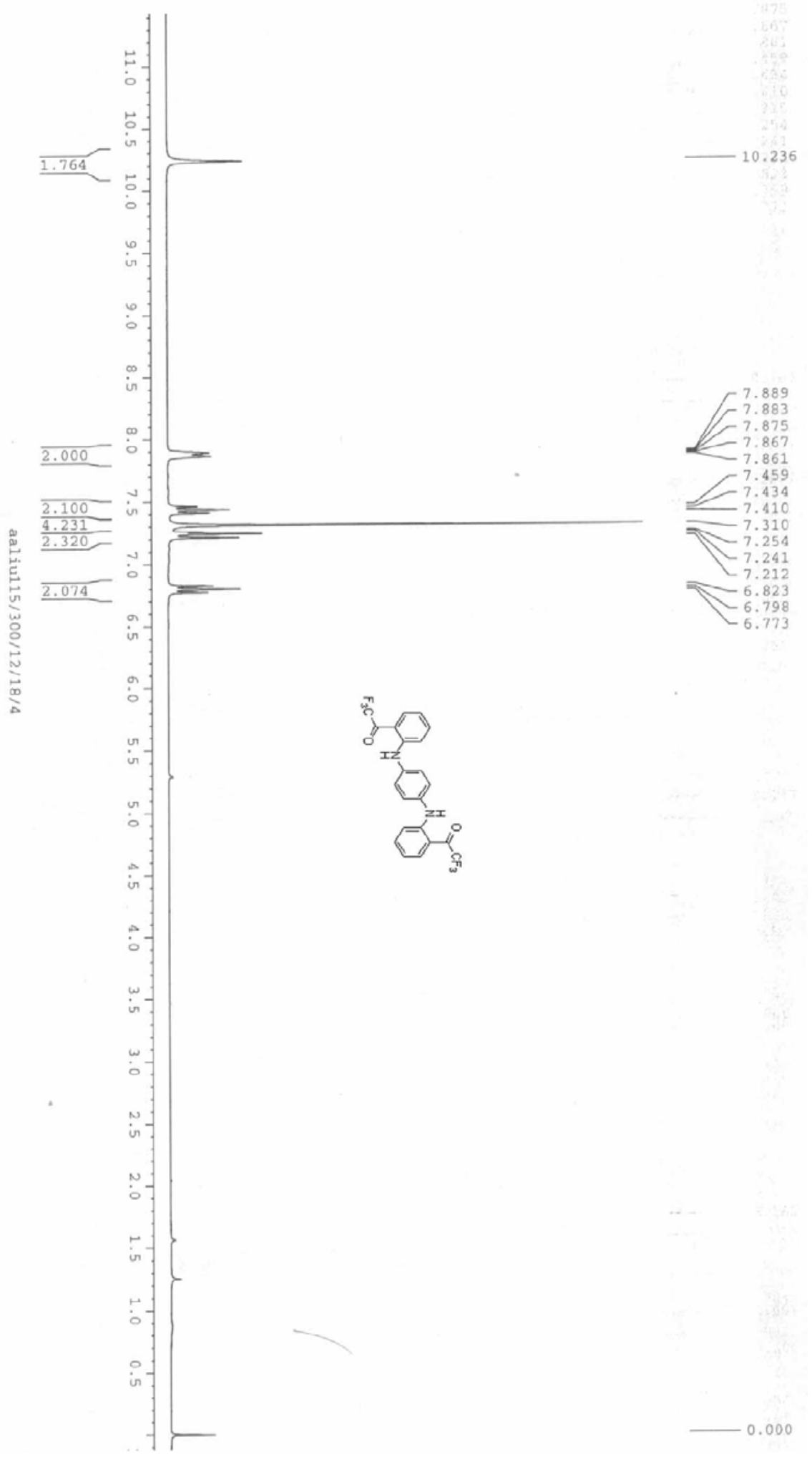




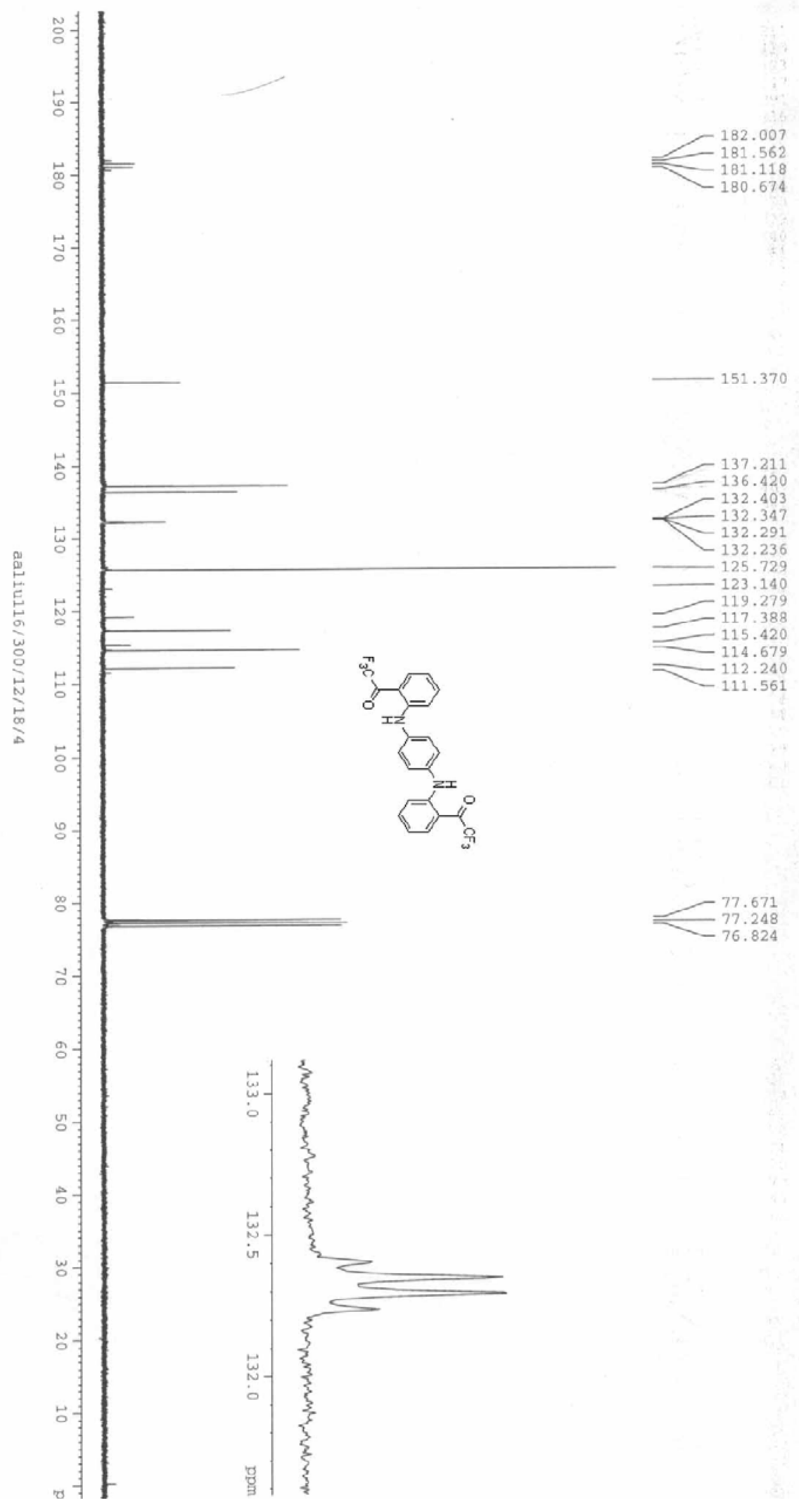



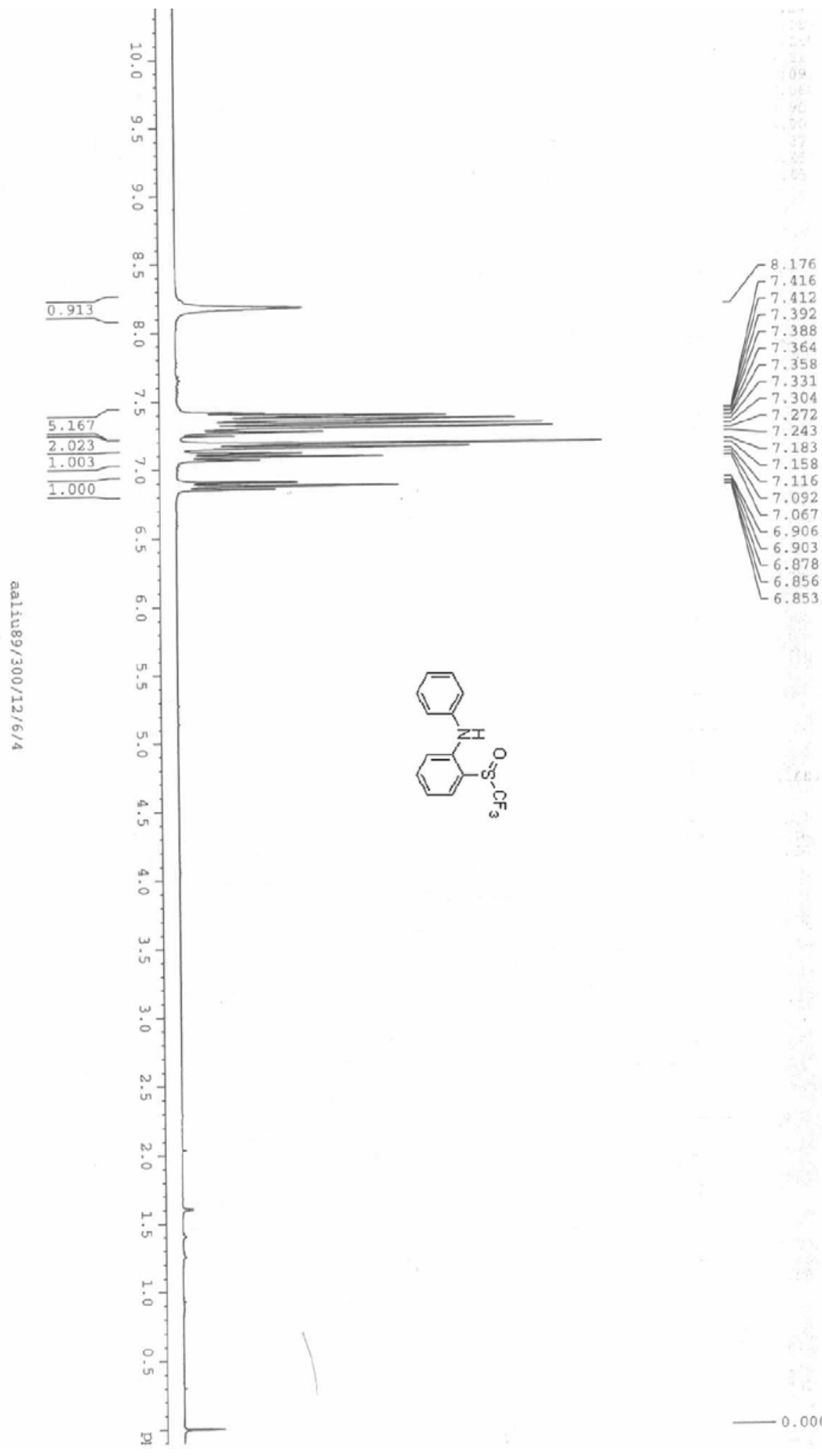


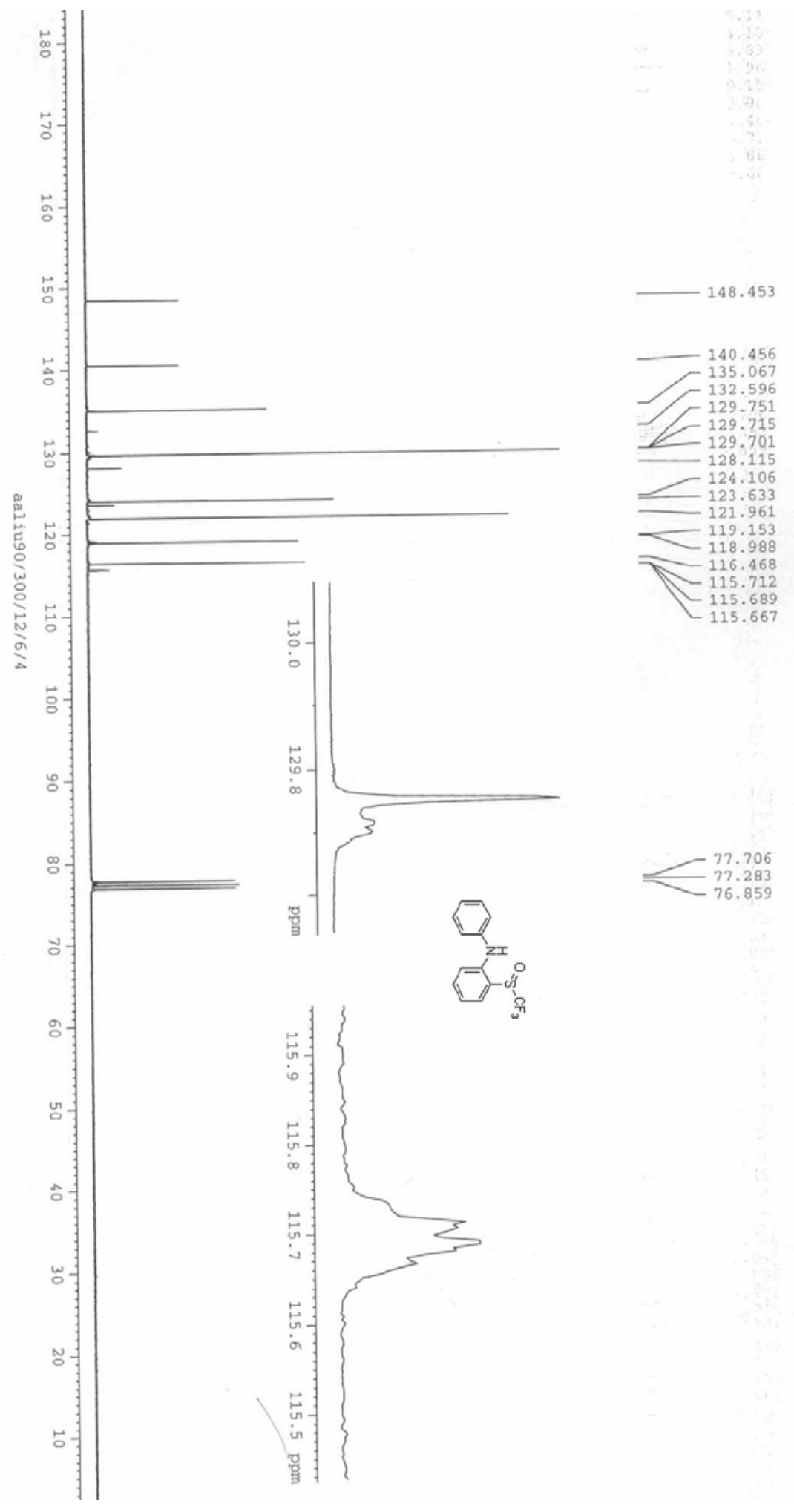



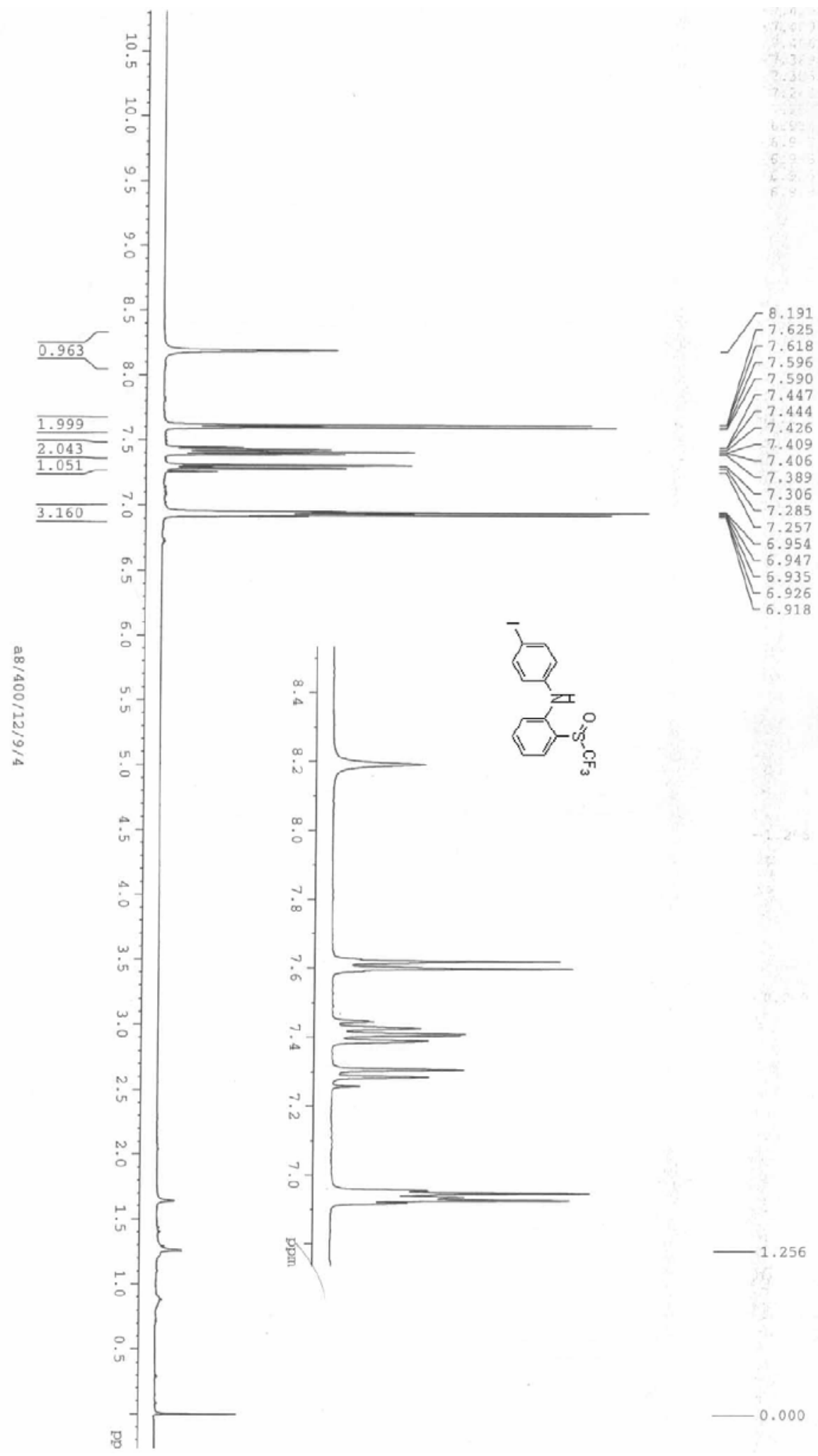


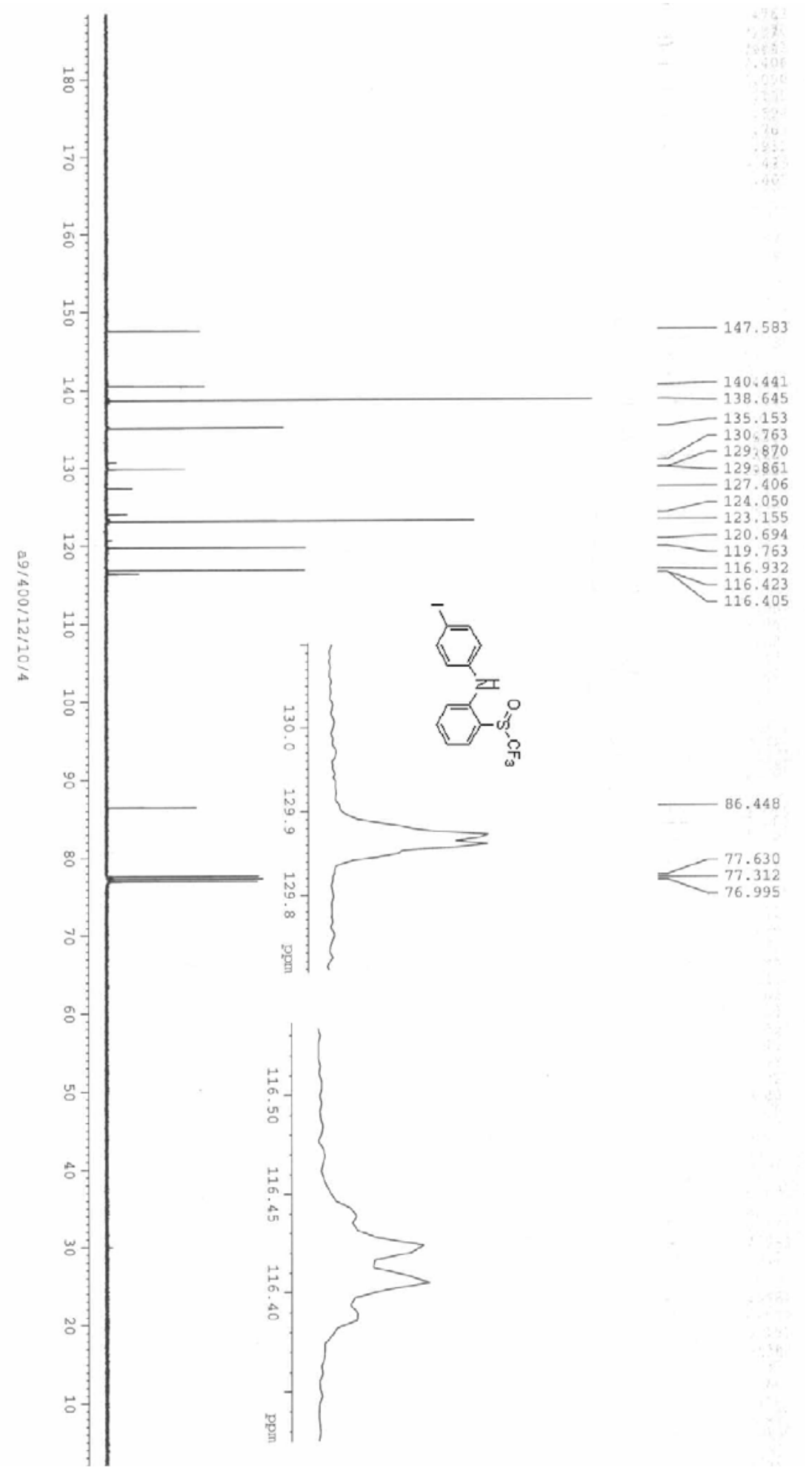




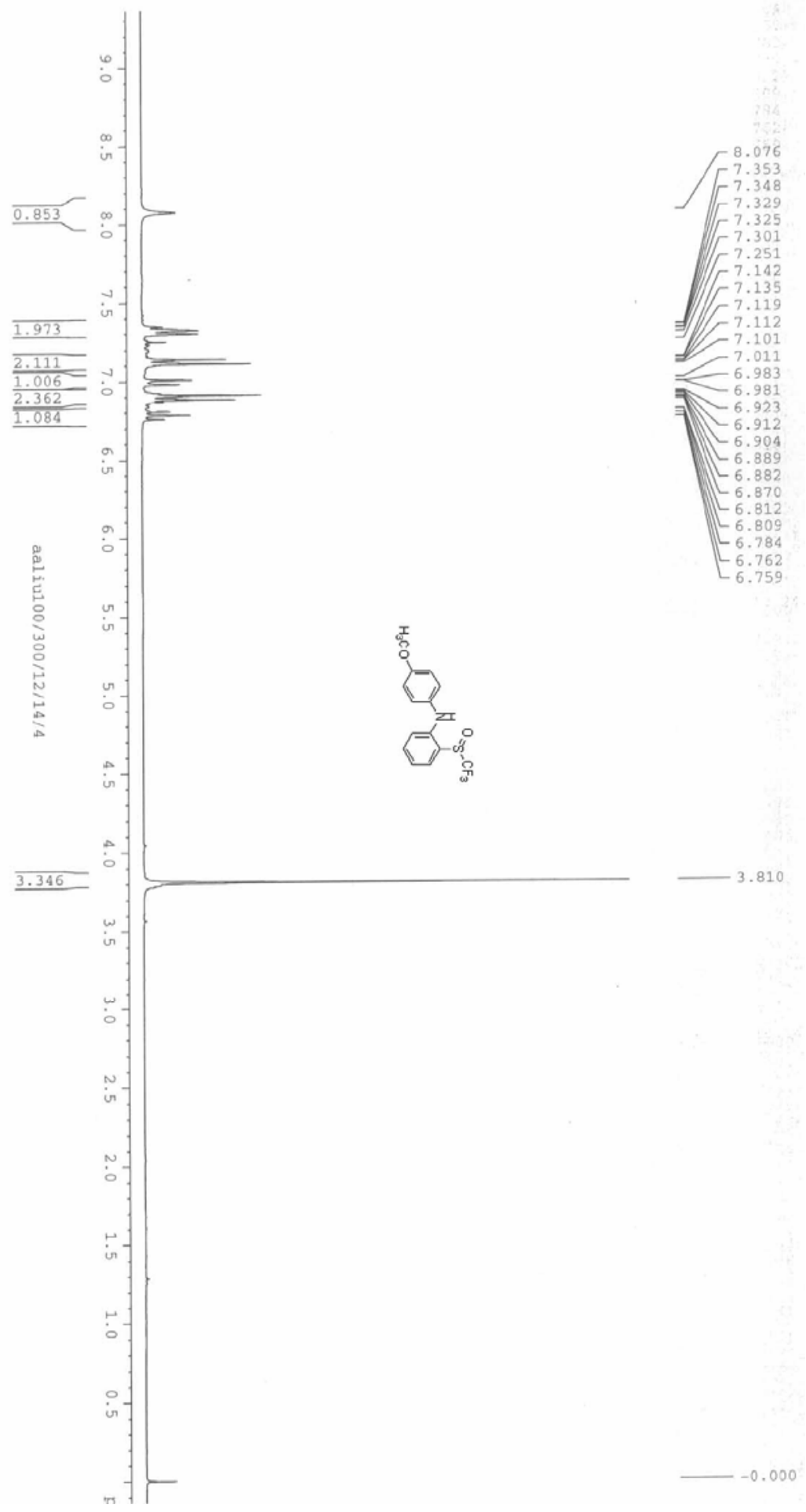




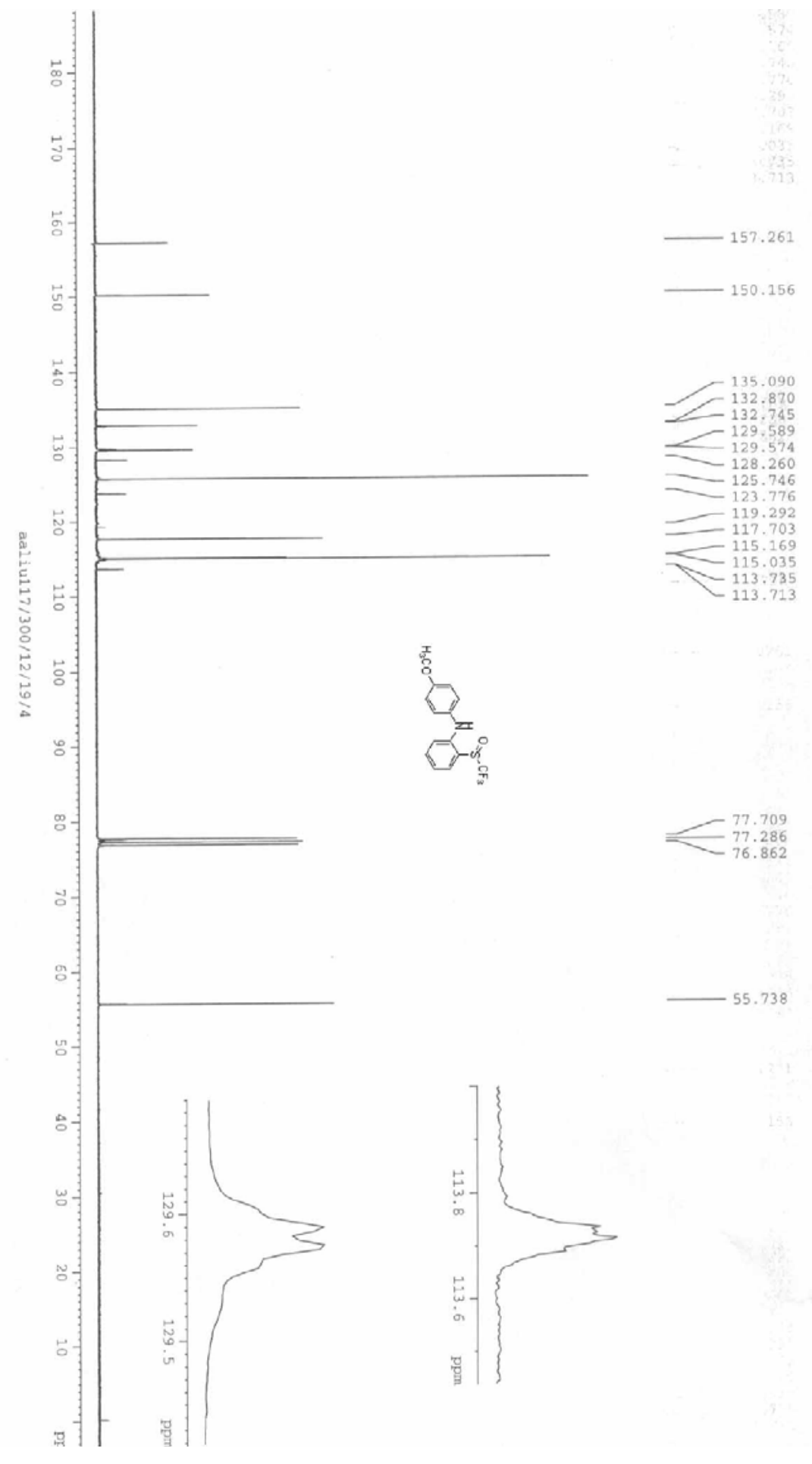




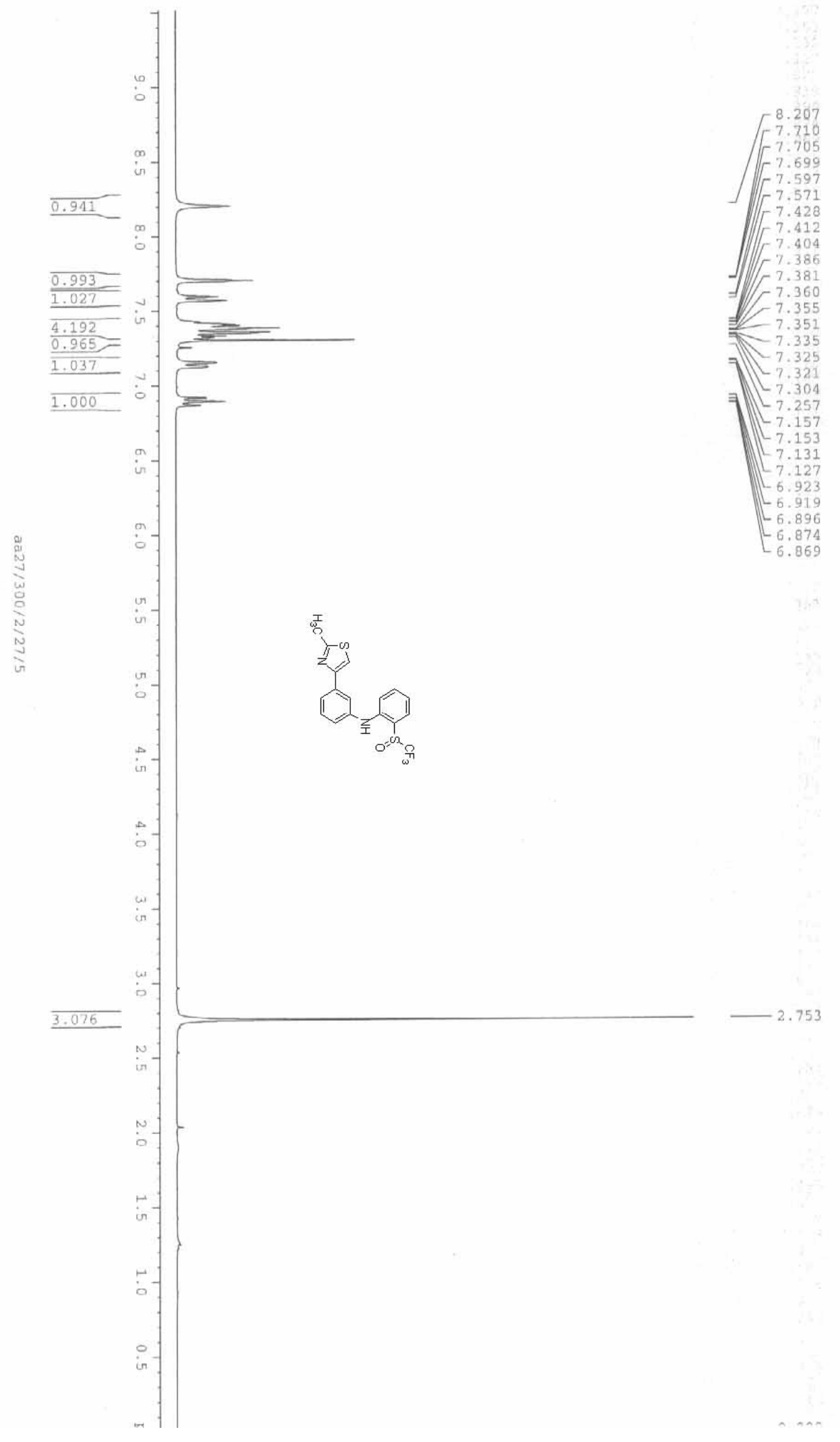



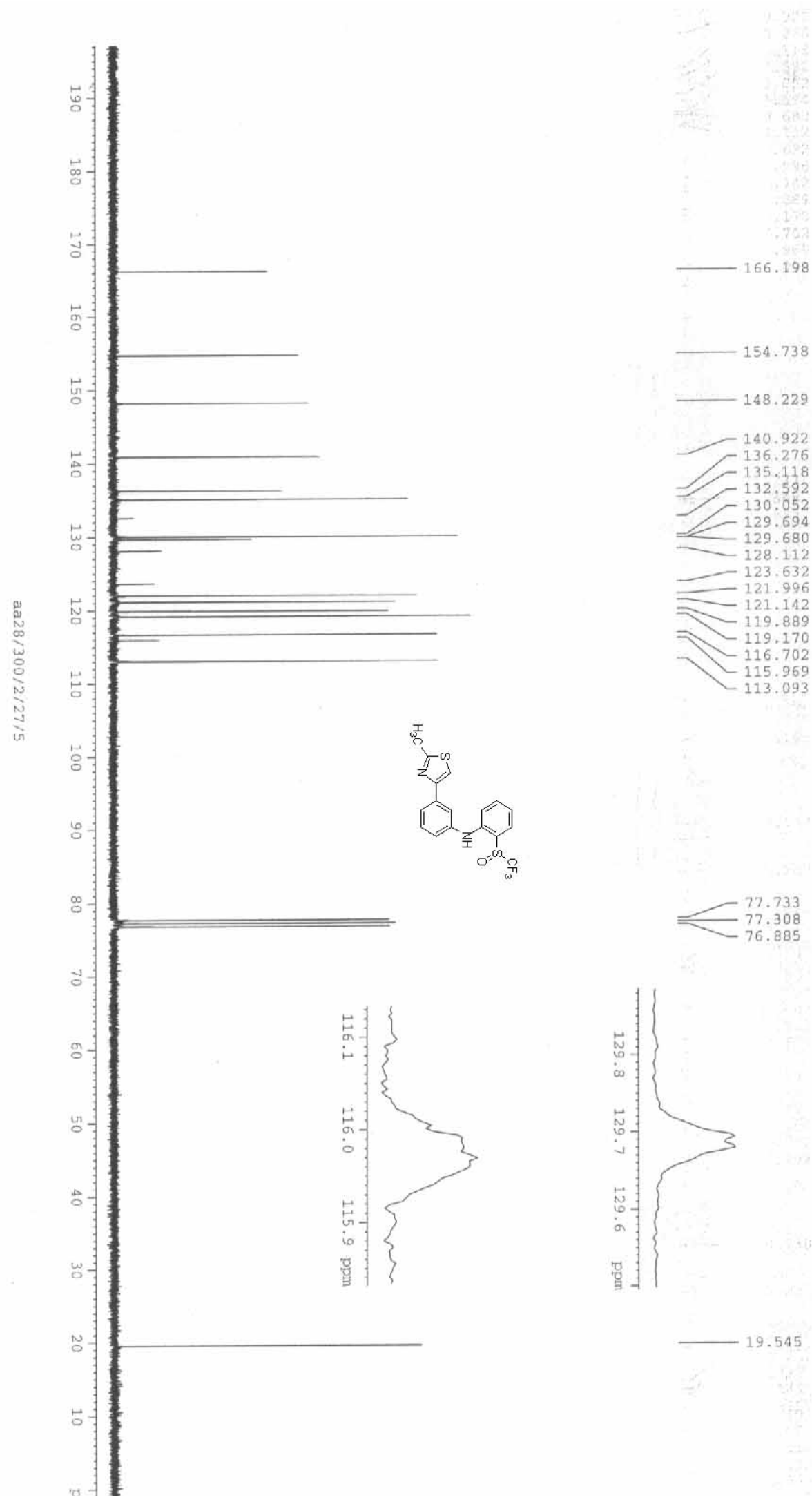

$-136.276$

135.118
-132.592

130.052

129.694

$-123.632$

- 121.996

$-119.142$

$\longrightarrow 119.170$

115.969
-113.093

\begin{tabular}{r}
77.733 \\
-77.308 \\
\hline \\
76.885
\end{tabular}

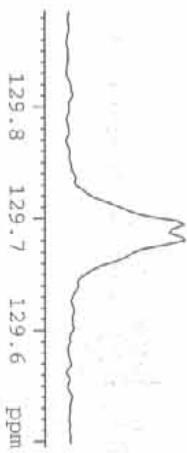



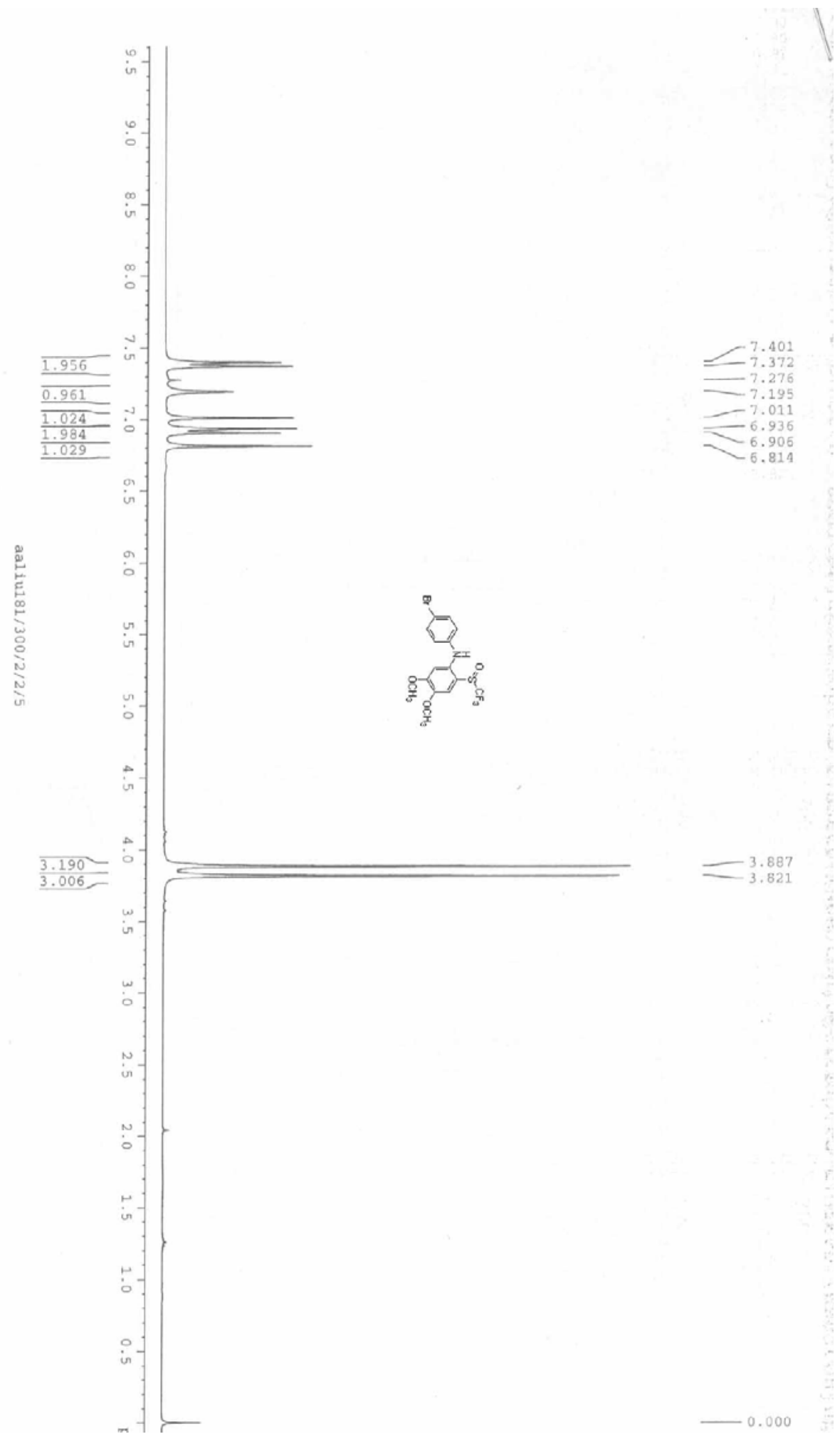


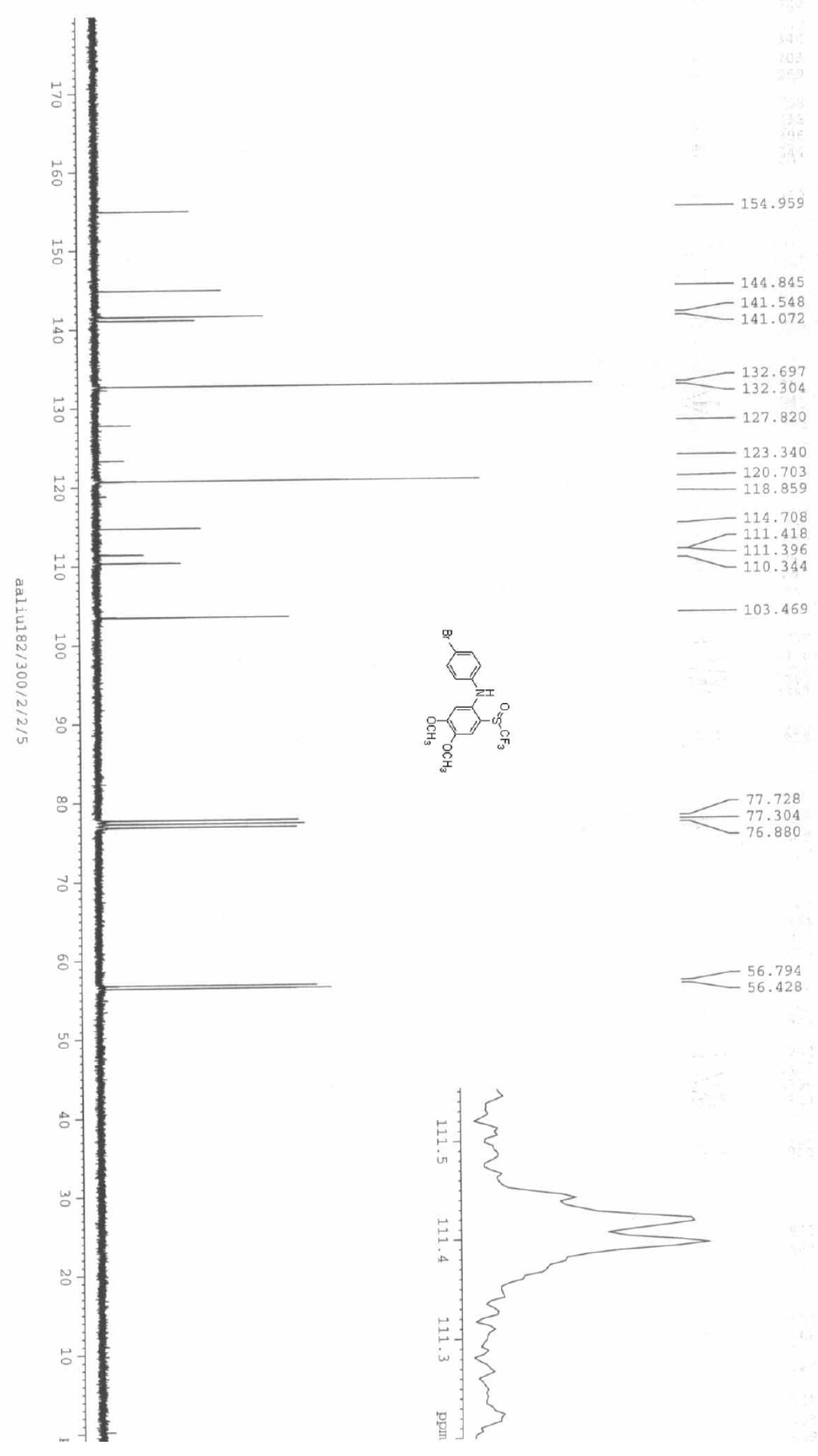



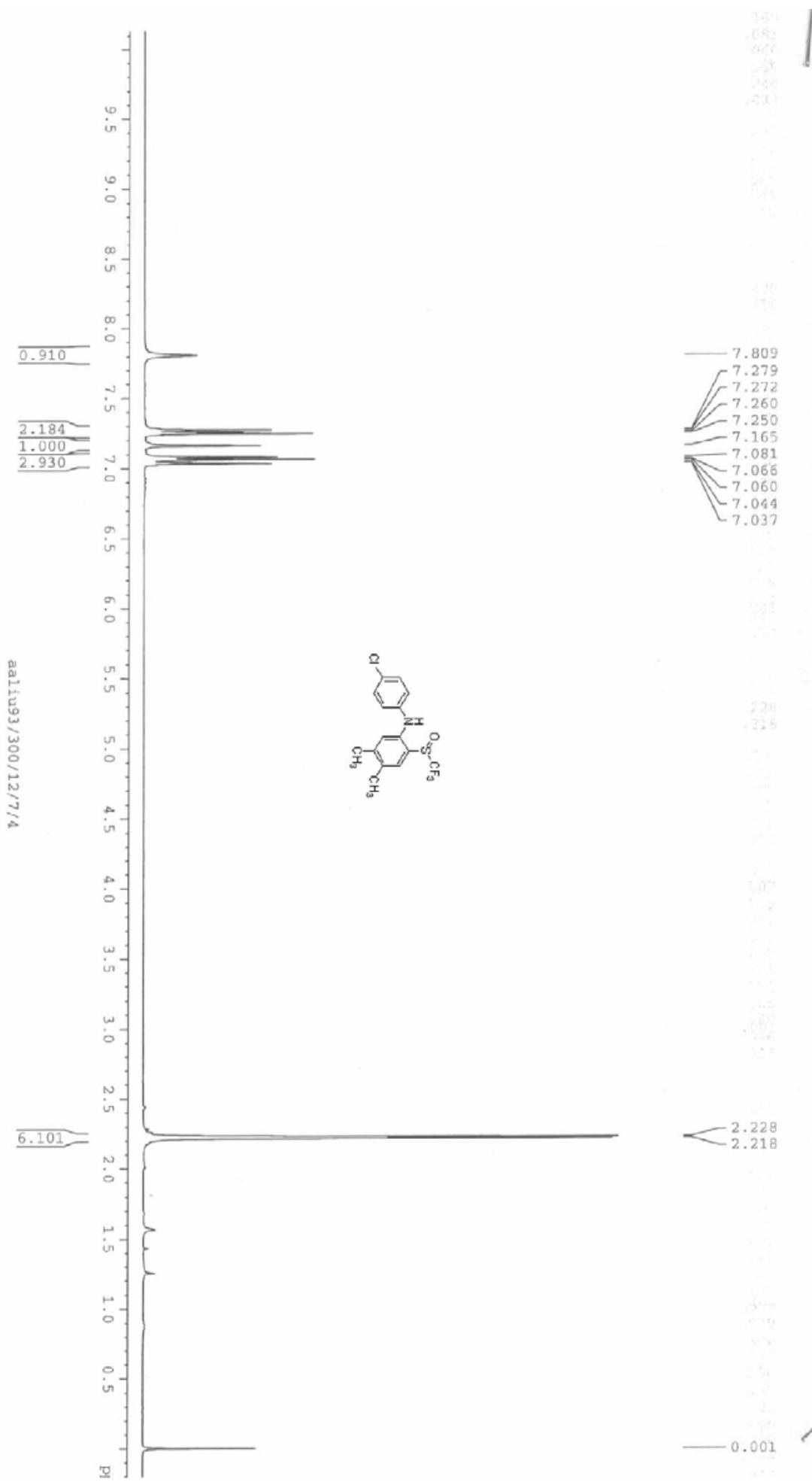

$-0.001$ 


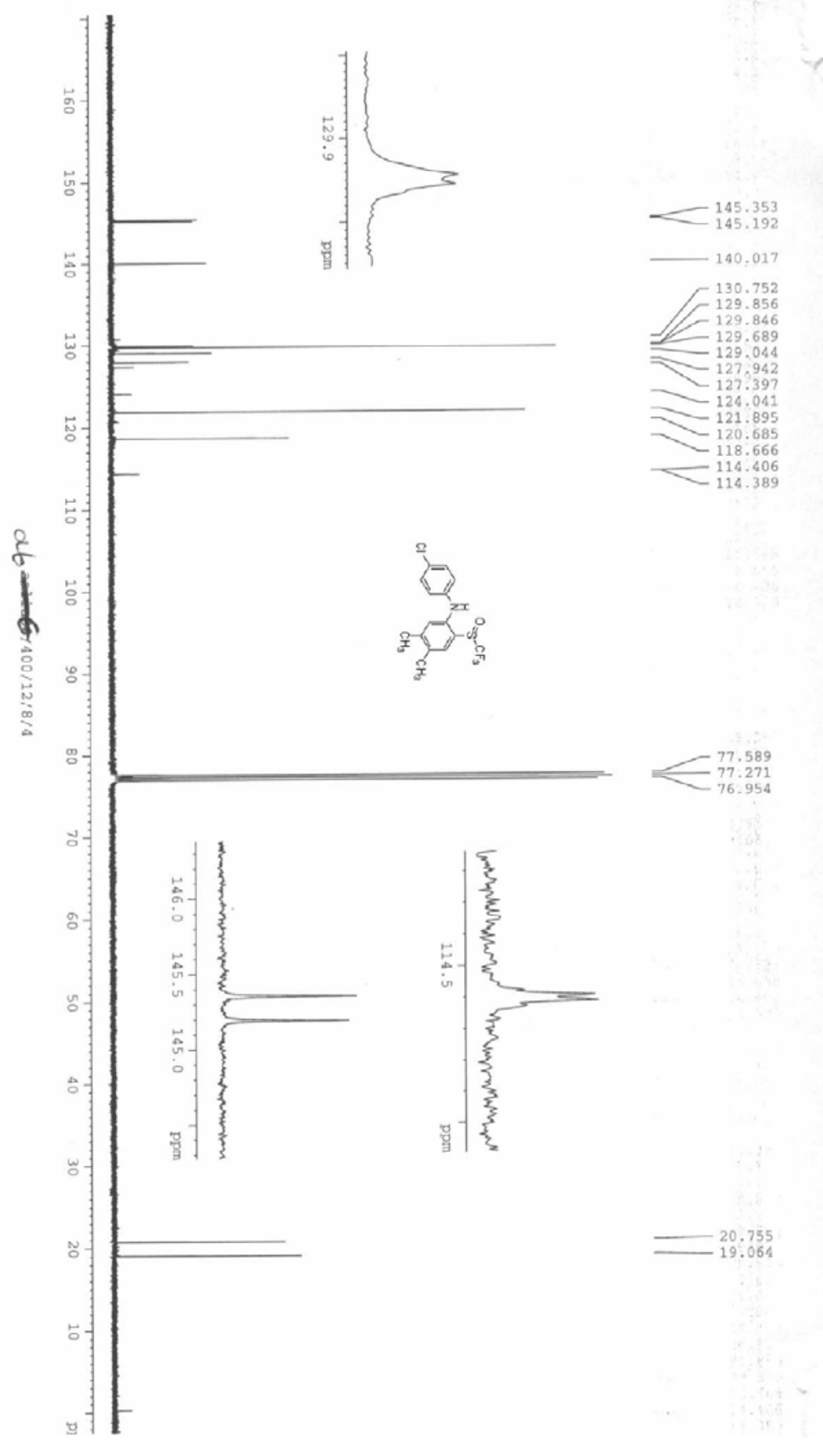



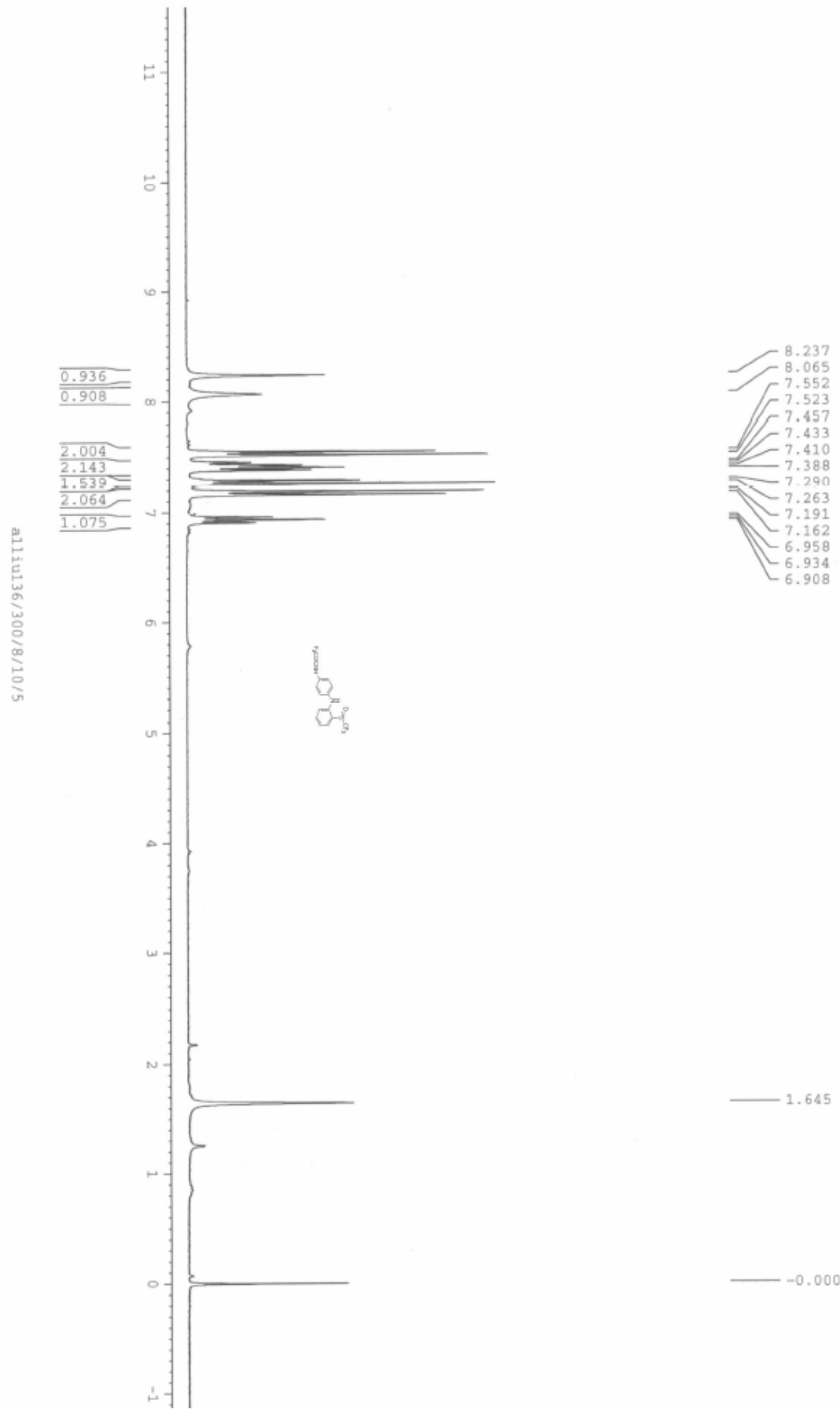


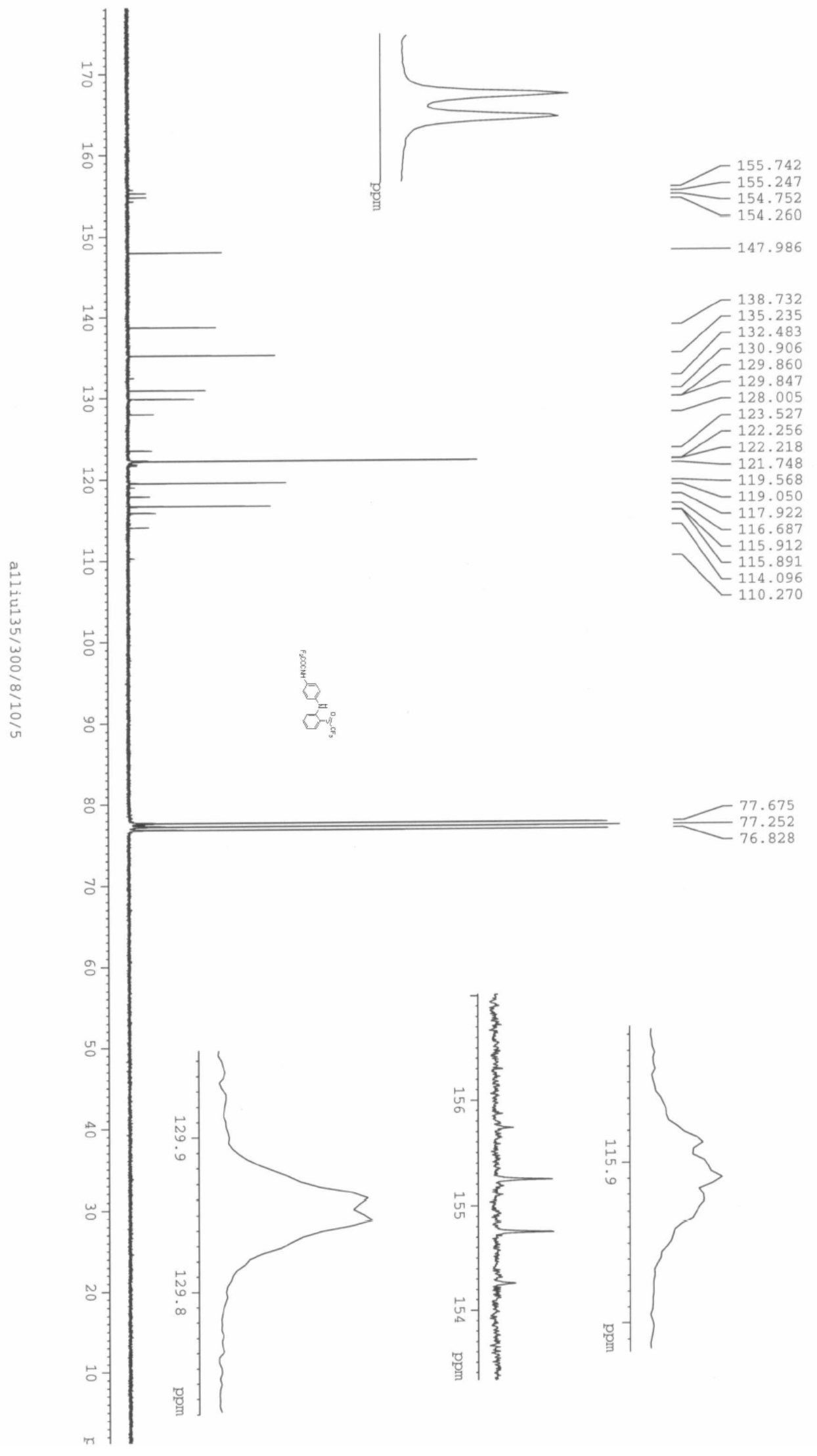




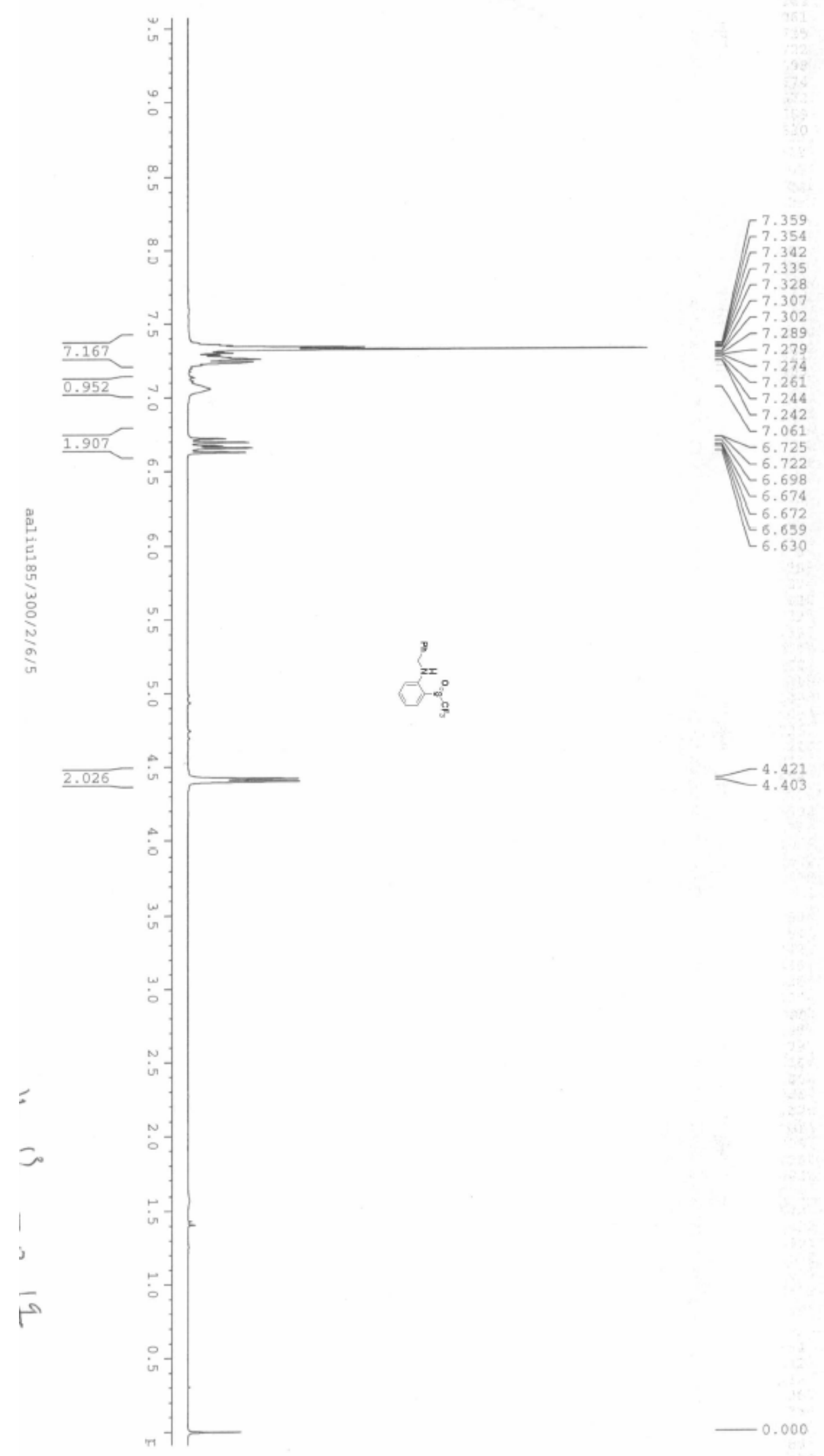




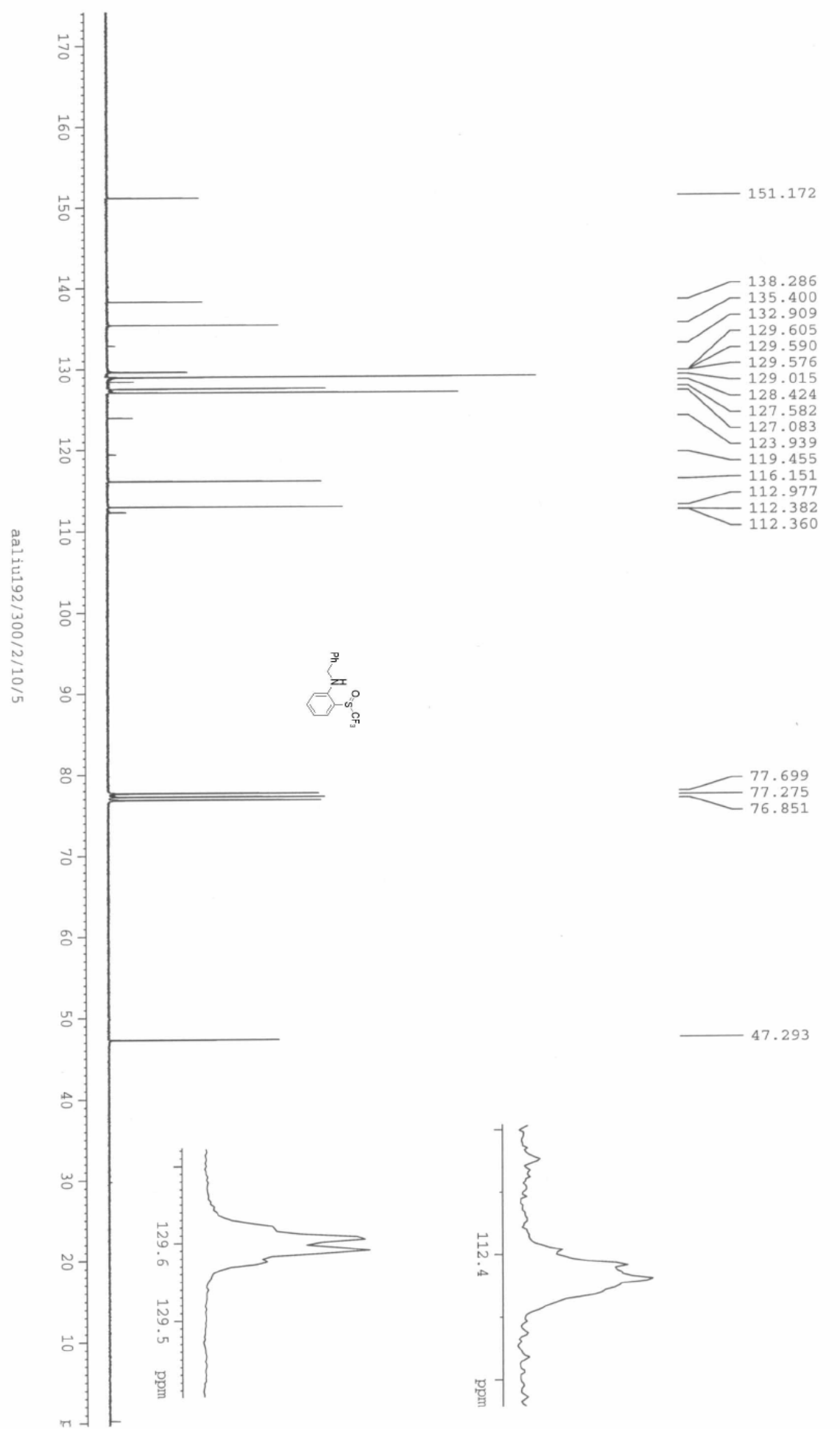

